\title{
Design and Application of the Reconstruction Software for the BaBar Calorimeter
}

\author{
Philip David Strother \\ Stanford Linear Accelerator Center \\ Stanford University \\ Stanford, CA 94309
}

SLAC-Report-828

Prepared for the Department of Energy under contract number DE-AC02-76SF00515

Printed in the United States of America. Available from the National Technical Information Service, U.S. Department of Commerce, 5285 Port Royal Road, Springfield, VA 22161. 
This document, and the material and data contained therein, was developed under sponsorship of the United States Government. Neither the United States nor the Department of Energy, nor the Leland Stanford Junior University, nor their employees, nor their respective contractors, subcontractors, or their employees, makes an warranty, express or implied, or assumes any liability of responsibility for accuracy, completeness or usefulness of any information, apparatus, product or process disclosed, or represents that its use will not infringe privately owned rights. Mention of any product, its manufacturer, or suppliers shall not, nor is it intended to, imply approval, disapproval, or fitness of any particular use. A royalty-free, nonexclusive right to use and disseminate same of whatsoever, is expressly reserved to the United States and the University. 


\title{
Design and application of the reconstruction software for the BaBar calorimeter
}

\author{
Philip David Strother
}

Imperial College, November 1998

A thesis submitted for the degree of Doctor of Philosophy

of the University of London, and Diploma of Imperial College 


\begin{abstract}
The BaBar high energy physics experiment will be in operation at the PEP-II asymmetric $e^{+} e^{-}$ collider in Spring 1999. The primary purpose of the experiment is the investigation of CP violation in the neutral $B$ meson system. The electromagnetic calorimeter forms a central part of the experiment and new techniques are employed in data acquisition and reconstruction software to maximise the capability of this device.

The use of a matched digital filter in the feature extraction in the front end electronics is presented. The performance of the filter in the presence of the expected high levels of soft photon background from the machine is evaluated.

The high luminosity of the PEP-II machine and the demands on the precision of the calorimeter require reliable software that allows for increased physics capability. $\mathrm{BaBar}$ has selected $\mathrm{C}++$ as its primary programming language and object oriented analysis and design as its coding paradigm. The application of this technology to the reconstruction software for the calorimeter is presented. The design of the systems for clustering, cluster division, track matching, particle identification and global calibration is discussed with emphasis on the provisions in the design for increased physics capability as levels of understanding of the detector increase.

The CP violating channel $B^{o} \rightarrow J / \Psi K_{s}^{o}$ has been studied in the two lepton, two $\pi^{0}$ final state. The contribution of this channel to the evaluation of the angle $\sin 2 \beta$ of the unitarity triangle is compared to that from the charged pion final state. An error of 0.34 on this quantity is expected after 1 year of running at design luminosity.
\end{abstract}




\section{Contents}

1 CP violation in the $B$ meson system $\quad 15$

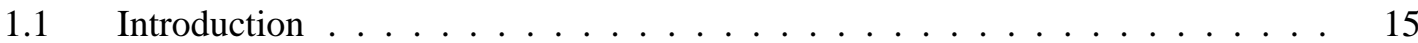

1.2 General phenomenology of mixing of neutral mesons . . . . . . . . . . 15

$1.3 \quad C P$ violation and mixing in the $K$ meson system $\ldots \ldots \ldots \ldots \ldots$

1.3.1 Direct $C P$ violation . . . . . . . . . . . . . . . . 20

$1.4 C P$ violation and mixing in the $B$ meson system $\ldots \ldots \ldots \ldots \ldots \ldots$

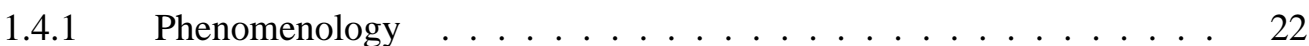

1.4.2 $C P$ violation in $B$ decays $\ldots \ldots \ldots \ldots . \ldots \ldots 22$

1.4.2.1 Direct $C P$ violation . . . . . . . . . . . 22

1.4.2.2 CP violation in mixing $\ldots \ldots \ldots 23$

1.4.2.3 CP violation in the interference between decays . . . . 24

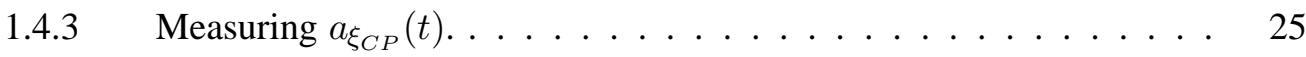

1.5 Quark mixing and the CKM matrix .............. 26

1.5.1 The CKM description of $C P$ violation . . . . . . . . . . 26

1.6 CKM Status and Current Constraints . . . . . . . . . . . . . . . . . . 32

$1.7 \quad$ Chapter Summary . . . . . . . . . . . . . . . . . . . . . 33

2 The BaBar experiment and the PEP-II storage ring 35

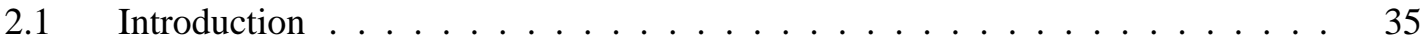

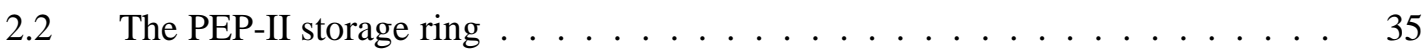

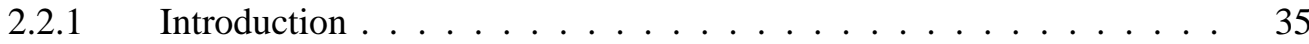

2.2 .2 The Main Storage Ring . . . . . . . . . . . . . . . . . . 36

2.2 .3 The Injection System . . . . . . . . . . . . . . . . . . . 38 
2.2.4 The Interaction Region . . . . . . . . . . . . . . . . . . . . 39

2.2.5 Machine backgrounds . . . . . . . . . . . . . . 41

2.2.5.1 Synchrotron radiation background . . . . . . . . 41

2.2.5.2 Lost beam particle backgrounds . . . . . . . . . . 41

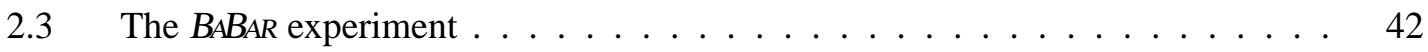

2.3.1 The Silicon Vertex Tracker . . . . . . . . . . . . . . . 44

2.3.1.1 Overview .................. 44

2.3.1.2 Detector Layout . . . . . . . . . . . . . . . 44

2.3.1.3 Electronics and readout . . . . . . . . . . 46

2.3.2 The Drift Chamber . . . . . . . . . . . . . . . . . . . 46

2.3.2.1 Mechanical design $\ldots \ldots \ldots \ldots$. . . . . . . 46

2.3.2.2 Electronics . . . . . . . . . . . . . . . . 48

2.3 .3 The DIRC . . . . . . . . . . . . . . . . . . . . . . . 49

2.3.3.1 Mechanical construction . . . . . . . . . . . 49

2.3.3.2 Readout .................. 50

2.3.4 The Electromagnetic Calorimeter . . . . . . . . . . . . . . 51

2.3.5 The Instrumented Flux Return . . . . . . . . . . . . . . . 52

2.3.5.1 RPC construction and readout $\ldots \ldots \ldots 54$

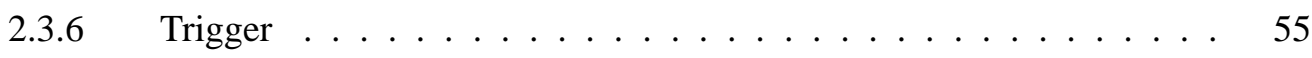

2.4 Chapter Summary . . . . . . . . . . . . . . . . . . . . . 56

3 Software for the Electromagnetic Calorimeter $\quad 57$

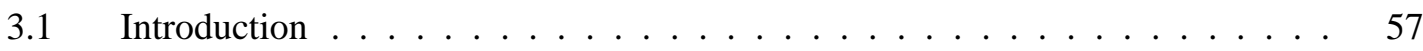

$3.2 \mathrm{C}++$ and object oriented design $\ldots \ldots \ldots \ldots \ldots$

3.2.1 The Concept of an Object . . . . . . . . . . . . 57

$3.2 .2 \quad$ Abstraction $\ldots \ldots \ldots \ldots \ldots \ldots$

3.2.3 Encapsulation . . . . . . . . . . . . . . . . 59

3.2.4 Object Oriented Design . . . . . . . . . . . . . . . . . . 59 
3.2.5 Relevance to physics capability . . . . . . . . . . . 59

3.2.6 The BABAR Framework Software . . . . . . . . . . . . . 60

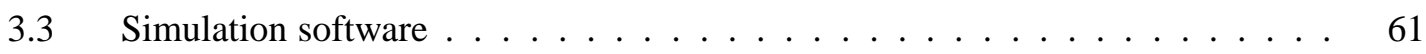

3.3.1 The calorimeter electronics simulation . . . . . . . . . 61

3.3.2 Digital filtering . . . . . . . . . . . . . . 64

3.3.2.1 Theory of the Matched Filter . . . . . . . . 64

3.3.2.2 Digital filter performance . . . . . . . . . 67

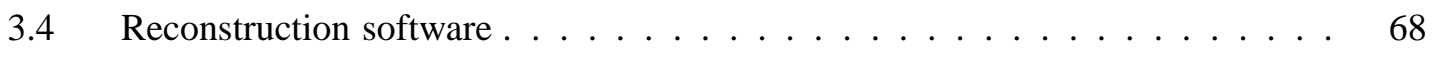

3.4.1 Basic reconstruction $\ldots \ldots \ldots \ldots 6$

3.4.1.1 Clustering .................. 68

3.4.1.2 Bump splitting . . . . . . . . . . . . . 69

3.4.2 Track-cluster matching . . . . . . . . . . . . 70

3.4.3 Particle identification . . . . . . . . . . . . 71

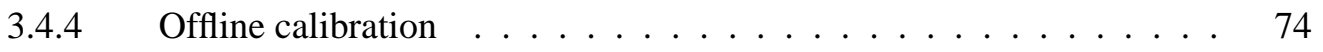

3.4.4.1 Retrieval ................ 75

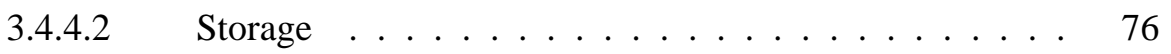

3.4.4.3 Offline calibration: summary . . . . . . . . 77

3.5 Chapter summary . . . . . . . . . . . . . . . . 77

4 Neutral particles in the electromagnetic calorimeter 79

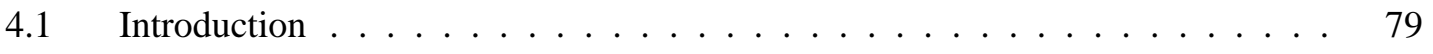

$4.2 \quad$ Reconstruction parameters . . . . . . . . . . . . . . 81

4.2.1 Clustering parameters $\ldots \ldots \ldots \ldots 1$

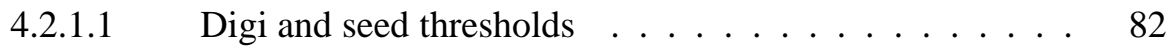

4.2.1.2 Seed and cluster thresholds . . . . . . . . . . 84

4.2.2 The CLEO clustering algorithm . . . . . . . . . . . . . . 84

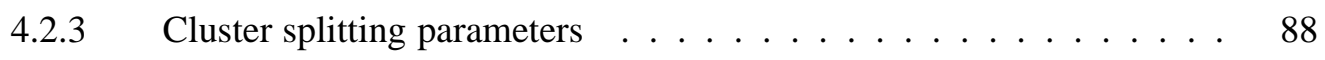

$4.3 \quad \pi^{0}$ and photon identification $\ldots \ldots \ldots \ldots \ldots$ 
4.3.1 Discriminating variables .................. 90

4.4 Chapter Summary . . . . . . . . . . . . . . . . . . . . . . . . 95

5 Study of the channel $B^{0} \rightarrow J / \psi K_{S}^{0} \rightarrow l^{+} l^{-} \pi^{0} \pi^{0}$

$5.1 \quad$ Introduction . . . . . . . . . . . . . . . . . . 97

5.2 Reconstructing the $J / \psi \ldots \ldots \ldots \ldots \ldots \ldots$

$5.2 .1 \quad$ Tracking requirements $\ldots \ldots \ldots . \ldots . \ldots 98$

$5.2 .2 \quad J / \psi$ reconstruction $\ldots \ldots \ldots \ldots 9 . \ldots \ldots$

5.3 Reconstructing the $K_{S}^{0} \ldots \ldots \ldots \ldots \ldots \ldots \ldots$

5.4 Reconstructing the $\mathrm{B}^{0} \ldots \ldots \ldots \ldots \ldots \ldots$. . . . . . . . . . . 104

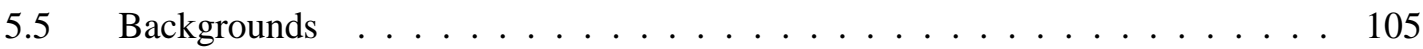

5.5.1 Combinatorial backgrounds and $J / \psi K_{L}^{0} \ldots \ldots \ldots \ldots$

5.5.2 Physics backgrounds . . . . . . . . . . . . . . 106

5.5.3 Machine backgrounds . . . . . . . . . . . . . 107

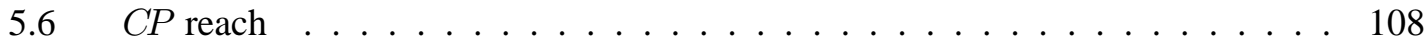

$5.6 .1 \quad$ Measuring $\Delta t \ldots \ldots \ldots \ldots \ldots \ldots \ldots$

5.6.2 Determination of the $b$ quark flavour . . . . . . . . . . . . . 110

$5.6 .3 \quad C P$ reach $\ldots \ldots \ldots \ldots \ldots \ldots \ldots \ldots$

5.7 Chapter Summary . . . . . . . . . . . . . . . . . . . . . . 111

6 Conclusions $\quad 113$

$\begin{array}{lll}\text { A The Wigner-Weisskopf formalism } & 121\end{array}$

$\begin{array}{ll}\text { B Class diagrams } & 125\end{array}$ 


\section{List of Figures}

1-1 $B$ meson decay rates as a function of lifetime for a $C P$ asymmetry of $\operatorname{Im}(\lambda)=$ 0.75. If no $C P$ violation were observed, the two decay rates would follow the exponential (solid line) . . . . . . . . . . . . . . . . . .

1-2 Unitarity triangles in the complex plane. Figure (a) depicts the triangle relating to possible CP violation in B physics. Figure (b) is the same triangle in the Wolfenstein parameterisation. . . . . . . . . . . . . .

1-3 Figure (a) depicts a typical process for $\mathrm{B}^{0}$ mixing. Figure (b) is a typical decay,

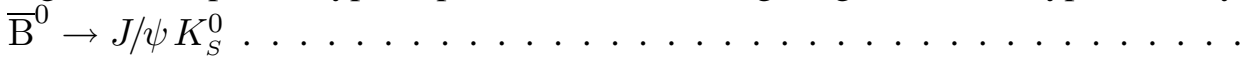

1-4 Allowed regions in the $\rho, \eta$ plane from current measurements. The dotted lines represent the boundaries of the allowed regions by allowing all parameters to vary. The contours, centred with a dot, represent the $95 \%$ confidence limits region using a given set of theoretical parameters. Limits from $\Delta m_{B_{s}}$ are not included. . . . .

2-1 Synchrotron radiation deposits from the low energy beam (top) and high energy beam (bottom). The Q1 quadrupole, being off axis w.r.t the low energy beam, and the B1 separation dipoles are the principle sources of synchrotron radiation at the IP. The darker shading indicates regions of higher photon density. . . . . . . . .

2-2 The BABAR detector. The $9.0 \mathrm{GeV}$ electron beam travels left to right, the $3.1 \mathrm{GeV}$ positron beam right to left. The interaction point is marked by the crosshairs. . .

2-3 The BABAR silicon vertex tracker. Cross sectional view in the $r / \phi$ plane. The beam pipe is shown in the centre. . . . . . . . . . . . . .

2-4 The BABAR silicon vertex tracker. Cross sectional view in the $z$ plane. The interaction point is marked, indicating the asymmetry of PEP-II. The structures to the right and left are the beam separating fixed dipole magnets. . . . . . . . . . .

2-5 The BABAR drift chamber ( $z$ projection). The chamber is offset in $z$ by $367 \mathrm{~mm}$ to account for the asymmetry. The dimensions shown are in $\mathrm{mm} . \ldots$. . . . . . .

2-6 Section of the cell layout for the drift chamber. Axial layers are labeled A, stereo layers $\mathrm{U}$ and $\mathrm{V} . \ldots \ldots \ldots \ldots \ldots$

2-7 The BABAR DIRC concept. Čerenkov radiation is internally reflected in the quartz bar, and emitted at the end of the bar preserving the Čerenkov angle. . . . . . . . 
2-8 Cross sectional view of the electromagnetic calorimeter showing angular coverage. The electron beam has the higher energy of the two. . . . . . . . . . .

2-9 End view of the barrel section of the instrumented flux return, showing integration with the solenoid. The inner resistive plate chamber (iRPC) is not shown. . . . .

2-10 Cross section of the layout of a typical RPC used in BABAR's instrumented flux return. 54

3-1 CR-RC-RC $\left(C R-R C^{2}\right)$ shaping circuit for the photodiode readout. The differentiation $(C R)$ stage has a time constant of $0.8 \mu \mathrm{s}$ and the integration $(R C)$ stages have identical time constants of $0.25 \mu \mathrm{s}$. The input to this circuit is the amplified photodiode output. The output from this circuit is fed to the ADC circuit. . . . .

3-2 Energy deposit in the calorimeter at times ten nominal machine background, summed over all crystals. . . . . . . . . . . . . . . . . . . . . . . 64

3-3 Individual crystal noise as function of machine background. The upper points represent a classical analogue readout with a shaping time of $2 \mu \mathrm{s}$ such as that used in CLEO. The lower points represent a digitally filtered readout using a short shaping time. It can be seen that the noise from soft photon machine background dominates over electronics noise (known to be about $150 \mathrm{keV}) . \ldots$. . . . . .

3-4 Resolution for $320 \mathrm{MeV}$ photons at times ten nominal background with classical feature extraction (left) and matched digital filter feature extraction (right). . . . .

4-1 Fraction of $\pi^{0} \mathrm{~s}$ whose daughter photons are sufficiently close to form only one cluster as a function of energy. . . . . . . . . . . . . . . . . .

4-2 Photon energy spectrum for generic $B$ decays at $B A B A R$ (upper plot) and from the most energetic decay, $B \rightarrow \pi^{0} \pi^{0}$ (lower plot). The sturcture in the lower plot is purely statistical. . . . . . . . . . . . . . .

4-3 Efficiency for $\mathrm{B}^{0}$ reconstruction (solid line) and $\pi^{0}$ reconstruction (dashed line) versus minimum detectable photon energy. . . . . . . . . . . . . . . .

4-4 Number of observed clusters above $20 \mathrm{MeV}$ for ten times nominal machine background, for sparsification cuts of $5 \mathrm{MeV}$ (top) and $10 \mathrm{MeV}$ (bottom), and various seed crystal and digi energy cuts. The points have been separated horizontally for clarity. . . . . . . . . . . . . . . . . . .

4-5 Energy spectrum of clusters from ten times nominal background, using standard

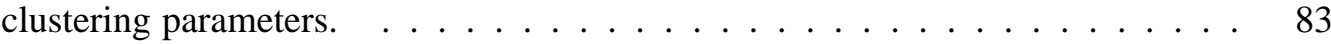

4-6 Number of observed clusters at times ten nominal background. . . . . . . . . . . 84 
4-7 Comparison of the CLEO clustering algorithm's resolution to the projected performance for the $B A B A R$ algorithm. Each set of four points corresponds to (left to right): CLEO algorithm taking all crystals (denoted $\triangle$ ), CLEO algorithm with the number of crystals limited according to the algorithm described in the text $(\square)$, BaBar algorithm taking all crystals $(\diamond)$, and BaBar algorithm with a limited number of crystals $(O)$. The top plot assumes no machine background, the middle nominal levels and the lower plot ten times nominal levels. $40 \mathrm{MeV}$ photons have too poor a resolution at ten times nominal background to be described well by the function given in the text and are omitted from the above plot. . . . . . . . .

4-8 Comparison of the CLEO clustering algorithm's efficiency to the projected performance for the $B A B A R$ algorithm. Each set of four points corresponds to (left to right): CLEO algorithm taking all crystals $(\triangle)$, CLEO algorithm with a limited number of crystals $(\square)$, BaBar algorithm taking all crystals $(\diamond)$, and BaBar algorithm with a limited number of crystals $(O)$. The top plot assumes no machine background, the middle nominal levels and the lower plot ten times nominal levels. At nominal background, the $40 \mathrm{MeV}$ photon resolution is such that too many machine background photons would be included in the efficiency calculation as described. The resolution (and therefore efficiency) cannot be determined at ten times nominal background for $40 \mathrm{MeV}$ photons. . . . . . . . . . . . .

4-9 Comparison of the CLEO and BABAR clustering algorithms' mass resolution for $500 \mathrm{MeV} \pi^{0}$ s. . . . . . . . . . . . . . . . . . .

4-10 The comparison of the highest energy crystal in a shower to the highest of its neighbours for medium energy $(320 \mathrm{MeV})$ photons. (Left-bottom) The ratio of the highest energy crystal to the highest of its neighbours. (Top) The inverse of this quantity. (Right) The number of photon clusters whose highest energy crystal/highest energy neighbour crystal ratio exceeds a given value. . . . . . . .

4-11 The comparison of the highest energy crystal in a shower to the average of its neighbours for medium energy $(320 \mathrm{MeV})$ photons. (Left-bottom) The ratio of the highest energy crystal to the average of its neighbours. (Top) The inverse of this quantity. (Right) The number of photon clusters whose highest energy crystal/average energy of neighbours ratio exceeds a given value. . . . . . . .

4-12 Number of maxima observed in one cluster for $320 \mathrm{MeV}$ photons as a function of the ratio between the candidate maximum and (left) the highest energy neighbouring crystal and (right) the average energy of neighbouring crystals. . . . . . . .

4-13 Lateral profile of photon clusters. The horizontal axis shows the lateral distance from the centroid in $\mathrm{cm}$ at the nominal shower maximum, and the vertical axis the fraction of the cluster energy contained outside that distance. Clockwise from top left are the profiles for $160 \mathrm{MeV}, 320 \mathrm{MeV}, 2.56 \mathrm{GeV}$ and $1.28 \mathrm{GeV}$ photons. . . . 
4-14 Distribution of the second moment of clusters in a $3 \mathrm{GeV} \pi^{0}$ sample. A clear separation of those $\pi^{0} \mathrm{~s}$ forming one cluster from those creating two (single photon) clusters is demonstrated by using the Monte-Carlo truth information. . . . . . . .

4-15 Average squared deviation (in $\mathrm{GeV}^{2}$ ) of the observed energy of crystals in a cluster from the electromagnetic prediction for $1.28 \mathrm{GeV}$ photons (top) and $4 \mathrm{GeV}$ $\pi^{0} \mathrm{~s}$ (bottom). Different, but not well separated, distributions are observed. . . . .

4-16 Second moment distributions for $2 \mathrm{GeV} \pi^{0} \mathrm{~s}$. The fit function is described in the text. The peak position varies as $\sim 1 / E^{2}$.

4-17 Efficiency of identification against probability of mis-identification for photons at various confidence levels for the observed energy deposit to be compatible with the photon hypothesis. . . . . . . . . . . . . . . .

4-18 Efficiency of identification against probability of mis-identification for $\pi^{0} \mathrm{~s}$ at various confidence levels for the observed energy deposit to be compatible with the

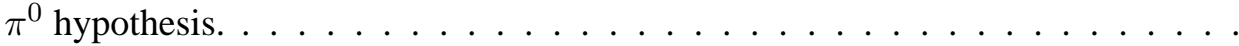

5-1 Tree (a) and penguin (b) diagrams for $\mathrm{B}^{0} \rightarrow J / \psi K_{S}^{0} \ldots \ldots \ldots \ldots$

5-2 $J / \psi$ efficiency as a function of upper and lower mass cut. The effects of acceptance are excluded. In each case the other mass cut is held constant at the nominal

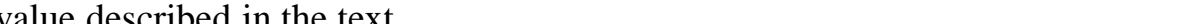

5-3 $J / \psi$ mass resolution. The fit is to the Novosibirsk function (see chapter 4) to account for the lower mass caused by electron bremsstrahlung. The width is

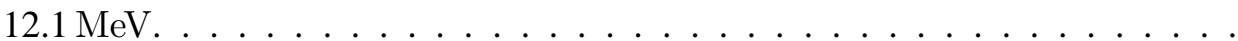

5-4 Mass and centre of mass momentum distribution for signal $J / \psi$ candidates from 2000 simulated events. . . . . . . . . . . . . . . . . . . . 101

5-5 Fake $J / \psi$ mass and momentum distributions from (left) continuum and (right) generic $B$ meson decays. 10000 events were simulated. . . . . . . . . . . . .

5-6 Two cluster invariant mass distribution for all cluster pairs (left) and for signal

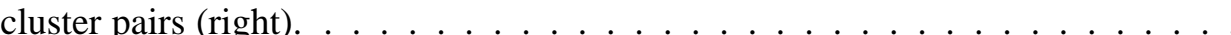

5-7 (Left) Distribution of the $K_{S}^{0}$ decay points as determined by the formation of the best $\pi^{0}$ mass for a cluster pair. The top plot is for $\pi^{0} \mathrm{~s}$ coming from signal $K_{S}^{0} \mathrm{~s}$, the lower from fake $K_{S}^{0}$ candidates. The spikes at -2 and +5 in the lower plot are caused by those candidates for which a best mass could not be determined between these times. (Right) Distribution of the absolute difference between the two decay points determined for each candidate. Again the top plot is for signal $K_{S}^{0}$, and the lower one for fake $K_{S}^{0}$ candidates. The small spike at 7.0 is due to those candidates whose best decay points were at the extremes of the range described above. . . . 
5-8 Distribution of centre of mass momentum of (left) all $K_{S}^{0}$ candidates and (right) signal candidates. . . . . . . . . . . . . . . . . . . . . 104

5-9 $K_{S}^{0}$ mass distribution calculated at the decay vertex for non-signal (left) and signal (right) combinations. . . . . . . . . . . . . . . . . . . . 105

5-10 Origin of the photons in fake $B$ candidates in 2000 signal events. The number of photons that originated from the signal $K_{S}^{0}$ and subsequently used to construct the $B$ candidate is shown. Those candidates with 4 such photons have the correct $K_{S}^{0}$ but a fake $J / \psi$. Candidates with 0 such photons have the $K_{S}^{0}$ constructed entirely from photons from the other $B$ meson decay in the event. It can be seen that the large majority of events require at least one photon from the signal $K_{S}^{0}$ in order to fake a $B$ candidate. . . . . . . . . . . . . . . . . . . . . . 106

5-11 The $z$ resolution of the $J / \psi$ vertex. The fit is to two Gaussians, the upper having a width of $45 \mu \mathrm{m}$, the lower $320 \mu \mathrm{m}$. The ratio of areas is $3.5: 1 \ldots \ldots \ldots$. . . .

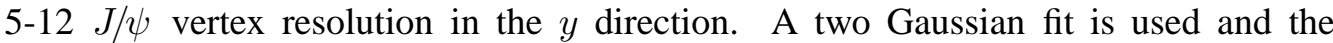
resulting widths are 45 and $221 \mu \mathrm{m}$ with area ratios 20:3. This is considerably larger than the vertical beam size. . . . . . . . . . . . . . .

5-13 Tag side vertex resolution. A two Gaussian fit yields widths of $108 \mu \mathrm{m}$ and $316 \mu \mathrm{m}$, with an area ratio 1.1:1.0. . . . . . . . . . . . 110

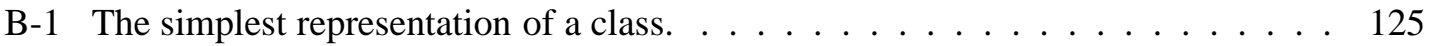

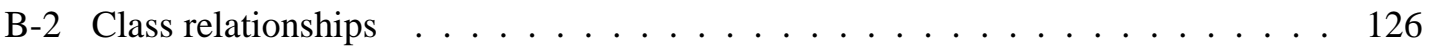

B-3 Class diagram for the particle ID framework for the calorimeter. It is discussed more fully in chapter $3 \ldots \ldots \ldots \ldots \ldots$

B-4 Class diagram for the offline, global calibration system. This diagram refers to the retrieval side of the system. It is discussed more fully in chapter 3 . . . . . . . . 128

B-5 Class diagram for the offline, global calibration system. This diagram refers to the storage side of the system. It is discussed more fully in chapter $3 \ldots$. . . . . . . 


\section{List of Tables}

2-1 Parameters of the PEP-II asymmetric storage ring. . . . . . . . . . . 36

2-2 Occupancy rates and radiation doses from lost beam particle backgrounds in the four innermost sections of the BABAR experiment. . . . . . . . . . . . . 42

2-3 Description of the layers of the silicon vertex tracker. . . . . . . . . . 44

2-4 Description of the wafers constituting the silicon vertex tracker. . . . . . . . . 45

2-5 Description of the drift chamber superlayers. . . . . . . . . . . . 47

2-6 Properties of crystal CsI(Tl) scintillator. . . . . . . . . . . . . . . . 51

2-7 Trigger efficiency for various physics channels to be studied with the $B A B A R$ detector. 55

5-1 Relative reconstruction efficiency for the channel $B \rightarrow J / \psi K_{S}^{0} \rightarrow l^{+} l^{-} \pi^{0} \pi^{0}$ as a function of cluster energy cut at nominal machine background . . . . . . . . 107 


\section{Acknowledgments}

Firstly I would like to thank Professor Peter Dornan for inviting me to work in Imperial's High Energy Physics group and my supervisor Jordan Nash for all his help and assistance, and for proving that it is possible for an American to understand the laws of cricket.

The $B A B A R$ experiment has been an exciting environment in which to work and I have had the pleasure of collaboration with a wide variety of people. I have been particularly fortunate in the colleagues with whom I have been working most closely. Bob Jacobsen, Ed Frank, Steve Gowdy, Helmut Marsiske, Paul Harrison and Pete Sanders have all unfailingly provided great insight, gentle correction and perhaps without them knowing, not a little inspiration, all delivered with a sense of humour. It has been a pleasure and an honour to work with them.

Pete, Paul Dauncey, Alex Howard and Roly Martin get a huge thank you for reading, correcting and offering advice on this thesis. It's been an "interesting" time. All remaining errors are therefore the fault of London Underground, who apologise for any inconvenience.

High energy physics has been described ("condemned"??) as an international beer drinking conspiracy. I am indebted to those people (Dave, Pete, Roly, Steve, Colin) who introduced me to this concept and for their sterling efforts in perpetuation of this rumour. May the pumps never run dry.

To all my friends at IC and SLAC that are too many to mention individually, thanks for some great moments. Particular mention though must be made of the NATO ASI crew ( $151^{\circ}$ proof rum: "Don't do that!") and to my year colleagues here at IC—Jan, Jo and Suzy—for many a beer and coffee break, and for achieving the near impossible in making the terminal room a brighter place to work.

Five years ago in Paris I caught the HEP bug. For this I blame and thank wholeheartedly Patrick Roudeau of LAL. First teachers do not come much better.

Finally there are those without whose support the last three years would not have been possible. To those at St. Helen's church, in particular Chris, Neil \& Rachel and Nick \& Rachael, for all the support, good food and patient advice-thanks: 2 Th 3:16. To my flatmates: Al, Rob and Lucy - thanks for the good company, for putting up with the eccentric hours and proving that it is possible for 3 final year $\mathrm{PhD}$ students and someone holding down two jobs to live together without bloodshed. To my Mum, sister Karen, Dad and recently added family: Sue, Andrew, Martin and Zöe- thank you for all your unending support and encouragement: there are not really the words to express my gratitude. Finally to Giulia, for showing me that not only is there a life outside HEP but it is a beautiful one inhabited by the likes of Neruda, Chagall, Galeano, Haring, and not forgetting the occasional monster. Thank you for sharing it with me. 


\section{CP violation in the $B$ meson system}

\subsection{Introduction}

$C P$ violation is one of the least well measured aspects of the standard model. To date it has only been observed in one system, that of the neutral $K$ mesons[1]. It is, however, one of the necessary conditions needed to explain the baryon asymmetry of the universe[2] and is an important aspect of the CKM picture of quark mixing[3] which first demonstrated the necessity of at least three quark generations in order that the observed $C P$ violation be accommodated.

In this chapter a description of generic neutral meson mixing will be given, which will then be applied to the $K$ meson system in order to illustrate how mixing can give rise to $C P$ violation, and also to show the other possible sources of this phenomenon. These will be explained in greater depth in section 1.4 , where the $B$ meson system is considered. Finally an introduction to the concept of quark mixing and the CKM picture of $C P$ violation will be given.

\subsection{General phenomenology of mixing of neutral mesons}

One possible cause of the phenomenon of CP violation in the $K$ meson and $B$ meson system is the mixing of particle-antiparticle states that occurs in each system. The formalism is the same in both cases and so it is described here for a generic neutral meson $P^{0}$, which is capable of mixing with its antiparticle $\overline{P^{0}}$. A full discussion may be found in [4].

For a generic state, $\Psi$, consisting of a combination of the two $P$ states, one may write

$$
\Psi(t)=\left|P^{0}(\mathbf{p}, t)\right\rangle \otimes\left|\overline{P^{0}}(-\mathbf{p}, t)\right\rangle+C\left|P^{0}(-\mathbf{p}, t)\right\rangle \otimes\left|\overline{P^{0}}(\mathbf{p}, t)\right\rangle .
$$

where $C$ denotes the charge conjugation phase. The time evolution of the individual states $P^{\infty}$ and and $\overline{P^{0}}$ is governed by the Wigner-Weisskopf result from elementary quantum mechanics, derived in appendix A. This states that for a generic state $|\Phi(t)\rangle$ decaying into stable states $|\beta\rangle$ from unstable states $|\alpha\rangle$

$$
|\Phi(t)\rangle=\sum_{\alpha} \phi_{\alpha}(t)|\alpha\rangle+\sum_{\beta} c_{\beta}(t)|\beta\rangle,
$$

the time dependence of $\phi_{\alpha}(t)$ is given by

$$
\phi_{\alpha}(t)=e^{(-i \widehat{\mathcal{M}} t)} \phi_{\alpha}(0)
$$


The mass matrix $\widehat{\mathcal{M}}$ is a $2 \times 2$ matrix which has its origins in the Hamiltonian that governs the transition between the two states. In general it is not Hermitian, but may be broken down into two Hermitian parts:

$$
\begin{aligned}
M & =\frac{1}{2}\left(\widehat{\mathcal{M}}+\widehat{\mathcal{M}}^{\dagger}\right) \\
\Gamma & =i\left(\widehat{\mathcal{M}}-\widehat{\mathcal{M}}^{\dagger}\right) .
\end{aligned}
$$

In general the flavour eigenstates $P^{0}$ and $\overline{P^{0}}$ will not be eigenvectors of the mass matrix. Instead, the mass eigenstates will be linear combinations of these. In the case where the charge conjugation phase $C=-1$, the mass eigenstates may be written:

$$
\begin{aligned}
& \left|P_{1}^{0}\right\rangle=p\left|P^{0}\right\rangle+q\left|\overline{P^{0}}\right\rangle \\
& \left|P_{2}^{0}\right\rangle=p\left|P^{0}\right\rangle-q\left|\overline{P^{0}}\right\rangle
\end{aligned}
$$

where

$$
\begin{aligned}
\widehat{\mathcal{M}}\left|P_{1}^{0}\right\rangle & =\left(m_{1}-i \frac{\Gamma_{1}}{2}\right)\left|P_{1}^{0}\right\rangle=M_{1}\left|P_{1}^{0}\right\rangle \\
\widehat{\mathcal{M}}\left|P_{2}^{0}\right\rangle & =\left(m_{2}-i \frac{\Gamma_{2}}{2}\right)\left|P_{2}^{0}\right\rangle=M_{2}\left|P_{2}^{0}\right\rangle .
\end{aligned}
$$

and $p, q$ are complex numbers normalised such that $|p|^{2}+|q|^{2}=1$. The time dependence of the flavour eigenstate $P^{0}$ is then given by

$$
\begin{aligned}
\left|P^{0}(t)\right\rangle & =\frac{1}{2 p}\left(e^{-i M_{1} t}\left|P_{1}^{0}\right\rangle+e^{-i M_{2} t}\left|P_{2}^{0}\right\rangle\right) \\
& =\frac{1}{2 p}\left[e^{-i M_{1} t}\left(p\left|P^{0}\right\rangle+q\left|\overline{P^{0}}\right\rangle\right)+e^{-i M_{2} t}\left(p\left|P^{0}\right\rangle-q\left|\overline{P^{0}}\right\rangle\right)\right] \\
& =f_{+}(t)\left|P^{0}\right\rangle+\frac{q}{p} f_{-}(t)\left|\overline{P^{0}}\right\rangle
\end{aligned}
$$

where

$$
f_{ \pm}(t)=\frac{1}{2} \exp \left(\left\{-i m+\frac{\Gamma}{2}\right\} t\right)\left[\exp \left(+\left\{i \Delta m-\frac{\Delta \Gamma}{2}\right\} \frac{t}{2}\right) \pm \exp \left(-\left\{i \Delta m-\frac{\Delta \Gamma}{2}\right\} \frac{t}{2}\right)\right]
$$

where $\Delta m=m_{1}-m_{2}$ and $\Delta \Gamma=\Gamma_{1}-\Gamma_{2}$. Using CPT invariance, which gives that $\widehat{\mathcal{M}}_{11}=\widehat{\mathcal{M}}_{22}$, and the Hermitian property of the matrices $M$ and $\Gamma$, the eigenvalue equations for $p$ and $q$ give the results:

$$
\begin{aligned}
(\Delta m)^{2}-\frac{1}{4}(\Delta \Gamma)^{2} & =4\left(\left|M_{12}\right|^{2}-\frac{\left|\Gamma_{12}\right|^{2}}{4}\right), \\
(\Delta m)(\Delta \Gamma) & =-4 \operatorname{Re}\left(M_{12} \Gamma_{12}^{*}\right), \\
\frac{q}{p} & =-\frac{\Delta m-i \frac{\Delta \Gamma}{2}}{2\left(M_{12}-i \frac{\Gamma_{12}}{2}\right)} .
\end{aligned}
$$

The specialisations of these formulae to the $K$ meson system are discussed in the next section, while those relating to the $B$ meson system are discussed in section 1.4. 


\section{3 $C P$ violation and mixing in the $K$ meson system}

The discovery of CP violation in the kaon system by Christiansen et al. in 1964 [1] was the first evidence for CP not being an exact symmetry. This section gives an overview of the phenomenology of the system, using the formalism described above. For more complete reviews, see for example Wolfenstein[5, 6], Mannelli [7] or Kleinknecht [8].

$\mathrm{K}^{0}$ and $\overline{\mathrm{K}}^{0}$ are strangeness eigenstates with eigenvalues +1 and -1 respectively. In section $1.2 \mathrm{a}$ relative charge conjugation phase of $C=-1$ was assumed. Here a more general phase is assumed, and eigenstates are given by $\left|K_{L}^{0}\right\rangle$ and $\left|K_{S}^{0}\right\rangle$ such that

$$
\begin{aligned}
\left|K_{L}^{0}\right\rangle & =\frac{r\left|\mathrm{~K}^{0}\right\rangle+s\left|\overline{\mathrm{K}}^{0}\right\rangle}{\sqrt{|r|^{2}+|s|^{2}}}, \\
\left|K_{S}^{0}\right\rangle & =\frac{p\left|\mathrm{~K}^{0}\right\rangle+q\left|\overline{\mathrm{K}}^{0}\right\rangle}{\sqrt{|p|^{2}+|q|^{2}}}
\end{aligned}
$$

where $p, q, r$ and $s$ are complex numbers. The two states are referred to as short $(S)$ and long $(L)$, reflecting that the observed states have markedly different lifetimes [9]:

$$
\tau_{K_{S}^{0}}=8.927 \pm 0.009 \times 10^{-10} \mathrm{~s} ; \tau_{K_{L}^{0}}=5.17 \pm 0.04 \times 10^{-8} \mathrm{~s} .
$$

If $\widehat{\mathcal{M}}$ is represented by

$$
\widehat{\mathcal{M}}=\left(\begin{array}{ll}
m_{11}-\frac{i}{2} \Gamma_{11} & m_{12}-\frac{i}{2} \Gamma_{12} \\
m_{21}-\frac{i}{2} \Gamma_{21} & m_{22}-\frac{i}{2} \Gamma_{22}
\end{array}\right),
$$

then the eigenvalue equations for the mass matrix are

$$
\begin{aligned}
& r\left(m_{11}-\frac{i}{2} \Gamma_{11}\right)+s\left(m_{12}-\frac{i}{2} \Gamma_{12}\right)=r M_{L}, \\
& r\left(m_{21}-\frac{i}{2} \Gamma_{21}\right)+s\left(m_{22}-\frac{i}{2} \Gamma_{22}\right)=s M_{L}, \\
& p\left(m_{11}-\frac{i}{2} \Gamma_{11}\right)+q\left(m_{12}-\frac{i}{2} \Gamma_{12}\right)=p M_{S}, \\
& p\left(m_{21}-\frac{i}{2} \Gamma_{21}\right)+q\left(m_{22}-\frac{i}{2} \Gamma_{22}\right)=q M_{S},
\end{aligned}
$$

where

$$
\begin{aligned}
\widehat{\mathcal{M}}\left|K_{S}^{0}\right\rangle & =M_{S}\left|K_{S}^{0}\right\rangle, \\
\widehat{\mathcal{M}}\left|K_{L}^{0}\right\rangle & =M_{L}\left|K_{L}^{0}\right\rangle
\end{aligned}
$$

This gives the relation

$$
\left|\frac{r}{s}\right|^{2}=\frac{m_{12}-i \Gamma_{12} / 2}{m_{21}-i \Gamma_{21} / 2}=\left|\frac{p}{q}\right|^{2},
$$


i.e.

$$
\frac{p}{q}=\frac{r}{s} e^{-i \phi}
$$

where $\phi$ is an arbitrary phase constant, while

$$
\frac{r}{s}-\frac{p}{q}=\frac{\Delta M}{m_{21}-\frac{i}{2} \Gamma_{21}}
$$

where $\Delta M=M_{L}-M_{S}$. In order to determine the phase $\phi$, it is convenient to move to a basis defined by the $\mathrm{CP}$ eigenstates

$$
\begin{aligned}
& \left|K_{1}^{0}\right\rangle=\frac{1}{\sqrt{2}}\left(\left|\mathrm{~K}^{0}\right\rangle+\left|\overline{\mathrm{K}}^{0}\right\rangle\right), \\
& \left|K_{2}^{0}\right\rangle=\frac{1}{\sqrt{2}}\left(\left|\mathrm{~K}^{0}\right\rangle-\left|\overline{\mathrm{K}}^{0}\right\rangle\right) .
\end{aligned}
$$

In this basis

$$
\begin{aligned}
\left|K_{S}^{0}\right\rangle & =\frac{1}{\sqrt{1+\left|\varepsilon_{S}\right|^{2}}}\left(\left|K_{1}^{0}\right\rangle+\varepsilon_{S}\left|K_{2}^{0}\right\rangle\right), \\
\left|K_{L}^{0}\right\rangle & =\frac{e^{i \phi}}{\sqrt{1+\left|\varepsilon_{L}\right|^{2}}}\left(\left|K_{2}^{0}\right\rangle+\varepsilon_{L}\left|K_{1}^{0}\right\rangle\right) .
\end{aligned}
$$

CP violation will occur unless $\left\langle K_{S}^{0} \mid K_{L}^{0}\right\rangle=0$ and so to fix the phase one demands

$$
\left\langle K_{S}^{0} \mid K_{L}^{0}\right\rangle>0
$$

This gives the requirements

$$
m_{12}-\frac{i}{2} \Gamma_{12} \neq m_{21}-\frac{i}{2} \Gamma_{21}
$$

and

$$
e^{i \phi}\left(\varepsilon_{L}+\varepsilon_{S}^{*}\right)>0
$$

By invoking CPT invariance, it is possible to write

$$
\varepsilon_{S}=\varepsilon_{L}=\varepsilon,
$$

however in order to resolve the phase $\phi$, it is necessary to study the following semi-leptonic decays of the kaons:

$$
\begin{aligned}
& \Gamma\left(\mathrm{K}^{0} \rightarrow l^{-} \nu_{l} \pi^{+}\right) \\
& \Gamma\left(\mathrm{K}^{0} \rightarrow l^{+} \bar{\nu}_{l} \pi^{-}\right) \\
& \Gamma\left(\overline{\mathrm{K}}^{0} \rightarrow l^{-} \nu_{l} \pi^{+}\right) \\
& \Gamma\left(\overline{\mathrm{K}}^{0} \rightarrow l^{+} \bar{\nu}_{l} \pi^{-}\right)
\end{aligned}
$$


The $\Delta S=\Delta Q$ rule dictates that the decay widths 1.26 and 1.29 are zero, whilst CPT invariance implies that the widths 1.27 and 1.28 are equal. If one defines the quantities $A$ and $B$

$$
\begin{aligned}
A & =\Gamma\left(K_{L}^{0} \rightarrow l^{+} \overline{\nu_{l}} \pi^{-}\right), \\
& =\left\langle\mathrm{K}^{0} \mid K_{L}^{0}\right\rangle^{2} \Gamma\left(\mathrm{K}^{0} \rightarrow l^{+} \overline{\nu_{l}} \pi^{-}\right) \\
& =\frac{|1+\varepsilon|^{2}}{2\left(1+|\varepsilon|^{2}\right)} \Gamma\left(\mathrm{K}^{0} \rightarrow l^{+} \overline{\nu_{l}} \pi^{-}\right), \\
B & =\Gamma\left(K_{L}^{0} \rightarrow l^{-} \nu_{l} \pi^{+}\right) \\
& =\left\langle\overline{\mathrm{K}}^{0} \mid K_{L}^{0}\right\rangle^{2} \Gamma\left(\overline{\mathrm{K}}^{0} \rightarrow l^{-} \nu_{l} \pi^{+}\right) \\
& =\frac{|1-\varepsilon|^{2}}{2\left(1+|\varepsilon|^{2}\right)} \Gamma\left(\overline{\mathrm{K}}^{0} \rightarrow l^{-} \nu_{l} \pi^{+}\right),
\end{aligned}
$$

then

$$
\begin{aligned}
\delta & =\frac{A-B}{A+B} \\
& =\frac{2\left(\varepsilon^{*}+\varepsilon\right)}{2\left(1+|\varepsilon|^{2}\right)}, \\
& =\frac{2 \operatorname{Re}(\varepsilon)}{1+|\varepsilon|^{2}} .
\end{aligned}
$$

The quantity $\delta$ is measured to be greater than zero by experiment[9], so that the physical states may be represented by

$$
\begin{aligned}
\left|K_{S}^{0}\right\rangle & =\frac{1}{\sqrt{2\left(1+|\epsilon|^{2}\right)}}\left((1+\varepsilon)\left|\mathrm{K}^{0}\right\rangle+(1-\varepsilon)\left|\overline{\mathrm{K}}^{0}\right\rangle\right) \\
\left|K_{L}^{0}\right\rangle & =\frac{1}{\sqrt{2\left(1+|\epsilon|^{2}\right)}}\left((1+\varepsilon)\left|\mathrm{K}^{0}\right\rangle-(1-\varepsilon)\left|\overline{\mathrm{K}}^{0}\right\rangle\right) .
\end{aligned}
$$

From this one can write

$$
\begin{aligned}
\varepsilon & =\frac{p-q}{p+q} \\
& =\frac{(p / q)^{2}-1}{(p / q)^{2}+2 p / q+1} \\
& =\frac{\widehat{\mathcal{M}}_{12}-\widehat{\mathcal{M}}_{21}}{\widehat{\mathcal{M}}_{12}-\Delta M+\widehat{\mathcal{M}}_{21}} .
\end{aligned}
$$

If the $\mathrm{CP}$ violating terms are small, then one may approximate $\widehat{\mathcal{M}}_{12}+\widehat{\mathcal{M}}_{21} \simeq-\Delta M$, so finally $\varepsilon$ can be written as

$$
\varepsilon=\frac{m_{21}-m_{12}-i / 2\left(\Gamma_{21}-\Gamma_{12}\right)}{2\left(m_{L}-m_{S}\right)-i\left(\Gamma_{L}-\Gamma_{S}\right)} .
$$




\subsubsection{Direct $C P$ violation}

In addition to mixing, $\mathrm{CP}$ violation can occur because of differing decay amplitudes to the same final state. The $\mathrm{K}^{0} \rightarrow 2 \pi$ decay is an important example of this, given that the final state can have isospin values of $I=0$ or $I=2$. If $\widehat{\mathcal{T}}$ is the appropriate transition matrix, then the experimental observables are

$$
\begin{aligned}
\eta_{+-} & =\frac{\left\langle\pi^{+} \pi^{-}|\widehat{\mathcal{T}}| K_{L}^{0}\right\rangle}{\left\langle\pi^{+} \pi^{-}|\widehat{\mathcal{T}}| K_{S}^{0}\right\rangle} \\
\eta_{00} & =\frac{\left\langle\pi^{0} \pi^{0}|\widehat{\mathcal{T}}| K_{L}^{0}\right\rangle}{\left\langle\pi^{0} \pi^{0}|\widehat{\mathcal{T}}| K_{S}^{0}\right\rangle}
\end{aligned}
$$

These observables may be cast in terms of the variables

$$
\begin{aligned}
\varepsilon_{0} & =\frac{\left\langle\pi \pi, I=0|\widehat{\mathcal{T}}| K_{L}\right\rangle}{\left\langle\pi \pi, I=0|\widehat{\mathcal{T}}| K_{S}\right\rangle}, \\
\varepsilon_{2} & =\frac{1}{\sqrt{2}} \frac{\left\langle\pi \pi, I=2|\widehat{\mathcal{T}}| K_{L}\right\rangle}{\left\langle\pi \pi, I=0|\widehat{\mathcal{T}}| K_{S}\right\rangle}, \\
\omega & =\frac{\left\langle\pi \pi, I=2|\widehat{\mathcal{T}}| K_{S}\right\rangle}{\left\langle\pi \pi, I=0|\widehat{\mathcal{T}}| K_{S}\right\rangle} .
\end{aligned}
$$

Using the appropriate Clebsch-Gordan coefficients, one finds that the states representing $I=0$ and $I=2$ are given by

$$
\begin{aligned}
& \langle\pi \pi, I=0|=\frac{1}{\sqrt{3}}\left\langle\pi^{-} \pi^{+}\right|-\frac{1}{\sqrt{3}}\left\langle\pi^{0} \pi^{0}\right|+\frac{1}{\sqrt{3}}\left\langle\pi^{+} \pi^{-}\right| \\
& \langle\pi \pi, I=2|=\frac{1}{\sqrt{6}}\left\langle\pi^{-} \pi^{+}\right|-\sqrt{\frac{2}{3}}\left\langle\pi^{0} \pi^{0}\right|+\frac{1}{\sqrt{6}}\left\langle\pi^{+} \pi^{-}\right|,
\end{aligned}
$$

such that

$$
\begin{aligned}
\eta_{+-} & =\frac{\sqrt{2}\left\langle\pi \pi, I=0|\widehat{\mathcal{T}}| K_{L}\right\rangle+\left\langle\pi \pi, I=2|\widehat{\mathcal{T}}| K_{L}\right\rangle}{\sqrt{2}\left\langle\pi \pi, I=0|\widehat{\mathcal{T}}| K_{S}\right\rangle+\left\langle\pi \pi, I=2|\widehat{\mathcal{T}}| K_{S}\right\rangle} \\
& =\frac{\varepsilon_{0}+\varepsilon_{2}}{1+\omega / \sqrt{2}}, \\
\eta_{00} & =\frac{\left\langle\pi \pi, I=2|\widehat{\mathcal{T}}| K_{L}\right\rangle-\left\langle\pi \pi, I=0|\widehat{\mathcal{T}}| K_{L}\right\rangle / \sqrt{2}}{\left\langle\pi \pi, I=2|\widehat{\mathcal{T}}| K_{S}\right\rangle-\left\langle\pi \pi, I=0|\widehat{\mathcal{T}}| K_{S}\right\rangle / \sqrt{2}} \\
& =\frac{\varepsilon_{0}-2 \varepsilon_{2}}{1-\sqrt{2} \omega} .
\end{aligned}
$$

One may use the fact that the $I=2$ decay channels are heavily suppressed with respect to the $I=0$ channels. This gives $|\omega| \ll 1$ and

$$
\begin{aligned}
\eta_{+-} & =\varepsilon_{0}+\varepsilon_{2} \\
\eta_{00} & =\varepsilon_{0}-2 \varepsilon_{2} .
\end{aligned}
$$


One can then define a phase convention such that

so then

$$
\begin{aligned}
& \left\langle\pi \pi, I=0|\widehat{\mathcal{T}}| \mathrm{K}^{0}\right\rangle=A_{0} e^{i \delta_{0}}, \\
& \left\langle\pi \pi, I=0|\widehat{\mathcal{T}}| \overline{\mathrm{K}}^{0}\right\rangle=A_{0}^{*} e^{i \delta_{0}} \\
& \left\langle\pi \pi, I=2|\widehat{\mathcal{T}}| \mathrm{K}^{0}\right\rangle=A_{2} e^{i \delta_{2}}, \\
& \left\langle\pi \pi, I=2|\widehat{\mathcal{T}}| \overline{\mathrm{K}}^{0}\right\rangle=A_{2}^{*} e^{i \delta_{2}}
\end{aligned}
$$

$$
\begin{aligned}
\varepsilon_{0} & =\frac{\left\langle\pi \pi, I=0|\widehat{\mathcal{T}}| \mathrm{K}^{0}\right\rangle\left\langle\mathrm{K}^{0} \mid K_{L}^{0}\right\rangle+\left\langle\pi \pi, I=0|\widehat{\mathcal{T}}| \overline{\mathrm{K}}^{0}\right\rangle\left\langle\overline{\mathrm{K}}^{0} \mid K_{L}^{0}\right\rangle}{\left\langle\pi \pi, I=0|\widehat{\mathcal{T}}| \mathrm{K}^{0}\right\rangle\left\langle\mathrm{K}^{0} \mid K_{S}^{0}\right\rangle+\left\langle\pi \pi, I=0|\widehat{\mathcal{T}}| \overline{\mathrm{K}}^{0}\right\rangle\left\langle\overline{\mathrm{K}}^{0} \mid K_{S}^{0}\right\rangle} \\
& =\frac{\varepsilon\left(A_{0}+A_{0}^{*}\right)+A_{0}^{*}-A_{0}^{*}}{\varepsilon\left(A_{0}-A_{0}^{*}\right)+A_{0}+A_{0}^{*}} \\
& =\frac{\varepsilon \operatorname{Re}\left(\mathrm{A}_{0}\right)+\mathrm{i} \operatorname{Im}\left(\mathrm{A}_{0}\right)}{i \varepsilon \operatorname{Im}\left(\mathrm{A}_{0}\right)+\operatorname{Re}\left(\mathrm{A}_{0}\right)} \\
& =\frac{\varepsilon+i \frac{\operatorname{Im}\left(\mathrm{A}_{0}\right)}{\operatorname{Re}\left(\mathrm{A}_{0}\right)}}{1+i \varepsilon \frac{\operatorname{Im}\left(\mathrm{A}_{0}\right)}{\operatorname{Re}\left(\mathrm{A}_{0}\right)}}, \\
\varepsilon_{2} & =\frac{1}{\sqrt{2}} \frac{\left\langle\pi \pi, I=2|\widehat{\mathcal{T}}| \mathrm{K}^{0}\right\rangle\left\langle\mathrm{K}^{0} \mid K_{L}^{0}\right\rangle+\left\langle\pi \pi, I=2|\widehat{\mathcal{T}}| \overline{\mathrm{K}}^{0}\right\rangle\left\langle\overline{\mathrm{K}}^{0} \mid K_{L}^{0}\right\rangle}{\left\langle\pi \pi, I=0|\widehat{\mathcal{T}}| \mathrm{K}^{0}\right\rangle\left\langle\mathrm{K}^{0} \mid K_{S}^{0}\right\rangle+\left\langle\pi \pi, I=0|\widehat{\mathcal{T}}| \overline{\mathrm{K}}^{0}\right\rangle\left\langle\overline{\mathrm{K}}^{0} \mid K_{S}^{0}\right\rangle} \\
& =\frac{1}{\sqrt{2}} e^{i\left(\delta_{2}-\delta_{0}\right)} \frac{A_{2}-A_{2}^{*}+\varepsilon\left(A_{2}+A_{2}^{*}\right)}{A_{0}-A_{0}^{*}+\varepsilon\left(A_{0}+A_{0}^{*}\right)} \\
& =\frac{1}{\sqrt{2}} e^{i\left(\delta_{2}-\delta_{0}\right)} \frac{\varepsilon \operatorname{Re}\left(\mathrm{A}_{2}\right)+\mathrm{i} \operatorname{Im}\left(\mathrm{A}_{2}\right)}{\operatorname{Re}\left(\mathrm{A}_{0}\right)+\mathrm{i} \varepsilon \operatorname{Im}\left(\mathrm{A}_{0}\right)} \\
& =\varepsilon^{\prime} .
\end{aligned}
$$

In any phase convention where $\operatorname{Im}\left(\mathrm{A}_{0}\right) \ll \operatorname{Re}\left(\mathrm{A}_{0}\right)$, one then has the approximations

$$
\begin{aligned}
\eta_{+-} & =\left|\eta_{+-}\right| e^{i \phi_{+-}} \\
& \simeq \varepsilon+\varepsilon^{\prime}, \\
\eta_{00} & =\left|\eta_{00}\right| e^{i \phi 00} \\
& \simeq \varepsilon-2 \varepsilon^{\prime} .
\end{aligned}
$$

The importance of the definition of $\varepsilon$ and $\varepsilon^{\prime}$ is that the CP violation which occurs due to differing decay amplitudes, so called direct $\mathrm{CP}$ violation, is isolated into $\varepsilon$, while the $\mathrm{CP}$ violation due to mixing is parameterised by $\varepsilon$. In this way, if $\eta_{+-}$is not equal to $\eta_{00}$, then $\mathrm{CP}$ is violated in the decay amplitudes as well as the mixing. The experimental values for the above quantities are:

$$
\begin{aligned}
\left|\eta_{00}\right| & =2.259 \pm 0.023 \times 10^{-3} \\
\phi_{00} & =43.3 \pm 1.3^{o} \\
\left|\eta_{+-}\right| & =2.269 \pm 0.023 \times 10^{-3} \\
\phi_{+-} & =44.3 \pm 1.3^{o} \\
\frac{\varepsilon^{\prime}}{\varepsilon} & = \begin{cases}2.30 \pm 0.65 \times 10^{-3} & \mathrm{NA} 31 \\
7.4 \pm 6.0 \times 10^{-4} & \mathrm{E} 731\end{cases}
\end{aligned}
$$


from the CERN[10] and Fermilab[11] experiments. The current experimental situation regarding $\varepsilon^{\prime}$ does not yet provide unambiguous evidence for direct $\mathrm{CP}$ violation.

\section{4 $C P$ violation and mixing in the $B$ meson system}

\subsubsection{Phenomenology}

The phenomenology described above can be employed in a similar fashion in the $B$ meson system. There is no measurement of the lifetime difference, but since it must arise from decay channels common to both the $\mathrm{B}^{0}$ and the $\overline{\mathrm{B}}^{0}$ and these are generally $\mathcal{O}\left(10^{-3}\right)$ or less, the difference is thought to be $\mathcal{O}\left(10^{-2}\right)$. In any event, $\Delta \Gamma_{B_{d}} \ll \Gamma_{B_{d}}$ has been shown to be a safe assumption[12]. The experimental result[9]

$$
\frac{\Delta m_{B_{d}}}{\Gamma_{B_{d}}}=0.73 \pm 0.05
$$

then gives us $\Delta m_{B_{d}} \gg \Delta \Gamma_{B_{d}}$. This is reflected in the naming of the mass eigenstates, now termed $B_{L}^{0}$ and $B_{H}^{0}$, for light and heavy:

$$
\begin{aligned}
\left|B_{L}^{0}\right\rangle & =p\left|\mathrm{~B}^{0}\right\rangle+q\left|\overline{\mathrm{B}}^{0}\right\rangle \\
\left|B_{H}^{0}\right\rangle & =p\left|\mathrm{~B}^{0}\right\rangle-q\left|\overline{\mathrm{B}}^{0}\right\rangle .
\end{aligned}
$$

The smallness of $\Delta \Gamma_{B_{d}}$ also allows us to make simplifications in the formulae describing the time development of the physical states, so that equation 1.8 becomes:

$$
\begin{aligned}
& f_{+}(t)=\exp \left\{-\left(i m+\frac{\Gamma}{2}\right)\right\} \cos \left(\frac{\Delta m_{B_{d}} t}{2}\right) \\
& f_{-}(t)=i \exp \left\{-\left(i m+\frac{\Gamma}{2}\right)\right\} \sin \left(\frac{\Delta m_{B_{d}} t}{2}\right)
\end{aligned}
$$

The mixing of pure states in the $B$ meson system was first observed unambiguously by the ARGUS collaboration [13].

\subsection{2 $C P$ violation in $B$ decays}

\subsubsection{Direct $C P$ violation}

Direct $C P$ violation is a manifestation of differing decay amplitudes for a particle/anti-particle pair. In the Standard Model Lagrangian, the fields are formulated in such a way that their $C P$ 
transformations are given by[14]

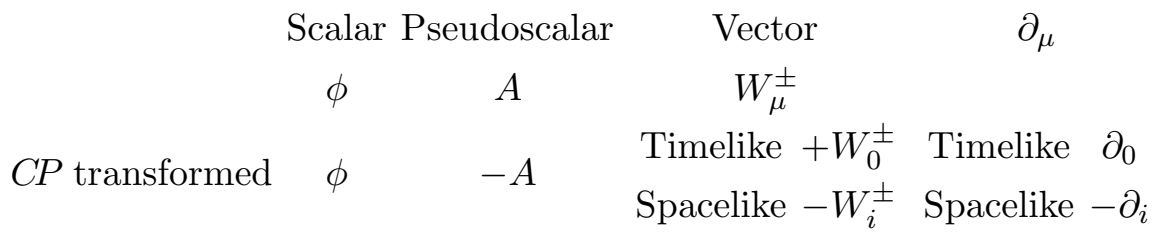

and that the effect of the $C P$ transformation on the whole Lagrangian is to transform it to its Hermitian conjugate. However, there will in general be complex coefficients in front of the terms in the Lagrangian and these will not transform. This is the origin of the $\mathrm{CP}$ non-equivalence of the transformed fields. In fact, ignoring the strong $\mathrm{CP}$ violating terms, which are known to be vanishingly small[15], such terms are confined to the weak sector, specifically in the CKM mechanism described in section 1.5.1.

Considering a decay of a $B$ meson to some final state $f$, the amplitude of this decay may be written

$$
\begin{aligned}
A & =\left\langle f|\hat{H}| \mathrm{B}^{0}\right\rangle=\sum_{i} A_{i} e^{i \delta_{i}} e^{i \phi_{i}} \\
\bar{A} & =\left\langle\bar{f}|\hat{H}| \overline{\mathrm{B}}^{0}\right\rangle=e^{\xi_{C P}} \bar{A}_{i} e^{i \delta_{i}} e^{-i \phi_{i}}
\end{aligned}
$$

where $e^{\xi_{C P}}$ is a $C P$ phase, $\delta_{i}$ denotes the strong phase, unchanged by the $C P$ transformation and $\phi_{i}$ the weak phase thus conjugated. The ratio $|A / \bar{A}|$ being different from unity would imply direct $C P$ violation. It can be seen that at least two different strong and weak phases must exist for the same transition $\mathrm{B}^{0} \rightarrow f$ for this to occur. For the simplest case where two such phases exist, the rates may be written as

$$
\begin{aligned}
& \Gamma_{1}=\left|A_{1} e^{i\left(\delta_{1}+\phi_{1}\right)}+A_{2} e^{i\left(\delta_{2}+\phi_{2}\right)}\right|^{2} \\
& \Gamma_{2}=\left|A_{1} e^{i\left(\delta_{1}-\phi_{1}\right)}+A_{2} e^{i\left(\delta_{2}-\phi_{2}\right)}\right|^{2}
\end{aligned}
$$

giving

$$
\Gamma_{1}-\Gamma_{2} \propto 2 A_{1} A_{2} \sin \left(\phi_{1}-\phi_{2}\right) \sin \left(\delta_{1}-\delta_{2}\right)
$$

from which it may be seen that both the strong phase difference and the weak phase difference must be non-zero for direct $C P$ to be observed. In the decays of charged $B$ mesons, this is the only way in which $C P$ violation can be observed. To date, however, no such observation has been made. The situation with $\varepsilon^{\prime} / \varepsilon$ in the $K$ meson system is inconclusive as indicated earlier, but a non-zero value would be evidence for direct $C P$ violation.

\subsubsection{CP violation in mixing}

$C P$ violation in mixing amounts to the statement that in equations 1.45 and $1.46,|q / p| \neq 1$. In order to study this, it is necessary to find a decay in which one of the amplitudes $\mathrm{B}^{0} \rightarrow f$ and $\overline{\mathrm{B}}^{0} \rightarrow f$ is zero to avoid the possibility of $C P$ violation by interference between decays (see section 1.4.2.3). An example is the semi-leptonic decay $\mathrm{B}^{0} \rightarrow l^{-} \bar{\nu}_{l} X$, for which $\Gamma\left(\overline{\mathrm{B}}^{0} \rightarrow l^{-} \bar{\nu}_{l} X\right)$ 
and $\Gamma\left(\mathrm{B}^{0} \rightarrow l^{+} \nu_{l} X\right)$ are both zero. Unlike in the kaon system where the large difference in lifetimes allows preparation of a reasonably pure sample of $K_{L}^{0}$, separation of the mass eigenstates in the $B$ meson system is not possible. A measurement of the time dependent asymmetry

$$
a=\frac{\Gamma\left(\overline{\mathrm{B}}^{0}(t) \rightarrow l^{+} \nu_{l} X\right)-\Gamma\left(\mathrm{B}^{0}(t) \rightarrow l^{-} \bar{\nu}_{l} X\right)}{\Gamma\left(\overline{\mathrm{B}}^{0}(t) \rightarrow l^{+} \nu_{l} X\right)+\Gamma\left(\mathrm{B}^{0}(t) \rightarrow l^{-} \bar{\nu}_{l} X\right)}
$$

would provide evidence for $C P$ violation in mixing, but unfortunately such effects are thought to be small $\left(\mathcal{O}\left(10^{-2}\right)\right)[16]$.

\subsubsection{CP violation in the interference between decays}

If one is interested in the decay of a $\mathrm{B}^{0}$ to some $\mathrm{CP}$ eigenstate $\xi_{C P}$ via some Hamiltonian $\widehat{H}$, then one can define

$$
\begin{aligned}
& A=\left\langle\xi_{C P}|\hat{H}| \mathrm{B}^{0}\right\rangle \\
& \bar{A}=\left\langle\xi_{C P}|\hat{H}| \overline{\mathrm{B}}^{0}\right\rangle
\end{aligned}
$$

and

$$
\lambda=\frac{q}{p} \frac{\bar{A}}{A}
$$

The time dependent amplitudes are calculated using

$$
\begin{aligned}
& \left\langle\xi_{C P}|\hat{H}| \mathrm{B}^{0}(t)\right\rangle=A\left(f_{+}(t)+\lambda f_{-}(t)\right) \\
& \left\langle\xi_{C P}|\hat{H}| \overline{\mathrm{B}}^{0}(t)\right\rangle=A \frac{p}{q}\left(f_{-}(t)+\lambda f_{+}(t)\right)
\end{aligned}
$$

giving

$$
\begin{aligned}
\Gamma\left(\mathrm{B}^{0}(t) \rightarrow \xi_{C P}\right)= & \left|\left\langle\xi_{C P}|\widehat{H}| \mathrm{B}^{0}(t)\right\rangle\right|^{2} \\
= & |A|^{2}\left[e^{-\gamma t} \cos ^{2}\left(\frac{\Delta M t}{2}\right)+e^{-\gamma t}|\lambda|^{2} \sin ^{2}\left(\frac{\Delta M t}{2}\right)\right. \\
& \left.+e^{-\gamma t} \lambda \cos ^{*}\left(\frac{\Delta M t}{2}\right) \sin \left(\frac{\Delta M t}{2}\right)-e^{-\gamma t} \lambda^{*} \cos \left(\frac{\Delta M t}{2}\right) \sin ^{*}\left(\frac{\Delta M t}{2}\right)\right], \\
= & |A|^{2} e^{-\gamma t}\left[\frac{1+|\lambda|^{2}}{2}+\frac{1-|\lambda|^{2}}{2} \cos (\Delta M t)-\operatorname{Im}(\lambda) \sin (\Delta \mathrm{Mt})\right] .
\end{aligned}
$$

Similarly

$$
\Gamma\left(\overline{\mathrm{B}}^{0}(t) \rightarrow \xi_{C P}\right)=|A|^{2} e^{-\gamma t}\left[\frac{1+|\lambda|^{2}}{2}-\frac{1-|\lambda|^{2}}{2} \cos (\Delta M t)+\operatorname{Im}(\lambda) \sin (\Delta \mathrm{Mt})\right] .
$$

The key point is that these amplitudes have two contributions, one from the direct decay of the $\mathrm{B}^{0}$ into the state $\xi_{C P}$ and one from the mixing of the $\mathrm{B}^{0}$ into a $\overline{\mathrm{B}}^{0}$ which then decays into $\xi_{C P}$. 

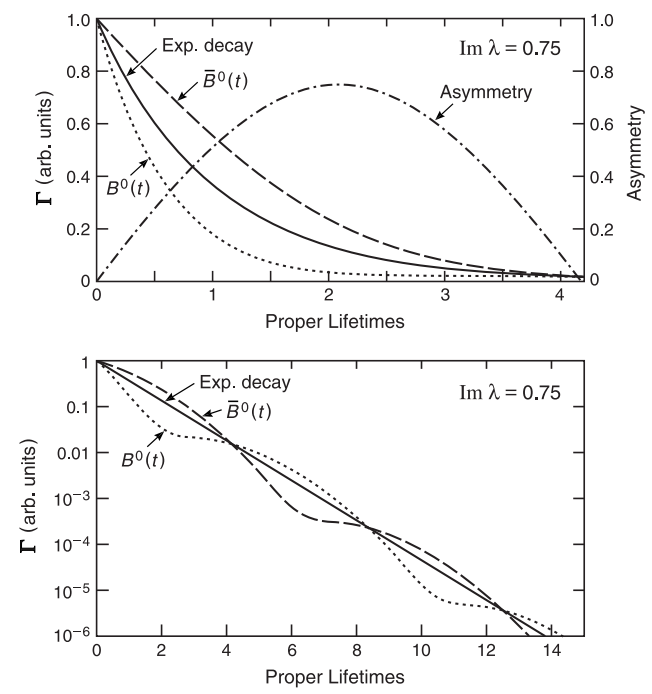

Figure 1-1. $\quad B$ meson decay rates as a function of lifetime for a $C P$ asymmetry of $\operatorname{Im}(\lambda)=0.75$.

If no $C P$ violation were observed, the two decay rates would follow the exponential (solid line).

This gives this form of $\mathrm{CP}$ violation its full title: " $\mathrm{CP}$ violation in the interference between decays with and without mixing." The condition for this to occur is that $\lambda$ be different from one. This will be true automatically if direct CP violation occurs in this decay, and also if CP violation in mixing alone occurs, but is a weaker condition than either of these two. Typical decay curves in the presence of $C P$ violation are shown in figure 1-1[17]. It is useful to factor out the amplitude $A$ and the decay constant $\gamma$, by forming the ratio

$$
\begin{aligned}
a_{\xi_{C P}}(t) & =\frac{\Gamma\left(\mathrm{B}^{0}(t) \rightarrow \xi_{C P}\right)-\Gamma\left(\overline{\mathrm{B}}^{0}(t) \rightarrow \xi_{C P}\right)}{\Gamma\left(\mathrm{B}^{0}(t) \rightarrow \xi_{C P}\right)+\Gamma\left(\overline{\mathrm{B}}^{0}(t) \rightarrow \xi_{C P}\right)} \\
& =\frac{\left(1-|\lambda|^{2}\right) \cos (\Delta M t)-2 \operatorname{Im}(\lambda) \sin (\Delta \mathrm{Mt})}{1+|\lambda|^{2}}
\end{aligned}
$$

which in the absence of direct $C P$ violation and $C P$ violation in mixing $(|\lambda|=1)$ becomes

$$
a_{\xi_{C P}}(t)=-\operatorname{Im}(\lambda) \sin (\Delta \mathrm{Mt}) .
$$

\subsubsection{Measuring $a_{\xi_{C P}}(t)$.}

In the above equations, $t$ denotes the time elapsed since the flavour of the $b$ quark can be determined. At the $\Upsilon(4 \mathrm{~S})$ the $\mathrm{B}^{0}$ and $\overline{\mathrm{B}}^{0}$ are in produced in a coherent state, and this allows the determination of the flavour of the $b$ quark by the study of the decay of the $B$ meson not decaying 
to a $C P$ eigenstate - the so-called "tag" $B$. In this case $t$ is the time difference between the two decays, which can be negative.

If the $\Upsilon(4 \mathrm{~S})$ decays at rest, the momentum of each $\mathrm{B}^{0}$ is $340 \mathrm{MeV}$, which results in a typical flight length difference of approximately $30 \mu \mathrm{m}$. Such a separation is not observable with current vertex detector technology, and it is for this reason that an asymmetric collider is preferred. A boosted $\Upsilon(4 \mathrm{~S})$ giving the daughter $B$ mesons a $\beta \gamma$ in the region of 10 times that of a stationary $\Upsilon(4 \mathrm{~S})$ is chosen. The parameters chosen at the PEP-II storage ring to achieve this are described in chapter 2.

\subsection{Quark mixing and the CKM matrix}

\subsubsection{The CKM description of $C P$ violation}

The electro-weak Lagrangian, before symmetry breaking, may be written as ([18] chapters 11 and 12 )

$$
-\mathcal{L}_{W}=\frac{g}{\sqrt{2}}\left(\bar{u}_{L}^{I} \bar{c}_{L}^{I} \bar{t}_{L}^{I}\right) \gamma^{\mu}\left(\begin{array}{c}
\bar{d}_{L}^{I} \\
\bar{s}_{L}^{I} \\
\bar{b}_{L}^{I}
\end{array}\right) W_{\mu}^{+}+H . c .
$$

where $I$ denotes the interaction states, $L$ denotes the left handed projections of the fermion fields $u$, and $W^{+}$denotes the charge raising electroweak-field operator. The weak interaction permits fields to connect with one another, and so the resulting observed mass eigenstates are mixed states of the original quarks. Denoting the transformations by which the original mass matrix is diagonalised by $V_{i L}$ and $V_{i R}$, for the left and right fermionic fields $i$ respectively, one has that

$$
\begin{aligned}
V_{d L} M_{d} V_{d R}^{\dagger} & =M_{d}^{d i a g} \\
V_{u L} M_{u} V_{u R}^{\dagger} & =M_{u}^{d i a g}
\end{aligned}
$$

and, substituting into the Lagrangian,

$$
-\mathcal{L}_{W}=\frac{g}{\sqrt{2}}\left(\bar{u}_{L} \bar{c}_{L} \bar{t}_{L}\right) \gamma^{\mu} V_{u L} V_{d L}^{\dagger}\left(\begin{array}{c}
\bar{d}_{L} \\
\bar{s}_{L} \\
\bar{b}_{L}
\end{array}\right) W_{\mu}^{+}+H . c
$$

One may define the matrix $V$ such that

$$
\begin{aligned}
V & =V_{u L} V_{d L}^{\dagger} \\
& =\left(\begin{array}{lll}
V_{u d} & V_{u s} & V_{u b} \\
V_{c d} & V_{c s} & V_{c b} \\
V_{t d} & V_{t s} & V_{t b}
\end{array}\right) .
\end{aligned}
$$


This is the CKM matrix [19]. A general $n \times n$ complex matrix, has $2 n^{2}$ parameters. The CKM matrix must be unitary (to preserve probability), and this gives $n^{2}$ constraints. Of the remaining parameters, 3 , being the number of independent rotations in 3 dimensions, can be written as angles, while 6 are phases. One may redefine the quark fields thus:

$$
\begin{aligned}
& \left(\begin{array}{l}
\bar{u}^{\prime} L \\
{\overline{c^{\prime}}}_{L} \\
{\overline{t^{\prime}}}_{L}
\end{array}\right)=P_{u}\left(\begin{array}{l}
\bar{u}_{L} \\
\bar{c}_{L} \\
\bar{t}_{L}
\end{array}\right) \\
& \left(\begin{array}{c}
\bar{d}^{\prime}{ }_{L} \\
{\overline{s^{\prime}}}_{L} \\
{\overline{b^{\prime}}}_{L}
\end{array}\right)=P_{d}\left(\begin{array}{l}
\bar{d}_{L} \\
\bar{s}_{L} \\
\bar{b}_{L}
\end{array}\right)
\end{aligned}
$$

where $P_{u}$ and $P_{d}$ are diagonal matrices consisting exclusively of phases. The result of this transformation is that $V$ is redefined thus

$$
V^{\prime}=\left(\begin{array}{lll}
e^{-i\left(\phi_{1}-\xi_{1}\right)} V_{u d} & e^{-i\left(\phi_{1}-\xi_{2}\right)} V_{u s} & e^{-i\left(\phi_{1}-\xi_{3}\right)} V_{u b} \\
e^{-i\left(\phi_{2}-\xi_{1}\right)} V_{c d} & e^{-i\left(\phi_{2}-\xi_{2}\right)} V_{c s} & e^{-i\left(\phi_{2}-\xi_{3}\right)} V_{c b} \\
e^{-i\left(\phi_{3}-\xi_{1}\right)} V_{t d} & e^{-i\left(\phi_{3}-\xi_{2}\right)} V_{t s} & e^{-i\left(\phi_{3}-\xi_{3}\right)} V_{t b}
\end{array}\right)
$$

where $\phi_{i}$ is phase of $\left(P_{u}\right)_{i i}$ and $\xi_{i}$ that of $\left(P_{d}\right)_{i i}$. It is clear from this expression that only 5 independent phase differences occur, and thus the 6th cannot be rotated away. It is this complex phase, $\delta_{K M}$, which leads to $C P$ violation, and which led to the postulation of the third quark generation. This was noted by Jarlskog[20], who defined the quantity

$$
J=\left|\operatorname{Im}\left(V_{i j} V_{k l} V_{i l}^{*} V_{k j}^{*}\right)\right|, i \neq k, j \neq l
$$

which is invariant under the phase transformations just described, and a non zero value for which is a necessary and sufficient condition for $C P$ violation, ignoring the possibility of mass degeneracy amongst the quarks. In the standard parameterisation of the CKM matrix[9]:

$$
V=\left(\begin{array}{ccc}
c_{12} c_{13} & s_{12} c_{13} & s_{13} e^{-i \delta_{K M}} \\
-s_{12} c_{23}-c_{12} s_{23} s_{13} e^{-i \delta_{K M}} & c_{12} c_{23}-s_{12} s_{23} s_{13} e^{-i \delta_{K M}} & s_{23} c_{13} \\
s_{12} c_{23}-c_{12} c_{23} s_{13} e^{-i \delta_{K M}} & -c_{12} s_{23}-s_{12} c_{23} s_{13} e^{-i \delta_{K M}} & c_{23} c_{13}
\end{array}\right)
$$

where $\theta_{i j}$ denotes the mixing angle between the $i^{\text {th }}$ and $j^{\text {th }}$ generations (in particular $\theta_{12}$ denoting the Cabbibo angle $\theta_{c}$ [21]), and $s_{i j}=\sin \theta_{i j}, c_{i j}=\cos \theta_{i j}$. The expression for $J$ becomes

$$
J=\left|s_{13} s_{23} s_{12} c_{13}^{2} c_{23} c_{12} \sin \delta_{K M}\right|
$$

one has that $J \leq 10^{-4}[16]$, i.e. that $C P$ violation is expected to be a small effect.

Noting that $c_{13} \simeq 1$ and denoting the Cabbibo angle as $\lambda$, the matrix was parameterised by Wolfenstein [22] thus:

$$
V=\left(\begin{array}{ccc}
\cos \lambda & \sin \lambda & A \lambda^{3}(\rho-i \eta) \\
-\sin \lambda & \cos \lambda & A \lambda^{2} \\
A \lambda^{3}(1-\rho-i \eta) & -A \lambda^{2} & 1
\end{array}\right)+\mathcal{O}\left(\lambda^{4}\right)
$$


Noting that experimentally, $\sin \theta_{c} \simeq 0.22$, one may use a small angle approximation to recover the "standard" Wolfenstein approximation

$$
V=\left(\begin{array}{ccc}
1-\lambda^{2} / 2 & \lambda & A \lambda^{3}(\rho-i \eta) \\
-\lambda & 1-\lambda^{2} / 2 & A \lambda^{2} \\
A \lambda^{3}(1-\rho-i \eta) & -A \lambda^{2} & 1
\end{array}\right)+\mathcal{O}\left(\lambda^{4}\right)
$$

Unitarity imposes the condition

$$
V_{u d} V_{u b}^{*}+V_{c d} V_{c b}^{*}+V_{t d} V_{t b}^{*}=0 .
$$

This equation is equivalent to one of the so-called unitarity triangles in the complex plane, shown in fig 1-2(a). The area of all such triangles is $1 / 2 J$ - i.e. invariant. Substituting the Wolfenstein parameters for the matrix elements, and dividing throughout by $V_{c d} V_{c b}^{*}$, one arrives at a triangle whose base is unity and whose vertex is given by $\bar{\rho}, \bar{\eta}$, where

$$
\begin{aligned}
& \bar{\rho}=\left(1-\frac{\lambda^{2}}{2}\right) \rho \\
& \bar{\eta}=\left(1-\frac{\lambda^{2}}{2}\right) \eta
\end{aligned}
$$

This is shown in figure 1-2(b).

How can the formalism in the previous section be related to the CKM elements? Recalling the definition of $\lambda$ used in equation 1.52 ,

$$
\lambda=\frac{q}{p} \frac{\bar{A}}{A}
$$

(it should be noted that this is not the Wolfenstein $\lambda$ ), one is interested in the value of $\lambda$ when the decay mode in question is to a CP eigenstate. In this case the dominant contributions to the matrix elements $A$ and $\bar{A}$ are from tree diagrams [23, 24, 25], such as the one shown in fig.2(b) and so one may write (for the case of the $B_{d}^{0}$ )

$$
\frac{\bar{A}}{A}=\frac{V_{i b} V_{i d}^{*}}{V_{i b}^{*} V_{i d}}
$$

Furthermore, it is clear from this equation that if one particular CKM contribution dominates, then we have that

$$
\left|\frac{\bar{A}}{A}\right|=1 \text {. }
$$

From the eigenvalue equations for $q$ and $p$, one can write

$$
\left(\frac{q}{p}\right)^{2}=\frac{m_{21}-i \Gamma_{21} / 2}{m_{12}-i \Gamma_{12} / 2} .
$$




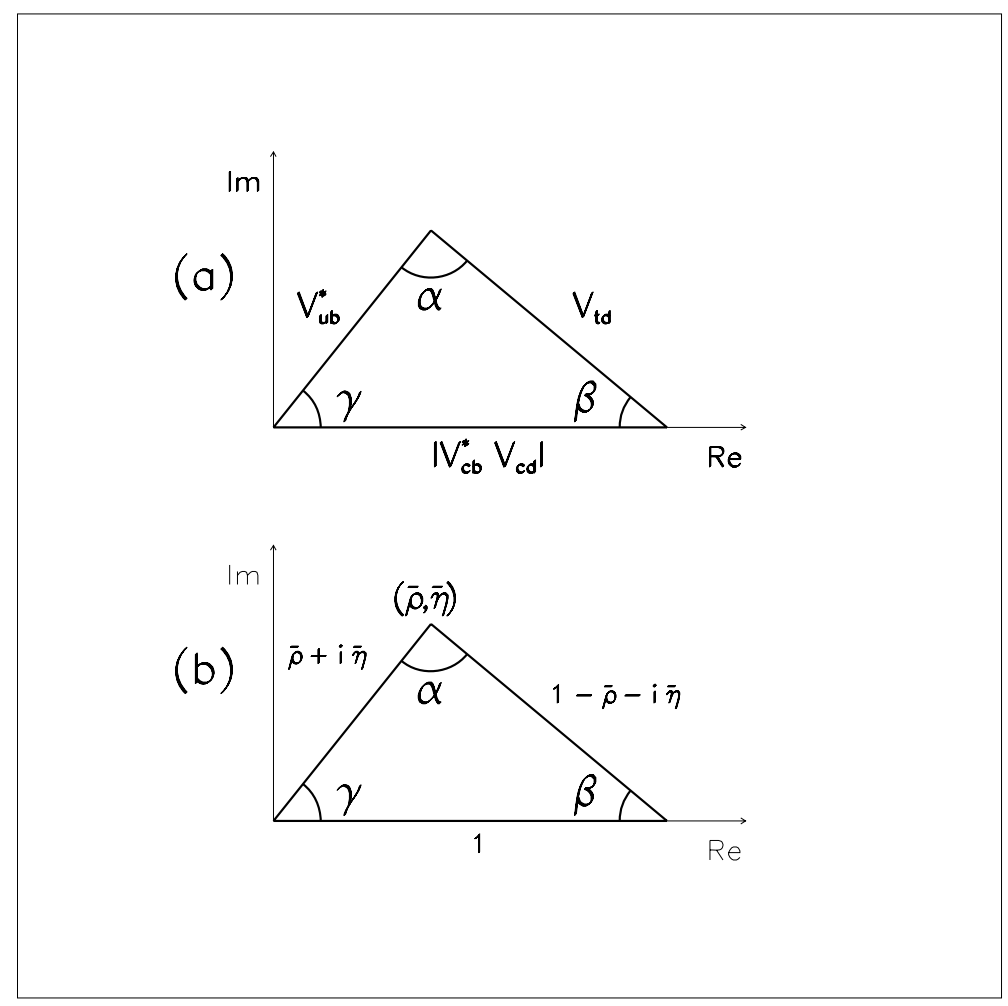

Figure 1-2. Unitarity triangles in the complex plane. Figure (a) depicts the triangle relating to possible $C P$ violation in $B$ physics. Figure (b) is the same triangle in the Wolfenstein parameterisation.

Under CPT invariance, the elements of the mass matrix must obey $M_{21}=M_{12}^{*}$ [7], and so one has that

$$
\frac{q}{p}=\left(\frac{M_{12}^{*}}{M_{12}}\right)^{1 / 2}
$$

The mixing element $M_{12}$ comes from diagrams such as that shown in fig.2(a). One consequently has the relation $M_{12} \propto\left(V_{t b} V_{t d}^{*}\right)^{2}$ and therefore that

$$
\frac{q}{p}=\frac{V_{t b} V_{t d}^{*}}{V_{t d} V_{t b}^{*}}
$$

It is usual to express these quantities thus:

$$
\begin{aligned}
& \frac{q}{p}=e^{-2 i \phi_{M}} \\
& \frac{\bar{A}}{A}=e^{-2 i \phi_{D}} .
\end{aligned}
$$

such that

$$
\operatorname{Im}(\lambda)=-2 \sin 2\left(\phi_{\mathrm{M}}+\phi_{\mathrm{D}}\right)
$$




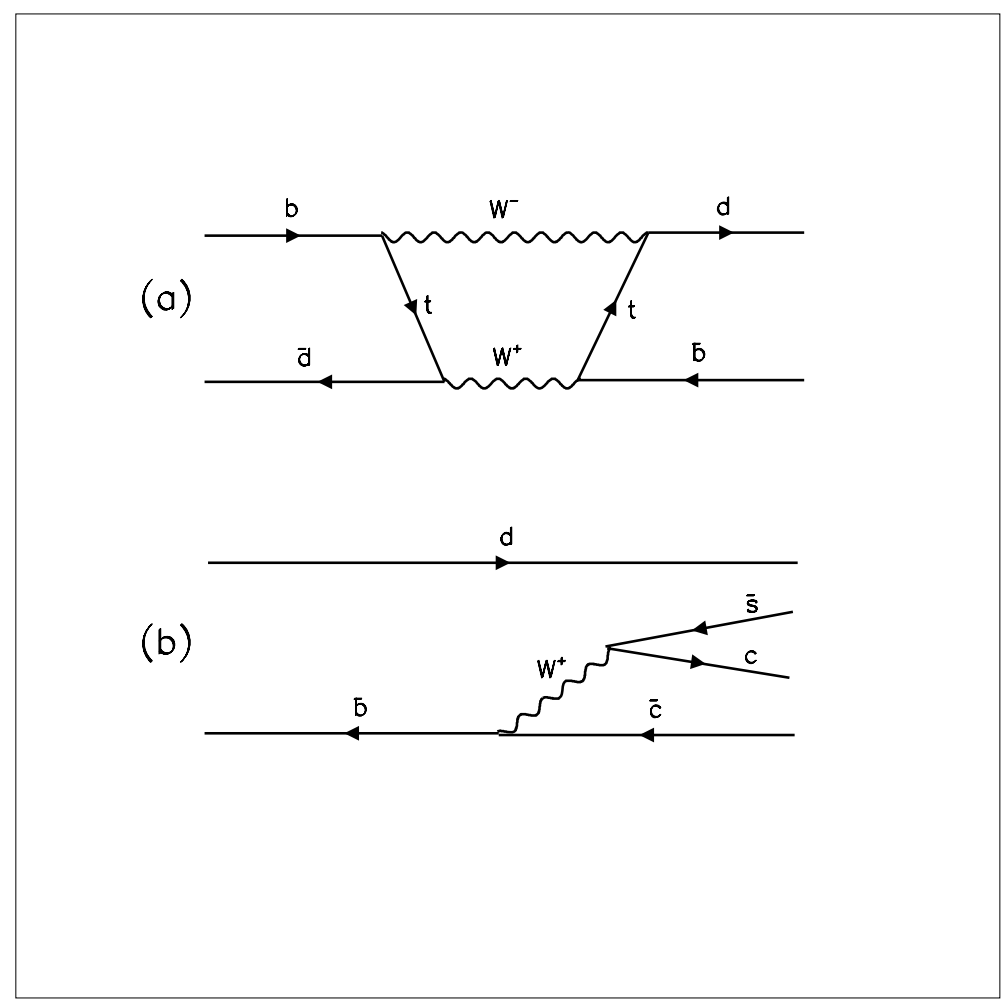

Figure 1-3. Figure (a) depicts a typical process for $\mathrm{B}^{0}$ mixing. Figure (b) is a typical decay, $\overline{\mathrm{B}}^{0} \rightarrow J / \psi K_{S}^{0}$

i.e. that

$$
\operatorname{Im}(\lambda)=\sin \left(\arg \frac{\mathrm{V}_{\mathrm{tb}} \mathrm{V}_{\mathrm{td}}^{*}}{\mathrm{~V}_{\mathrm{td}} \mathrm{V}_{\mathrm{tb}}^{*}} \frac{\mathrm{V}_{\mathrm{ib}} \mathrm{V}_{\mathrm{id}}^{*}}{\mathrm{~V}_{\mathrm{ib}}^{*} \mathrm{~V}_{\mathrm{id}}}\right) .
$$

The angles in the unitarity triangle are defined as

$$
\begin{gathered}
\alpha=\arg \left(-\frac{V_{t d} V_{t b}^{*}}{V_{u d} V_{u b}^{*}}\right) \\
\beta=\arg \left(-\frac{V_{c d} V_{c b}^{*}}{V_{t d} V_{t b}^{*}}\right) \\
\gamma=\arg \left(-\frac{V_{u d} V_{u b}^{*}}{V_{c d} V_{c b}^{*}}\right) .
\end{gathered}
$$

These angles may be determined by measuring appropriate channels in neutral $B$ decays [26, 27]. Examples are the following:

$\sin 2 \beta$ from $B_{d}^{0} \rightarrow J / \psi K_{S}^{0}$. In this channel, the $\mathrm{K}^{0}$ mixing must be taken in to account. The correction is that

$$
\lambda=\left(\frac{q}{p}\right)\left(\frac{\bar{A}}{A}\right)\left(\frac{q}{p}\right)_{K}
$$


where

$$
\left(\frac{q}{p}\right)_{K}=\frac{V_{c s} V_{c d}^{*}}{V_{c s}^{*} V_{c d}}
$$

so then

$$
\begin{aligned}
\lambda_{B_{d}^{0} \rightarrow J / \psi K_{S}^{0}} & =\frac{V_{t d} V_{t b}^{*}}{V_{t d}^{*} V_{t b}} \frac{V_{c b} V_{c s}^{*}}{V_{c b}^{*} V_{c s}} \frac{V_{c s} V_{c d}^{*}}{V_{c s}^{*} V_{c d}} \\
& =\frac{V_{t d} V_{t b}^{*}}{V_{t b} V_{t d}^{*}} \frac{V_{c d}^{*} V_{c b}}{V_{c b}^{*} V_{c d}} \\
\Rightarrow \operatorname{Im}(\lambda) & =-\sin (2 \beta) .
\end{aligned}
$$

$\sin 2 \alpha$ from $B_{d}^{0} \rightarrow \pi^{+} \pi^{-}$. Here the expression for $\lambda$ is

$$
\begin{aligned}
\lambda_{B_{d}^{0} \rightarrow \pi^{+} \pi^{-}} & =\frac{V_{t d} V_{t b}^{*}}{V_{t d}^{*} V_{t b}} \frac{V_{u b} V_{u d}^{*}}{V_{u b}^{*} V_{u d}} \\
\Rightarrow \operatorname{Im}(\lambda) & =\sin (2(\pi-\beta-\gamma) \\
& =\sin (2 \alpha) .
\end{aligned}
$$

$\sin 2 \gamma$ from $B_{s}^{0} \rightarrow \rho K_{S}^{0}$. Again we have $\mathrm{K}^{0}$ mixing, and thus

$$
\lambda_{B_{s}^{0} \rightarrow \rho K_{S}^{0}}=\frac{V_{t s} V_{t b}^{*}}{V_{t s}^{*} V_{t b}} \frac{V_{u b} V_{u d}^{*}}{V_{u b}^{*} V_{u d}} \frac{V_{c s} V_{c d}^{*}}{V_{c s}^{*} V_{c d}}
$$

From the Wolfenstein parameterisation, one may note that $V_{t s} \simeq V_{c b}$ and that $V_{t b} \simeq V_{c s} \simeq 1$ and so

$$
\begin{aligned}
\lambda_{B_{s}^{0} \rightarrow \rho K_{S}^{0}} & =\frac{V_{c b} V_{u d}^{*} V_{u b} V_{c d}^{*}}{V_{u d} V_{c b}^{*} V_{c d} V_{u b}^{*}} \\
\Rightarrow \operatorname{Im}(\lambda) & =-\sin (2 \gamma) .
\end{aligned}
$$

In the case where there is no single dominant CKM contribution the situation is more complicated. Under these circumstances, $|\lambda| \neq 1$, and the asymmetry has terms in both $\sin \Delta m t$ and $\cos \Delta m t$. The condition $|\lambda| \neq 1$ is guaranteed if the decay amplitude ratio $|A / \bar{A}|$ is not unity.

A general amplitude $A$ for a $b \rightarrow q \bar{q} x$ decay may be written

$$
A_{q \bar{q} x}=V_{t b} V_{t x}^{*} P_{x}^{t}+V_{c b} V_{c x}^{*}\left(T_{c \bar{c} x} \delta_{q c}+P_{x}^{c}\right)+V_{u b} V_{u x}^{*}\left(T_{u \bar{u} x} \delta_{q u}+P_{x}^{u}\right) .
$$

Where $V$ are the CKM elements, and $P$ and $T$ denote the matrix elements from penguin and tree diagrams respectively. For each $q$ and $x$, a unitarity relation may be invoked to express one of these three quantities in terms of the other two. For the $B \rightarrow c \bar{c} s$ decay, it is convenient to express the amplitude thus:

$$
A_{c \bar{c} s}=V_{c b} V_{c s}^{*}\left(T_{c \bar{c} s}+P_{s}^{c}-P_{s}^{t}\right)+V_{u b} V_{u s}^{*}\left(P_{s}^{u}-P_{s}^{t}\right) .
$$


For this decay, the term $V_{c b} V_{c s}^{*} \gg V_{u b} V_{u s}^{*}$ and the second term may be safely neglected. The penguin contribution to the decay therefore has the same phase as the tree diagram contribution, and so decays of this type present a clean method of measuring the angle $\beta$.

The situation for the decay $b \rightarrow u \bar{u} d$, measuring the angle $\alpha$ in decays such as $\mathrm{B}^{0} \rightarrow \pi^{+} \pi^{-}$, is somewhat different. The relevant expression for the amplitude, this time keeping terms in $V_{u b} V_{u d}^{*}$ as this occurs in $B$ mixing expressions, is

$$
A_{c \bar{c} s}=V_{u b} V_{u d}^{*}\left(T_{u \bar{u} d}-P_{s d}^{u}-P_{d}^{t}\right)+V_{t b} V_{t d}^{*}\left(P_{d}^{t}-P_{d}^{c}\right) .
$$

The CKM coefficients for both these terms have comparable magnitude. Further, recent results from CLEO[28] indicate that enhancement of the penguin diagrams may result in some cancelation of their OZI suppression, resulting in as much as a $10 \%$ contamination. For such a case, specific analysis techniques must be employed to separate the two contributions [29] and consequently extract the contribution measuring $\alpha$.

\subsection{CKM Status and Current Constraints}

Existing measurements allow a considerable amount of information to be extracted on the size of the angles in the unitarity triangle without needing a time dependent asymmetry measurement. A full review of the CKM elements can be found in [9], and a recent review of CKM elements and other parameters with particular relevance to $C P$ violation in the $B$ system can be found in [30]. The key results are summarised here.

The sides of the triangle in the lower half of figure 1-2 are given by

$$
R_{b}=\sqrt{\bar{\rho}^{2}+\bar{\eta}^{2}}=\frac{1-\lambda^{2} / 2}{\lambda}\left|\frac{V_{u b}}{V_{c b}}\right|, R_{t}=\sqrt{(1-\bar{\rho})^{2}+\bar{\eta}^{2}}=\frac{1}{\lambda}\left|\frac{V_{t d}}{V_{c b}}\right|
$$

The relevant CKM parameters are therefore $V_{c b}, V_{u b}$ and $V_{t d}$ and $\lambda$, which to a high degree of accuracy can be equated to $V_{u s}$. The current values are given by[9]

$$
\begin{aligned}
\left|V_{c b}\right| & =0.0395 \pm 0.0017 \\
\left|V_{u b}\right| & =3.3 \pm 0.8 \times 10^{-3} \\
\left|V_{u s}\right| & =0.2196 \pm 0.0023 \\
\left|V_{t d}\right| & \simeq\left|V_{t b}^{*} V_{t d}\right|=0.0084 \pm 0.0018
\end{aligned}
$$

$V_{c b}$ can be derived from the exclusive semi-leptonic decay $B \rightarrow D^{*} l \nu_{l}$, or from inclusive semileptonic $B$ decays. $V_{u b}$ has been measured directly in the decays $B \rightarrow \pi l \nu_{l}$ and $B \rightarrow \rho l \nu_{l}$ at CLEO[33]. An indirect determination can be made from the end point of the semi-leptonic decays of $B$ mesons, above the kinematic limit for the $b \rightarrow c l \nu_{l}$ decay. This yields

$$
\left|V_{u b} / V_{c b}\right|=0.08 \pm 0.02 \text {. }
$$


$V_{u s}$ is determined from the decay rates of neutral $K$ mesons to the final state $\pi l \nu$. The product $\left|V_{t b}^{*} V_{t d}\right|$ is determined from $B$ mixing. The rate of oscillation is governed by the mass difference $\Delta m_{B_{d}}$, as seen in equations 1.47 and 1.48. The expression for $\Delta m_{B_{d}}$ gives [34]

$$
\Delta m_{B_{d}} \propto B_{B_{d}} f_{B_{d}}^{2} \eta_{Q C D}\left|V_{t b}^{*} V_{t d}\right|^{2}
$$

where the factor $B_{B_{d}} f_{B_{d}}^{2}$ describes the form factor for the quarks inside the $B$ meson and $\eta_{Q C D}$ gives the QCD correction to the box diagram shown in figure 1-3(a). These have not been measured by experiment and it is the theoretical error on these quantities which dominates the uncertainty on $V_{t d}$. The measurement of $\epsilon$ in the Kaon system also provides limits on the CKM parameters $\rho$ and $\eta$. These can be combined with the limits above to provide information on the allowed regions in the $\rho, \eta$ plane for the vertex of the unitarity triangle. A plot of the currently allowed region can be found in figure 1-4 [31]. A full discussion of these limits and their meaning can be found in [32].

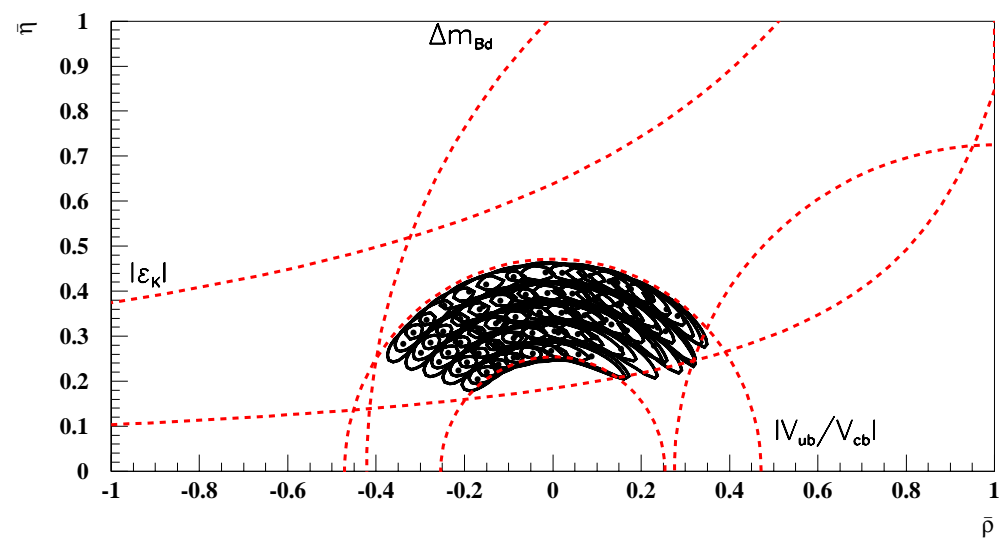

Figure 1-4. Allowed regions in the $\rho, \eta$ plane from current measurements. The dotted lines represent the boundaries of the allowed regions by allowing all parameters to vary. The contours, centred with a dot, represent the 95\% confidence limits region using a given set of theoretical parameters. Limits from $\Delta m_{B_{s}}$ are not included.

\subsection{Chapter Summary}

The phenomenon of neutral meson mixing has been described, and the observation of $C P$ violation in the $K$ meson system outlined. It is concluded that there is no experimental evidence for direct $C P$ violation as yet, instead that it may be explained in terms of mixing phenomena alone. The prospects for the observation of the three types of $C P$ violation in the $B$ meson system have been discussed. The CKM matrix has been introduced, and the expressions for the expected size of $C P$ violating effects have been described in terms of the CKM elements, in particular that the Jarlskog 
invariant

$$
J=\left|\operatorname{Im}\left(V_{i j} V_{k l} V_{i l}^{*} V_{k j}^{*}\right)\right|, i \neq k, j \neq l
$$

which must be non-zero for $C P$ violation to occur, is small. The concept of unitarity triangles has been described and the relation of the angles of the triangle in the $B$ meson system to the CKM elements and observable decays has been given. 


\section{The BaBar experiment and the PEP-II}

\section{storage ring}

\subsection{Introduction}

The attempt to measure $C P$ violation in the $B$ meson system makes considerable demands on the experimental apparatus and the collider. The branching ratios of $B \rightarrow f_{C P}$ where $f_{C P}$ is a $C P$ eigenstate are typically $\mathcal{O}\left(10^{-4}\right)$, giving rise to the need for a very high integrated luminosity, whilst the need to measure precisely the separation of the two $B$ meson decays makes a boost to the $\Upsilon(4 \mathrm{~S})$ system necessary in tandem with precision vertexing. High quality tracking and calorimetry are required, as well as particle identification (in particular good $K / \pi$ separation) necessary to distinguish between important $f_{C P}$ states.

This chapter will begin with a discussion of the asymmetric collider on which the BABAR experiment will be situated, outlining its key components and highlighting the differences between this and conventional colliders. The $B A B A R$ experiment itself will then be described, giving an outline of the tracking systems, particle identification, and calorimetry. The calorimeter will be discussed in more detail in subsequent chapters.

\subsection{The PEP-II storage ring}

\subsubsection{Introduction}

The PEP-II storage ring [35] is an $e^{+} e^{-}$collider designed to operate at the $\Upsilon(4 \mathrm{~S})$ resonance, with a corresponding $\mathrm{CM}$ energy of $10.58 \mathrm{GeV}$. The $\Upsilon(4 \mathrm{~S})$ resonance is a unique source of neutral $B_{d}^{0}$ mesons, lying just above production threshold and below the necessary CM energy to produce $B_{s}^{0}$ mesons. The ratio of $\Upsilon(4 \mathrm{~S})$ production to $q \bar{q}$ production is approximately $1: 3$, and the $\Upsilon(4 \mathrm{~S})$ decays into neutral $B$ mesons approximately $50 \%$ of the time. The requirements that $B$ meson final states of small branching ratio be reconstructed and that the separation of the two $B$ meson decays be measured are reflected in the principal differences of PEP-II with respect to existing colliders. These are the high design luminosity of $3 \times 10^{33} \mathrm{~cm}^{-2} \mathrm{~s}^{-1}$ and the asymmetry, colliding 


\begin{tabular}{|c|c|c|}
\hline & Low energy ring & High energy ring \\
\hline Energy $(\mathrm{GeV})$ & 3.1 & 9.0 \\
Circumference $(\mathrm{m})$ & 2199.32 & 2199.32 \\
Emittance at IP $\varepsilon_{x} / \varepsilon_{y}(\mathrm{~nm} \mathrm{rad})$ & $64.3 / 2.6$ & $48.2 / 1.9$ \\
Beta function at IP $\beta_{x}^{*} / \beta_{y}^{*}(\mathrm{~cm})$ & $37.5 / 1.5$ & $50.0 / 2.0$ \\
Beam-beam tune shift $\xi_{0, x} / \xi_{0, y}$ & $0.03 / 0.03$ & $0.03 / 0.03$ \\
RF frequency (MHz) & 476 & 476 \\
RF voltage (MV) & 5.9 & 18.5 \\
Bunch length (cm) & 1.0 & 1.0 \\
Number of bunches & 1658 & 1658 \\
Bunch separation (m) & 1.26 & 1.26 \\
Total current (A) & 2.14 & 0.99 \\
\hline
\end{tabular}

Table 2-1. $\quad$ Parameters of the PEP-II asymmetric storage ring.

9.0 $\mathrm{GeV}$ electrons with $3.1 \mathrm{GeV}$ positrons. These differences impose quite severe demands on the machine and have an impact on detector design and performance. In the first two sections of this chapter key components of the accelerator and storage ring will be described. A more detailed description of the interaction region will then be given, and the section will conclude with a discussion of the machine's impact on detector performance in the form of machine backgrounds.

\subsubsection{The Main Storage Ring}

Following the original suggestion that an asymmetric collider could provide adequate vertex resolution to study $C P$ violation at the $\Upsilon(4 \mathrm{~S})[36]$, further studies [37, 38, 39] indicated that an asymmetry in the region of $1: 3$ would be optimal. The cross section at the $\Upsilon(4 \mathrm{~S})$ is approximately $1.1 \mathrm{nb}$ on a background of $2.5 \mathrm{nb}$, giving a luminosity requirement of $3 \times 10^{33} \mathrm{~cm}^{-2} \mathrm{~s}^{-1}$ in order to produce the required $3 \times 10^{7} B$ mesons per year. These are the essential design requirements of the PEP-II storage ring. The parameters chosen to achieve these requirements are shown in table $2-1$.

The selected energies of $3.1 \mathrm{GeV}$ (positrons) and $9.0 \mathrm{GeV}$ (electrons) give a $\beta \gamma$ to the $\Upsilon(4 \mathrm{~S})$ of 0.56 , which in turn give a mean separation of the decay points of the two $B^{0} \mathrm{~s}$ of $250 \mu \mathrm{m}$. This is well within the resolution of current vertex detectors. 
The luminosity of the machine is given by [40]:

$$
\mathcal{L}=2.17 \times 10^{34} \xi(1+r)\left(\frac{I E}{\beta_{y}^{*}}\right) \mathrm{cm}^{-2} \mathrm{~s}^{-1}
$$

where $I$ is the beam current in amperes, $E$ is the beam energy in $\mathrm{GeV}, \beta_{y}^{*}$ is the vertical betatron function at the interaction point (IP), $r$ is the ratio of beam height to beam width at the IP (the beam aspect ratio) defined in terms of the betatron functions and emittances $\left(\varepsilon_{x, y}\right)$ thus:

$$
r=\left(\frac{\varepsilon_{y} \beta_{y}^{*}}{\varepsilon_{x} \beta_{x}^{*}}\right)^{1 / 2}
$$

and $\xi$ is the beam-beam tune shift of the machine. From this equation, it can be seen that high beam-beam tune number, low betatron function, high current and rounded beams are desirable. However, machine constraints and detector considerations must also be taken into account. The beam-beam tune shift of 0.03 is chosen as a conservative number lying well within the range of existing colliders (0.028-0.055 [9]). The beam aspect ratio has a maximum at one. However this case of round beams has practical difficulties, notably the need for strong focusing quadrupoles near the IP which impacts on detector design, principally because of the accompanying increase in synchrotron radiation and decrease in acceptance. An aspect ratio of 0.04 has been chosen for PEP-II. To achieve high luminosity, then, a small vertical betatron function and high currents are employed. The currents are of particular note, being an order of magnitude larger than existing machines. This gives large machine backgrounds, which will be discussed in section 2.2.5.

The RF parameters are also closely linked. The frequency is chosen to be $1 / 6$ th that of the operating frequency of the SLAC linac $(2.856 \mathrm{GHz})$ such that the phase of the two systems may be locked together (see section 2.2.3) whilst remaining close to the operating frequency of commercially available RF systems (typically $500 \mathrm{MHz}$ ). The bunch length, $\sigma_{l}$, is constrained physically to be less than the vertical betatron function at the IP, and luminosity lifetime considerations give the choice of $1 \mathrm{~cm}$. The operating voltage is then fixed by the relation

$$
\sigma_{l} \propto \frac{1}{\sqrt{V_{R F} f_{R F}}} .
$$

The magnetic bending radius for the high (low) energy ring is $165 \mathrm{~m}(13.75 \mathrm{~m})$ respectively. The relation [41]

$$
P_{S R}=\frac{88.5 E^{4} I}{\rho} \mathrm{k} W
$$

where $P_{S R}$ is the radiated synchrotron power and $\rho$ is the magnetic bending radius, allows the power consumption of the two rings to be calculated as 3.58MW (HER) and 1.24MW (LER). In fact, more synchrotron radiation is lost in the interaction region (this fraction is substantial for the LER), and the total power required is 3.69 (2.57) MW for the HER (LER). Given additional losses in the transmission of klystron power to the beam, the final number of klystrons is chosen as 10 (5) delivering 11.0(5.5)MW for the HER (LER).

Synchrotron radiation is the dominant effect in the consideration of residual gas pressure in the beam pipe. The thermal load in the HER is of the order of $100 \mathrm{~W} / \mathrm{cm}$, which gives a substantial 
desorption rate into the vacuum chamber. This desorption rate is a function of the energy of the beam, the beam current and a quantity known as the photodesorption coefficient, $\eta$. This quantity is not well defined, being a function of the photon angle of incidence, radiation damage and manufacturing technique as well as the incident energy and the material itself. However, experimental results show that this quantity is at least an order of magnitude less in copper and stainless steel than in the more easy to manufacture aluminium. Copper vacuum chambers were chosen for the arcs of the machine, whilst stainless steel ones (having a slightly higher $\eta$ but being cheaper) were chosen for the straight sections. The overall pressure requirements, derived from background considerations near the IP and beam lifetime considerations elsewhere, are that the $N_{2}$ equivalent pressures be:

- $\leq 10 \mathrm{nTorr}$ in the arcs

- $\sim 3$ nTorr in the straight sections

- $\sim 1$ nTorr in the straight section of the IP.

- $\sim 0.2 \mathrm{nTorr}$ within $\pm 20 \mathrm{~cm}$ of the IP.

The choice of vacuum chamber material brings these numbers well within the range of existing pump technology.

The magnet systems (excepting those in the interaction region which will be discussed in section 2.2.4) either use directly the existing magnets of PEP, or closely follow their design. The PEP ring was designed to handle beam energies of up to $18 \mathrm{GeV}$ and is thus well suited to this purpose.

The very large number of bunches and large beam currents pose particular problems for PEP-II. Aside from the problems of synchrotron radiation and beam backgrounds, the beams themselves can cause excitations in the RF or other components that they pass through, leading to beam instabilities. To address this problem, PEP-II is employing a feedback system which measures the position of a bunch relative to its nominal position, and then provides up to $2.8 \mathrm{kV} / \mathrm{turn}$ of damping voltage to the same bunch to rectify any bunch instability. This requires a very high bandwidth digital filter which compares the bunch profile to the ideal (a sinusoid at $238 \mathrm{MHz}$ ) and applies the appropriate correction.

\subsubsection{The Injection System}

The injection system for PEP-II has as its principle component the SLAC linac. This operates in a two cycle mode. The first cycle accelerates an electron bunch which is injected into PEPII, and another electron bunch is stored in one of the linac damping rings. On the next cycle, the positron bunch is accelerated, followed by the stored electron bunch. The positron bunch is separated off at the appropriate energy, while the following electron bunch is accelerated to high energy (about $30 \mathrm{GeV}$ ) to provide the next positron bunch. The accelerated bunches do not require 
the full length of the linac to acquire the appropriate energy and are transmitted to the storage ring via non-accelerating transmission lines. The pulse rate is $60 \mathrm{~Hz}$.

Beam lifetime considerations mean that a top up ( $80 \%$ to $100 \%)$ every hour will be necessary during factory running. Luminosity requirements dictate that this occur inside a 3 minute time window. This requires that each pulse in the above description carry $0.3 \times 10^{10} e^{ \pm}$, which is to be compared with the $3 \times 10^{10} e^{ \pm}$regularly delivered by the SLAC linac to the SLC collider.

The injection scheme itself is a vertical one, so chosen to reduce the number of parasitic beam crossings that would occur at the IP due to synchrotron oscillations of off momenta particles in the horizontal plane. The location for injection is chosen to be at large $\beta$, to give the maximum efficiency for acceptance of the injected particles into the circulating beams. The momentum resolution of the SLAC linac injector gives a $\delta p / p \simeq 0.7 \%$ (FWHM) which matches well onto the RF acceptance of PEP-II.

\subsubsection{The Interaction Region}

The asymmetry and high luminosity of PEP-II pose particular difficulties in the interaction region. The asymmetry means that the beams must be kept apart even relatively close to the IP. The components in the interaction region are shown in figure 2-1. The first magnet encountered after the IP is a dipole (B1) which gives a horizontal separation to the beams. Of the magnets in the IP region, these have the most significant impact on the acceptance of the $B A B A R$ detector, which is consequently constrained to lie in the region $\left|\cos \theta_{l a b}\right|<0.955$. The next two magnets are quadrupoles (Q1, Q2), which are centred on the LER. The first magnet focuses in the vertical direction the LER travelling towards the IP (some focusing of the HER is achieved), but by the next quadrupole, situated $3 \mathrm{~m}$ from the IP, the HER has sufficient separation from the LER to pass through a constant field region of the magnet, which is focusing in the horizontal direction for the LER. The main focusing magnets for the HER are situated between $4 \mathrm{~m}$ and $7 \mathrm{~m}$ from the IP, with a small dipole at $3.7 \mathrm{~m}$ to ensure that the synchrotron radiation generated by the focusing quadrupoles does not shine on the interaction point. These magnets have no effect on the LER which continues to undergo horizontal separation, reaching a maximum distance of $2.8 \mathrm{~m}$ from the HER at $40 \mathrm{~m}$ from the IP, where more dipoles return it to the same horizontal position as the HER. Between $22 \mathrm{~m}$ and $60 \mathrm{~m}$, the LER is also separated vertically from the HER by $1 \mathrm{~m}$, which allows the two to be at the same radius for the rest of the ring.

The r.m.s. beam sizes at the IP are $155 \mu \mathrm{m}$ in the horizontal direction and $6.2 \mu \mathrm{m}$ in the vertical direction. 


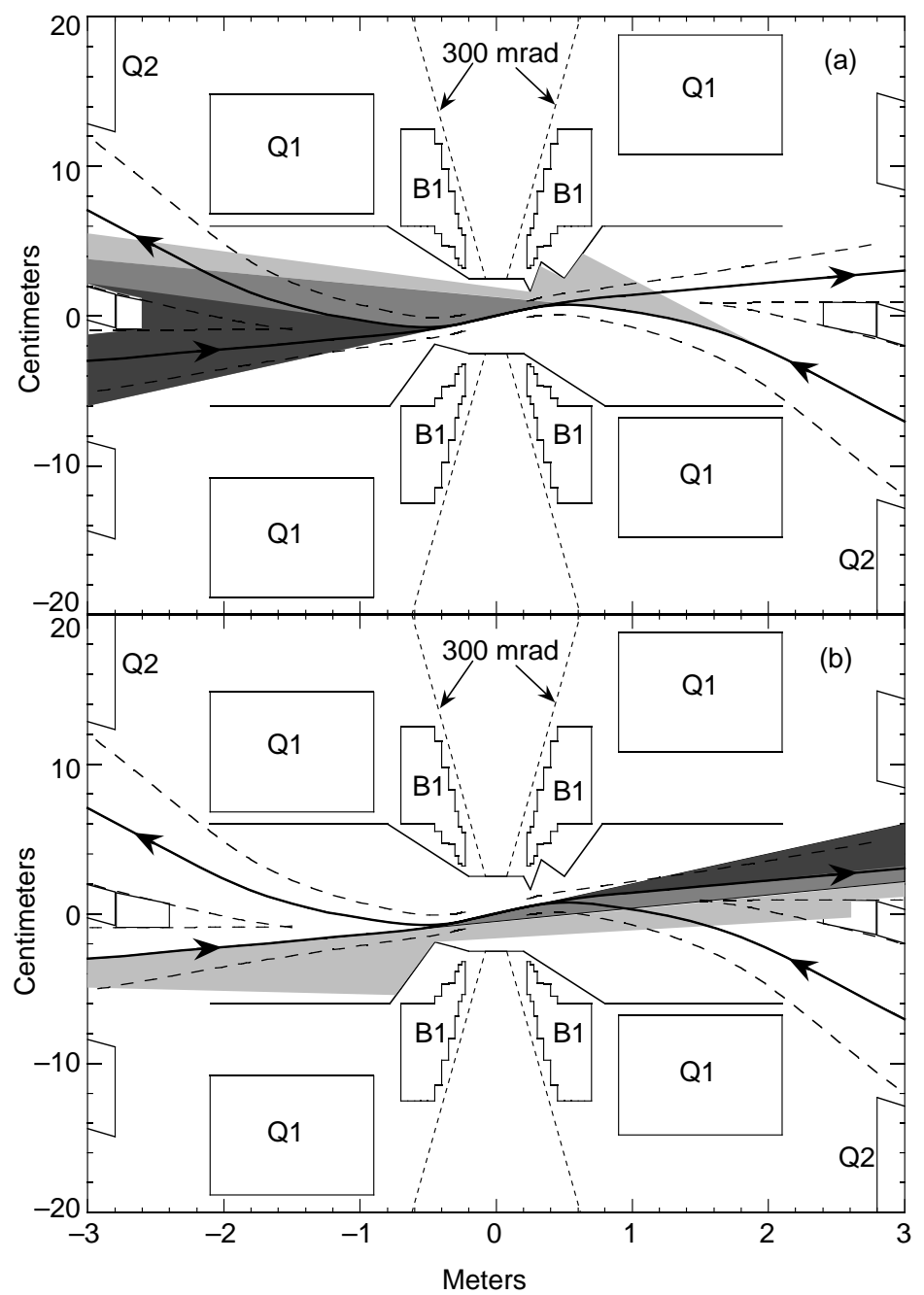

Figure 2-1. Synchrotron radiation deposits from the low energy beam (top) and high energy beam (bottom). The Q1 quadrupole, being off axis w.r.t the low energy beam, and the B1 separation dipoles are the principle sources of synchrotron radiation at the IP. The darker shading indicates regions of higher photon density. 


\subsubsection{Machine backgrounds}

The machine backgrounds in PEP-II are problematic because of the high beam currents employed. These are typically an order of magnitude larger than previous machines, but in order not to impact on detector performance, the backgrounds should be no larger than those typically observed in the lower luminosity storage rings such as CESR[42]. There are three principal sources of machine background: synchrotron radiation, lost beam particles due to bremsstrahlung with residual gas molecules and lost beam particles due to Coulomb scattering off residual gas molecules. The detector performance is impacted either by occupancy levels being too high or radiation damage to detector components. The acceptable background levels have been calculated [35] and are given for the two tracking chambers (see sections 2.3.1 and 2.3.2) as an occupancy rate of $10 \%$ for each, and a radiation dose of $20 \mathrm{krad} / \mathrm{year}$ for the silicon vertex tracker, and $0.5 \mathrm{C} /$ year per wire in the drift chamber.

\subsubsection{Synchrotron radiation background}

There are two types of synchrotron radiation: synchrotron radiation caused by the beam passing through a dipole magnet or an offset quadrupole magnet, in which all particles radiate in a fan shape projecting forwards from the beam-referred to as fan radiation-and that caused by off axis particles passing through an on axis quadrupole or sextupole (generally at least $3 \sigma$ from the nominal beam position), referred to as quadrupole radiation. Studies have shown [35] that the latter type contributes less than $1 \%$ of the synchrotron radiation rate at PEP-II, and so here we will only consider the former. Further, the dominant contribution to the synchrotron radiation rates near the IP are the dipole bending magnets used to split the beams horizontally, described in section 2.2.4, which give $6.18 \mathrm{~kW}$ and $24.0 \mathrm{~kW}$ of deposited energy within $50 \mathrm{~cm}$ of the IP, and the first focusing quadrupole which, whilst the high energy ring passes through a field free region of this magnet, deposits $2.88 \mathrm{~kW}$ in the same region due to the low energy ring. A copper mask is placed on the exposed region of the beampipe near to the horizontal separation dipoles. This mask reduces the occupancy and radiation rates to about 1/80th of their acceptable levels. The source of the synchrotron radiation fans and the masks to shield the detector from them is shown in figure $2-1$.

\subsubsection{Lost beam particle backgrounds}

The lost beam particle backgrounds from bremsstrahlung and Coulomb scattering are expected to be a significant source of detector occupancy and radiation damage. The beam particles striking residual gas and undergoing these reactions may fall outside the momentum acceptance range of the storage ring, and thus strike the beam pipe, causing an electromagnetic shower. The rate of this occurrence has been simulated[43] using Decay TURTLE [44], which models the passage of the beam through the accelerator elements before and after undergoing such an interaction. The 
output from this simulation, which gives the energy and position along the beampipe of the lost particles, is then fed into a full GEANT[45] simulation of the $B A B A R$ beampipe and detector.

\begin{tabular}{|c|c|c|c|c|c|c|}
\hline & \multicolumn{2}{|c|}{ Silicon Vertex } & \multicolumn{2}{c|}{ Drift Chamber } & \multirow{2}{*}{ DIRC } & \multirow{2}{*}{ Calorimeter } \\
\cline { 2 - 5 } & Average & Inner layer & Average & Inner layer & & Photons \\
\hline $\begin{array}{c}\text { Occupancy } \\
\text { Limit }\end{array}$ & $1.3 \%$ & $3.0 \%$ & $0.05 \%$ & $0.5 \%$ & $0.015 \%$ & $1.1 / \mu \mathrm{s}$ \\
$10 \%$ & $10 \%$ & $2 \%$ & $20 / \mu \mathrm{s}$ \\
\hline Rad. dose (yr) & $33 \mathrm{krad}$ & $82 \mathrm{krad}$ & $4 \times 10^{-4} \mathrm{C} / \mathrm{cm}$ & $4.5 \times 10^{-3} \mathrm{C} / \mathrm{cm}$ & & $1.5 \mathrm{krad}$ \\
Limit & $1500 \mathrm{krad}$ & $1500 \mathrm{krad}$ & $0.1 \mathrm{C} / \mathrm{cm}$ & $0.1 \mathrm{C} / \mathrm{cm}$ & & $100 \mathrm{krad}$ \\
\hline
\end{tabular}

Table 2-2. Occupancy rates and radiation doses from lost beam particle backgrounds in the four innermost sections of the BABAR experiment.

The lost particle rate from bremsstrahlung interactions are approximately ten times those from Coulomb interactions. The particles which strike the beam pipe near the IP can originate from several tens of metres away from the IP, and this makes it imperative that the pressure be as low as possible in the IR straight. The synchrotron radiation in the arcs makes a reduction of pressure in these regions more difficult, but the bending dipoles ensure that off momentum particles are more rapidly lost in these regions, and thus has negligible impact on detector performance.

The background rates observed by the tracking systems and the calorimeter are shown in table 2-2, with an indication of the safety margin available for each device. The instrumented flux return (see section 2.3.5) is assumed to be adequately shielded from such background by the other devices.

\subsection{The $B_{A} B_{A R}$ experiment}

The $B A B A R$ experiment is a state of the art, general purpose collider detector, optimised for determination of $C P$ violating $B$ meson decays. It has 5 principle components: a vertex detector, a tracking chamber, a particle identification device (the DIRC), an electromagnetic calorimeter and an instrumented flux return used for $\mu$ and $K_{L}^{0}$ identification. The calorimeter, DIRC and tracking devices are situated inside a 1.5T superconducting solenoid. This section will describe each of the 5 subdetectors in detail. Further information on the calorimeter, in particular on its readout and projected performance, can be found in subsequent chapters. 


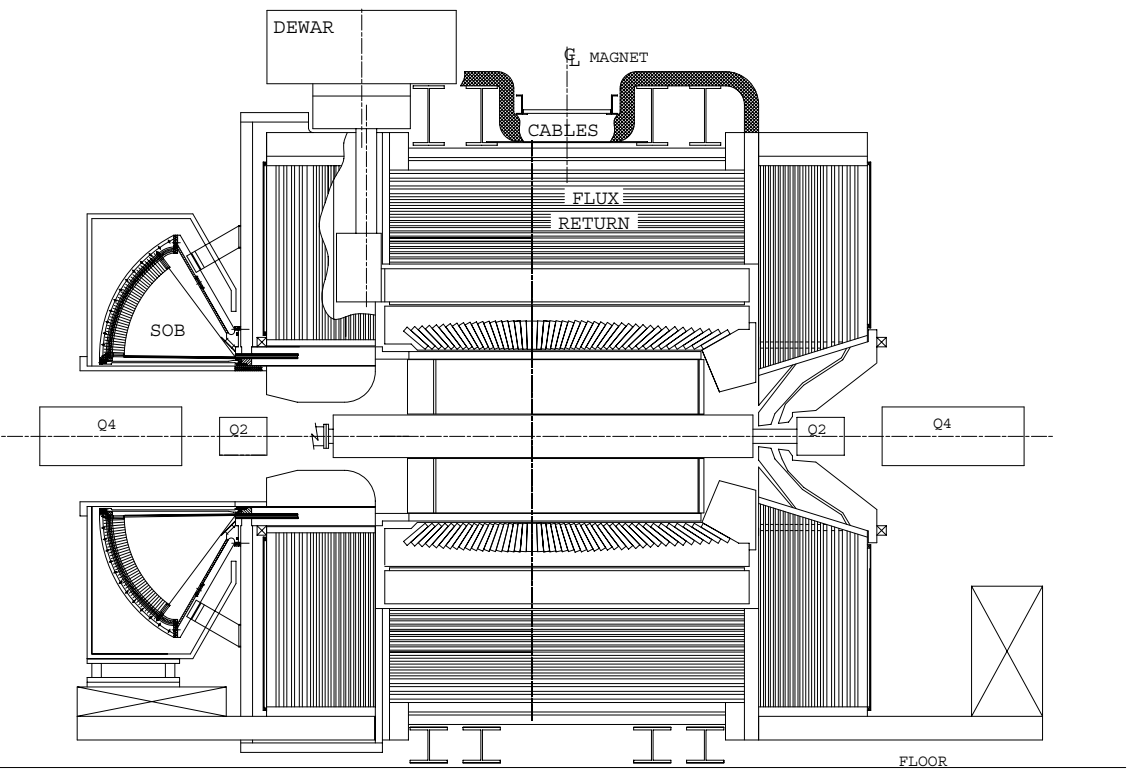

Figure 2-2. The BABAR detector. The $9.0 \mathrm{GeV}$ electron beam travels left to right, the $3.1 \mathrm{GeV}$ positron beam right to left. The interaction point is marked by the crosshairs.

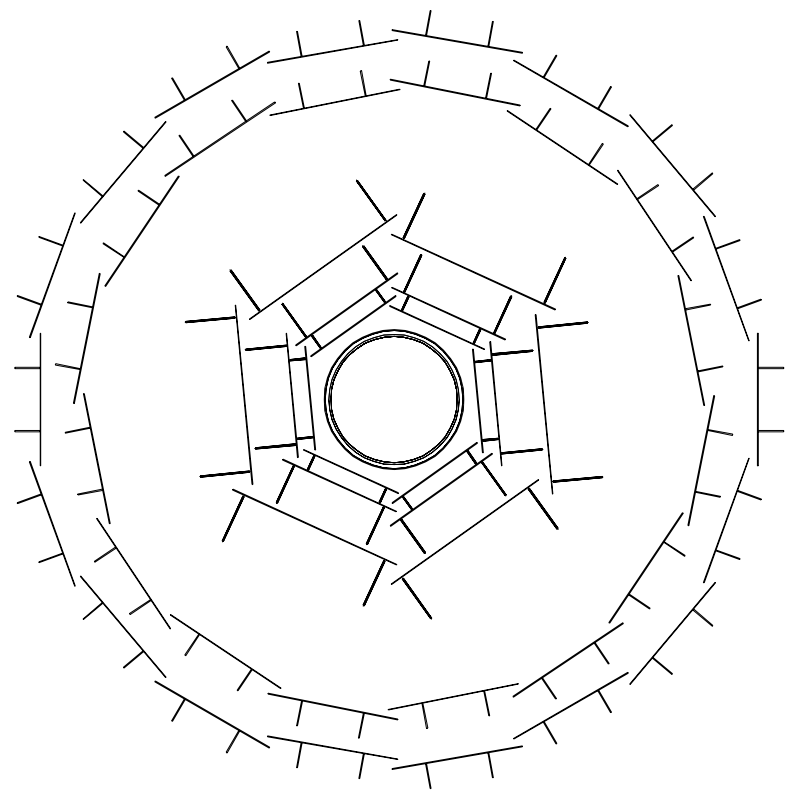

Figure 2-3. The BABAR silicon vertex tracker. Cross sectional view in the $r / \phi$ plane. The beam pipe is shown in the centre. 


\subsubsection{The Silicon Vertex Tracker}

\subsubsection{Overview}

The precise measurement of the decay vertices of the two $B$ mesons, and in particular their separation, is of crucial importance to the measurement of $C P$ violation. Studies have shown [46] that the resolution necessary on the separation of the two vertices is of order $80 \mu \mathrm{m}$ on a quantity whose mean is $250 \mu \mathrm{m}$. This has been shown to be easily achievable using existing technology [47]. The BABAR silicon vertex detector has been designed to provide the best information possible on the vertex separation whilst minimising multiple scattering of tracks which enter the drift chamber. It is a five layer double sided device whose design allows for the optimal solid angle coverage within the constraints of the interaction region magnets. The expected resolution is $\sim 50 \mu \mathrm{m}$ at $90^{\circ}$ at $1 \mathrm{GeV} / \mathrm{c}$. The detector will also provide tracking information in addition to the drift chamber, and will be the only tracking detector for those tracks with $p<100 \mathrm{MeV} / \mathrm{c}$.

\subsubsection{Detector Layout}

The physical layout of the silicon vertex tracker is shown in figures 2-3 and 2-4. The SVT consists of 5 approximately concentric layers: 3 barrel type layers close to the IP, and 2 arch type layers at a slightly larger radial distance. The inner two layers are primarily designed for impact parameter determination, the outer two layers for precision alignment with tracks in the drift chamber, and the middle layer to assist in pattern recognition. The arched nature of the outer layers allows for better solid angle coverage and decreases the probability of a large impact angle for tracks in the forward and backward regions with consequent reduction in multiple scattering. The solid angle coverage is restricted by the beam separating dipole magnets and the space for mechanical support and electronics to $350 \mathrm{mr}$ in the forward direction to 2.621 radians in the backward direction.

\begin{tabular}{|c|c|c|c|c|c|c|c|}
\hline Layer & 1 & 2 & 3 & $4 \mathrm{a}$ & $4 \mathrm{~b}$ & $5 \mathrm{a}$ & $5 \mathrm{~b}$ \\
\hline Radius (mm) & 32 & 40 & 54 & 124 & 127 & 140 & 144 \\
Modules/layer & 6 & 6 & 6 & 8 & 8 & 9 & 9 \\
Wafers/module & 4 & 4 & 6 & 7 & 7 & 8 & 8 \\
\hline Intrinsic resolution $(\mu \mathrm{m})$ & & & & & \\
$z$ & 10 & 10 & 10 & $10-12$ & $10-12$ \\
$\phi$ & 12 & 12 & 12 & 25 & 25 \\
\hline
\end{tabular}

Table 2-3. Description of the layers of the silicon vertex tracker. 


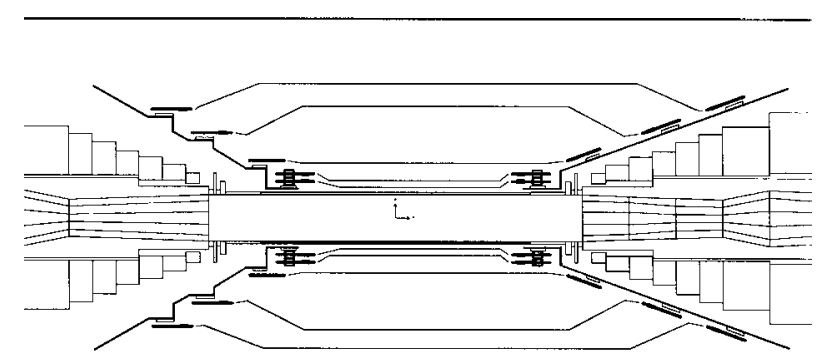

$10 \mathrm{~cm}$

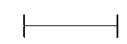

Figure 2-4. The BABAR silicon vertex tracker. Cross sectional view in the $z$ plane. The interaction point is marked, indicating the asymmetry of PEP-II. The structures to the right and left are the beam separating fixed dipole magnets.

The layers consist of double sided orthogonal readout strips measuring the $z$ and $\phi$ co-ordinates on the inner and outer sides respectively. The inner 3 layers are divided into 6 detector modules in $\phi$, which are displaced slightly in $\phi$ to allow for overlap at the detector edges. Layers 4 and 5 are divided into 16 and 18 modules respectively, which are again displaced to allow for detector overlap, but radially allowing for a 9 fold $\phi$ symmetry. Each layer is divided into forward and backward half modules which are electrically isolated and then further divided into the individual silicon wafers. The properties of each layer are listed in table 2-3 and those of each wafer are listed in table 2-4.

\begin{tabular}{|c|c|c|c|c|c|c|}
\hline Wafer Type & I & II & III & IV & V & VI \\
\hline $\begin{array}{c}\text { Dimensions (mm) } \\
z\end{array}$ & 42.40 & 45.40 & 43.90 & 67.55 & 53.90 & 67.97 \\
$\phi$ & 41.30 & 49.42 & 71.47 & 52.50 & 52.50 & $52.80 \rightarrow 43.30$ \\
\hline $\begin{array}{c}\text { Readout pitch ( } \mu \mathrm{m}) \\
z\end{array}$ & 100 & 100 & 100 & 210 & 210 & 210 \\
$\phi$ & 50 & 55 & 55 & 100 & 100 & $100 \rightarrow 82$ \\
\hline Number of strips & & & & & & \\
$z$ & 411 & 441 & 426 & 316 & 251 & 318 \\
$\phi$ & 799 & 874 & 1275 & 512 & 512 & 512 \\
\hline
\end{tabular}

Table 2-4. Description of the wafers constituting the silicon vertex tracker. 


\subsubsection{Electronics and readout}

The signal from each strip is amplified and passed through a CR-RC ${ }^{2}$ shaping circuit. The time for which the resulting signal is over a given threshold (to be determined by background conditions during running) is then digitised. This 'time over threshold' varies approximately logarithmically with deposited charge, giving a larger dynamic range for a given bandwidth than a readout of the full signal. The digitisation is performed as part of the front end electronics. The digitised signal is fed into a circular buffer which is read out when a level 1 trigger accept signal is received.

The strips themselves are biased to $+20 \mathrm{~V}$, with a maximum allowable voltage of $60 \mathrm{~V}$. The interstrip resistance is $100 \mathrm{M} \Omega$, and a leakage current of $1 \mu \mathrm{A}$ per strip per $\mathrm{cm}^{-2}$ can be tolerated, providing that the total leakage current for each layer does not exceed $15 \mu \mathrm{A}$.

\subsubsection{The Drift Chamber}

The drift chamber is the central tracking detector in $B A B A R$. Its primary purpose is to provide accurate and efficient measurement of charged tracks necessary to provide exclusive reconstruction of $B$ meson decays. Good spatial resolution must be achieved not only to provide the momentum measurement, but also to ensure good matching of tracks to those measured in the silicon vertex tracker, and to provide information on charged tracks to the DIRC and electromagnetic calorimeter. The other functions of the drift chamber are to provide input to the trigger system, and to provide $\mathrm{dE} / \mathrm{dx}$ information on lower momentum particles $(\sim 700 \mathrm{MeV} / \mathrm{c}$ or below) where BABAR's primary particle identification device, the DIRC, is less sensitive. The target spatial and momentum resolution are $140 \mu \mathrm{m}$ and $0.3 \% \times p_{\mathrm{t}}$ for tracks with $p_{\mathrm{t}}$ above $1 \mathrm{GeV} / \mathrm{c}$, respectively. The drift chamber must present the minimum possible material to the DIRC and calorimeter.

\subsubsection{Mechanical design}

A cross sectional view of the drift chamber is shown in figure 2-5. The chamber consists of 7104 hexagonal cells, of approximately $1.7 \times 1.2 \mathrm{~cm}$, arranged into 10 superlayers of 4 layers each. The arrangement of axial (A) and stereo $(\mathrm{U}, \mathrm{V})$ layers is shown in figure 2-6 and table 2-5 gives the numbers of cells per layer. The stereo angle varies between $40 \mathrm{mr}$ for the inner layer to $70 \mathrm{mr}$ for the outer layer. The sense wires are $20 \mu \mathrm{m}$ gold plated tungsten-rhenium and carry $2020 \mathrm{~V}$, while the field shaping wires are $120 \mu \mathrm{m}$ and $80 \mu \mathrm{m}$ gold plated aluminium carrying 350V. The inner wall of the chamber is a $1 \mathrm{~mm}$ beryllium cylinder of radius $23.6 \mathrm{~cm}$, and the outer wall consists of two layers of carbon fibre on a Nomex core. The gas in the chamber is a 4:1 mix of $\mathrm{He}: \mathrm{C}_{4} \mathrm{H}_{10}$, chosen to give the best possible $\mathrm{dE} / \mathrm{dx}$ information and reasonably low drift times whilst minimising the material in front of the outer detectors. The total amount of material at $90^{\circ}$ is $2.08 \% X_{0}$. 


\begin{tabular}{|c|c|c|}
\hline Superlayer (4 layers) & Stereo (U,V)/ axial(A) & Number of cells per layer \\
\hline 1 & A & 96 \\
2 & U & 112 \\
3 & V & 128 \\
4 & A & 144 \\
5 & U & 176 \\
6 & V & 192 \\
7 & A & 208 \\
8 & U & 224 \\
9 & V & 240 \\
10 & A & 256 \\
\hline
\end{tabular}

Table 2-5. Description of the drift chamber superlayers.

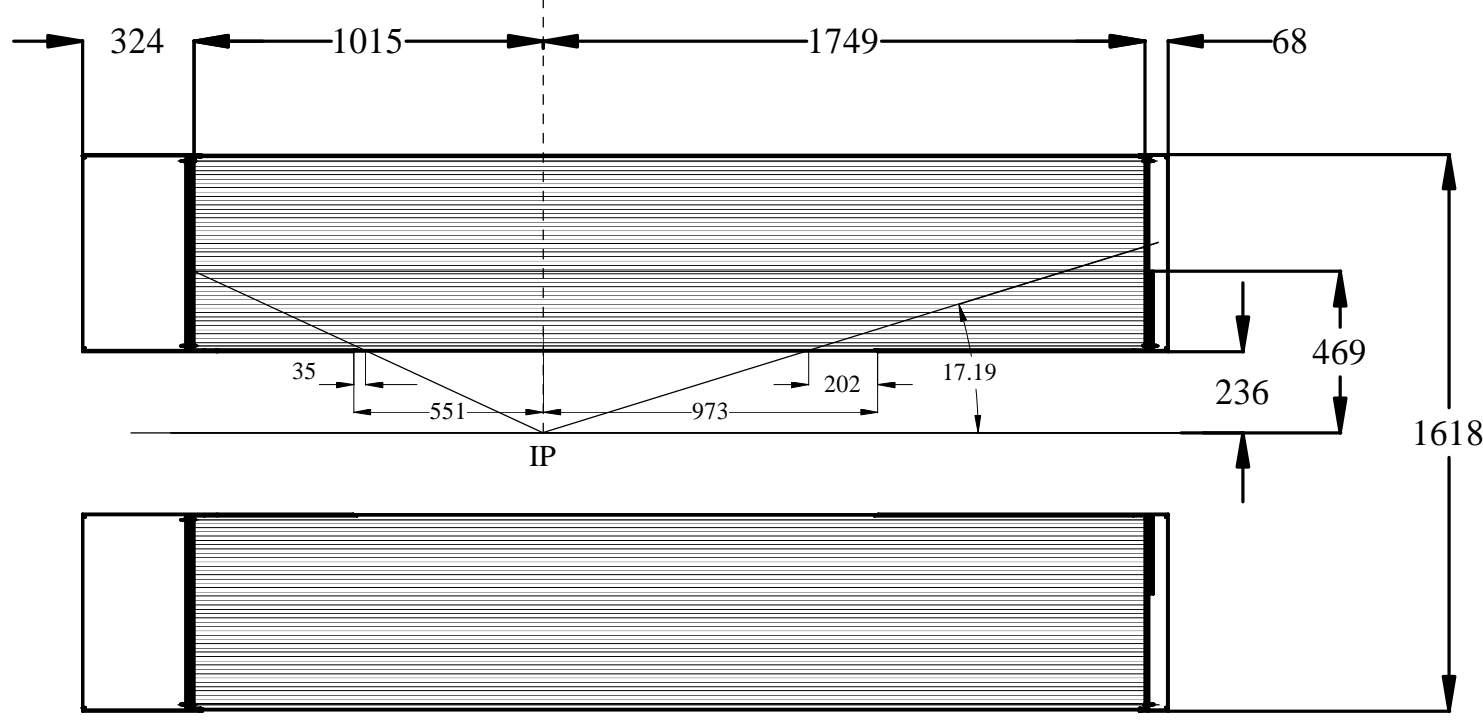

Figure 2-5. The BABAR drift chamber ( $z$ projection). The chamber is offset in $z$ by $367 \mathrm{~mm}$ to account for the asymmetry. The dimensions shown are in $\mathrm{mm}$. 


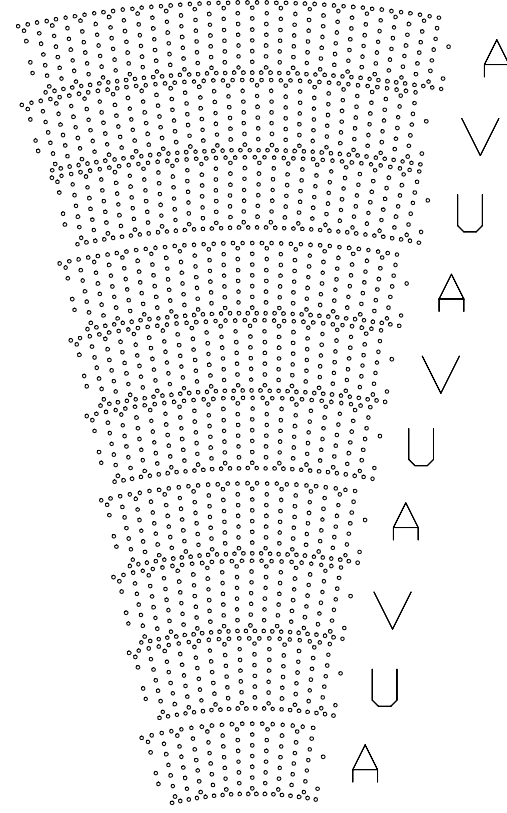

Figure 2-6. Section of the cell layout for the drift chamber. Axial layers are labeled A, stereo layers $U$ and $V$.

The asymmetry of the calorimeter (see section 2.3.4) requires that the drift chamber material be minimised in the forward direction whilst allowing some scope for an increased amount in the backward direction. To this end, the forward endplate is only half the thickness of the rear one at $12 \mathrm{~mm}$, within the calorimeter acceptance, and the readout electronics for the drift chamber are mounted on the rear endplate. The endplates are constructed from aluminium.

\subsubsection{Electronics}

The requirements on the drift chamber electronics are that the total charge and the drift time are read out to both the data acquisition system and trigger with a degradation on the performance of the chamber of no more than $10 \%$. The total charge is necessary for $\mathrm{dE} / \mathrm{dx}$ measurements, whilst the drift time measurement provides positional information.

The amplification, digitisation and data formatting for the drift chamber are all done on the rear endplate behind the high voltage assembly. Each sense wire passes through a high voltage isolation capacitor, and 4 such channels are then passed to an amplifier. The signal then proceeds through two paths: one to a shaping circuit which is digitised by a 6 bit flash ADC operating at 14.875 $\mathrm{MHz}$, the other to a discriminating circuit which is digitised by a TDC providing $1 \mathrm{~ns}$ precision. The digitisation chip takes the output from 8 amplification circuits. The output is then placed in a 
buffer of $12.9 \mu$ s latency which is emptied in to an event buffer when given an accept signal from the level 1 trigger. This double buffering is necessary to prevent dead time in the chamber. The event buffers are read out by 2 bit $30 \mathrm{MHz}$ lines to data IO modules which pass the data to the $B A B A R$ DAQ system.

A full description of the drift chamber electronics can be found in [48].

\subsubsection{The DIRC}

Particle identification is a crucial part of the $B A B A R$ experiment. The need to tag the flavour of the $B$ meson which does not decay into $C P$ eigenstate gives rise to the need to distinguish between charged $K$ and $\pi$ at momenta up to about $2 \mathrm{GeV} / \mathrm{c}$. In addition, there are several $C P$ channels where $K / \pi$ separation is essential. A notable example is the decay $B \rightarrow \pi^{ \pm} \pi^{\mp}$, which gives information on the angle $\alpha$. In order to distinguish this channel from $B \rightarrow K^{ \pm} \pi^{\mp}, K / \pi$ discrimination is necessary up to $4 \mathrm{GeV} / \mathrm{c}$. The particle ID system must be able to function in conditions of high machine background and have as little impact as possible on the precision of the electromagnetic calorimeter situated behind it.

The particle ID system chosen in $B A B A R$ is one based on the concept of totally internally reflected Čerenkov radiation. The principle is illustrated in figure 2-7. TheČerenkov radiation is emitted at a characteristic angle $\theta_{c}$ given by $\theta_{c}=(\beta n)^{-1}$ where $n$ is the refractive index of the medium (see for example [49]). BABAR has chosen a quartz radiator for its high refractive index ( $n=1.474$ ) which preserves more of the internally reflected radiation, its long radiation length $(11.7 \mathrm{~cm})$, its long absorption length for the characteristic UV light, and its ability to be finished to a high quality optical surface.

The difference between the Čerenkov angle at $4.0 \mathrm{GeV} / \mathrm{c}$ for pions and kaons is about $6.5 \mathrm{mr}$. This gives stringent requirements on performance, specifically that the Čerenkov angle be resolved to better than $2 \mathrm{mr}$.

\subsubsection{Mechanical construction}

The DIRC (Detector of Internally Reflected Čerenkov light) is constructed in the form of a twelve sided regular polygon. Each side consists of 48 rectangular bars separated by $75 \mu \mathrm{m}$. The dimensions of the bars are $1.7 \times 3.5 \times 122.5 \mathrm{~cm}$, and 4 such bars are glued together to make a total length of $4.9 \mathrm{~m}$. The material in front of the calorimeter due to the quartz is therefore $0.15 \mathrm{X}_{o}$ at $90^{\circ}$. The bars are held in an aluminium frame which is held at the rear of the detector in a cantilever arrangement. The frame presents an additional $0.05 \mathrm{X}_{\circ}$ at $90^{\circ}$. The azimuthal angle coverage is $94 \%$, and that in the centre of mass polar angle is $87 \%$.

To avoid adding to the material in the forward region where there is a concentration of centre of mass solid angle, the DIRC is instrumented at the back. Any Čerenkov light emitted in the 


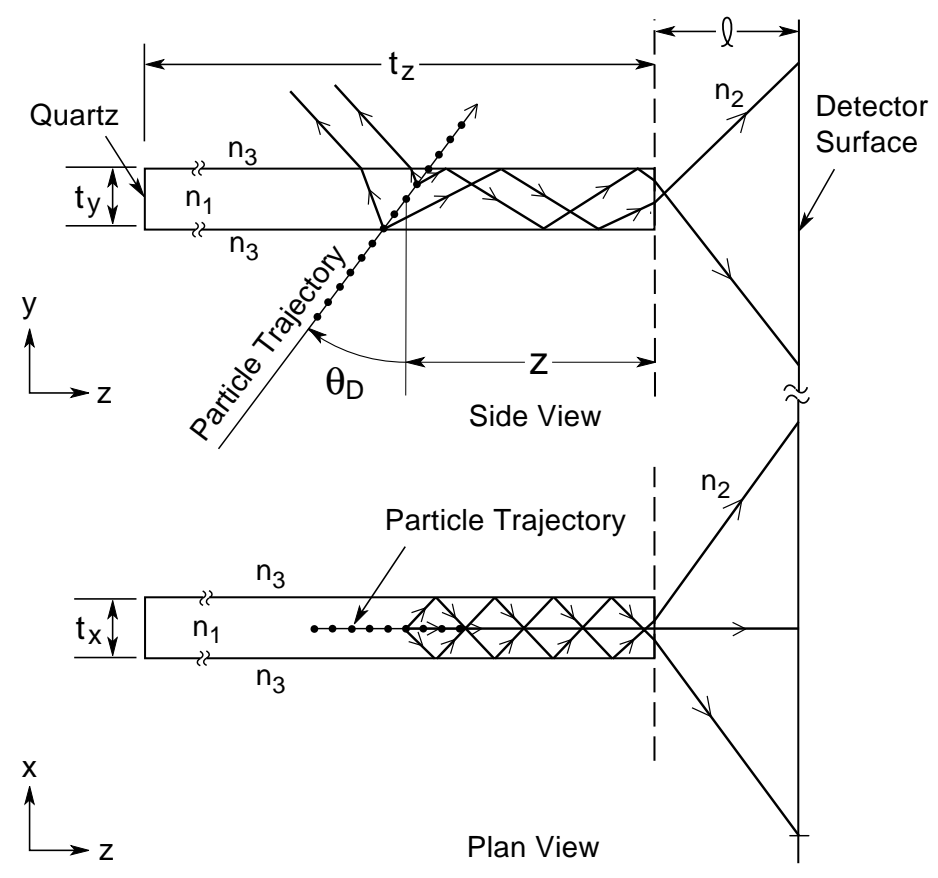

Figure 2-7. The BABAR DIRC concept. Čerenkov radiation is internally reflected in the quartz bar, and emitted at the end of the bar preserving the Čerenkov angle.

forward direction is reflected by means of a quartz wedge situated at the end of the bar, designed to return the light towards the rear while preserving the original Čerenkov angle. This gives a two fold ambiguity in the imaging of the Čerenkov cone, but this can be resolved by using timing information and the position of the charged track given by the drift chamber.

\subsubsection{Readout}

The Čerenkov light emitted at the back of the DIRC is allowed to expand in a region of purified water (so chosen to have a refractive index close to that of quartz) and is detected by an array of $11,0002.82 \mathrm{~cm}$ diameter photomultiplier tubes. These have in front of them a layer of hexagonal "concentrators" which allow the coverage of the photomultipliers to exceed $90 \%$. The surface of the array is approximately toroidal such that the path of the Čerenkov photons in water is $1.17 \mathrm{~m}$ irrespective of angle. The photomultiplier tubes need to be shielded from the magnetic field of $B A B A R$, and this is achieved by steel shielding around the water chamber and a bucking coil mounted near to the beam line. The overall single photoelectron resolution is approximately $8 \mathrm{mr}$, which, given that the average number of photoelectrons (assuming a 25\% quantum efficiency for the photomultipliers) varies between 25 and 50 depending on polar angle, accomplishes the required resolution on $\theta_{c}$. 


\begin{tabular}{|c|c|}
\hline Radiation length & $1.86 \mathrm{~cm}$ \\
Density & $4.51 \mathrm{~g} \mathrm{~cm}^{-3}$ \\
Light yield & $50-60 \mathrm{k}$ photons/MeV \\
Emission peak & $565 \mathrm{~nm}$ \\
Time constant & $940 \mathrm{~ns}$ \\
\hline
\end{tabular}

Table 2-6. Properties of crystal CsI(TI) scintillator.

\subsubsection{The Electromagnetic Calorimeter}

The average photon multiplicity in generic $B$ decays is 5.5 and, at PEP-II, $50 \%$ of these will have an energy below $200 \mathrm{MeV}[50]$. As a result, the $B$ reconstruction efficiency falls off very steeply with the minimum reconstructable photon energy. In addition, many $C P$ eigenstates have a $\pi$ in their final state, and these need to be reconstructed with high efficiency since the branching ratios for such decays are in general small. Even in the most kinematically extreme case, in the decay $B^{0} \rightarrow \pi^{0} \pi^{0}$, the mass resolution on the $\pi^{0}$ is dominated by the energy resolution rather than the angular resolution. These facts give rise to the need for a highly efficient electromagnetic calorimeter with very good energy resolution.

A material fulfilling these requirements is Thallium doped Caesium Iodide, which has been used successfully for many years at CLEO-II[51]. The relevant properties of $\mathrm{CsI}(\mathrm{Tl})$ are listed in table2-6. The target resolution, excluding the effects of electronics noise and machine background, is (for an energy $E$ in $\mathrm{GeV}$ )

$$
\frac{\sigma_{E}}{E}=\frac{1 \%}{\sqrt[4]{E}} \oplus 1.2 \%
$$

the constant term arising from individual crystal calibration errors, crystal non-uniformity and leakage from the front and rear of the crystal.

The structure of the calorimeter is made up of two parts - the barrel and the forward endcap. There is no rear endcap in the design due to the asymmetry of the machine and the consequently small solid angle that such a device would cover. The barrel consists of 48 rows in theta which contain 120 crystals in phi. These are arranged into modules containing 7 crystals in theta and 3 in phi. The length of the crystals varies between $29.76 \mathrm{~cm}\left(16.1 \mathrm{X}_{0}\right)$ at the rear, increasing to $32.55 \mathrm{~cm}$ $\left(17.6 \mathrm{X}_{0}\right)$ in the forward direction. The inner radius of the barrel is $91 \mathrm{~cm}$. The endcap has a conical section, with the front faces of the crystals at an angle 22.7 from vertical. The endcap consists of 8 rings arranged in modules to give a 20-fold symmetry in $\phi$. The three rows closest to the barrel have 120 crystals in phi, the next three have 100 and the inner two 80 . This gives a total of 6580 crystals. A further ring of the same dimensions as the last ring of crystals is filled with lead to provide shielding from machine backgrounds. All the crystals in the end cap have length $32.55 \mathrm{~cm}\left(17.6 \mathrm{X}_{0}\right)$ except those in the inner two rings which are shorter by $1 \mathrm{X}_{0}$ due to space limitations. The calorimeter extends $2.30 \mathrm{~m}$ in the forward direction, and $-1.56 \mathrm{~m}$ in the backward 
direction, covering $-0.938<\cos \theta_{C M}<0.895$. A cross section of the calorimeter can be found in figure 2-8.

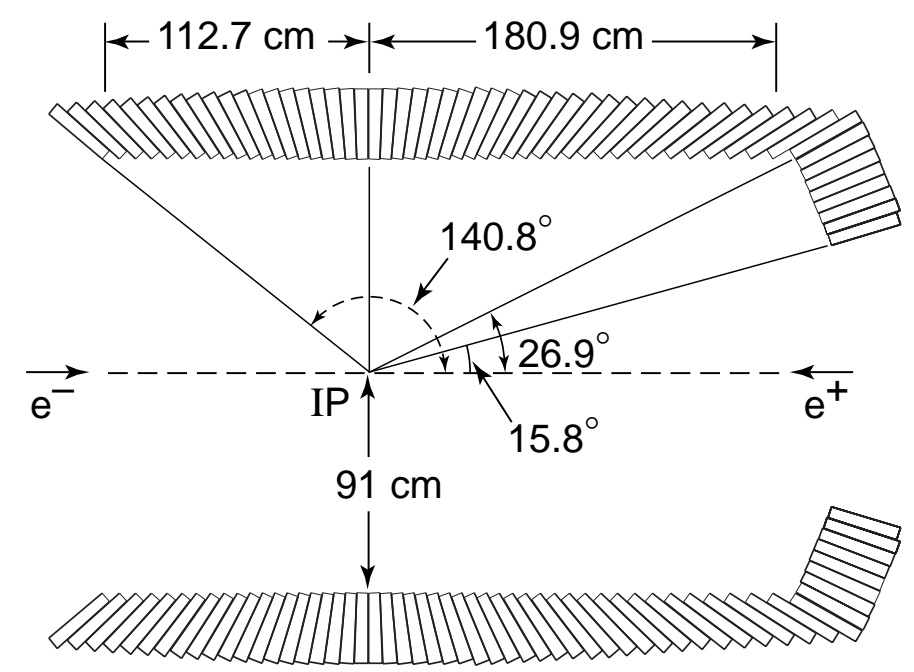

Figure 2-8. Cross sectional view of the electromagnetic calorimeter showing angular coverage.

The electron beam has the higher energy of the two.

The crystals are wrapped in a white diffuse reflector (Tyvek 1056D) and a layer of aluminium foil which gives RF shielding. Combined with the carbon fibre support structure of thickness $300 \mu \mathrm{m}$, this gives a $1.25 \mathrm{~mm}$ gap between crystals. To decrease the probability of a photon striking dead material, the crystals are arranged in a non-projective geometry in theta. The non projectivity ranges from $15 \mathrm{mr}$ in the central barrel to $45 \mathrm{mr}$ at the edges of the endcap. The crystals forward of the IP point forward, those to the rear point backward. In front of the crystals there is a double layered cylinder constructed of $1 \mathrm{~mm}$ thick which has a core layer of foam. This is to provide a gas seal necessary for humidity control and RF shielding.

The electronics and data acquisition system for the EMC is described in chapter 3. The projected performance is discussed in chapters 4 and 5 .

\subsubsection{The Instrumented Flux Return}

The iron flux return on the outside of the $B A B A R$ detector is instrumented with resistive plate chambers (RPCs) in order to provide a means of $\mu$ and $K_{L}^{0}$ identification and to use the high timing resolution on such devices to provide information on the beam crossing time. The novel aspect of the $B A B A R$ IFR is that it is graded, with increasing thickness of iron from the inside moving outward, providing a lower momentum reach for particle identification. 


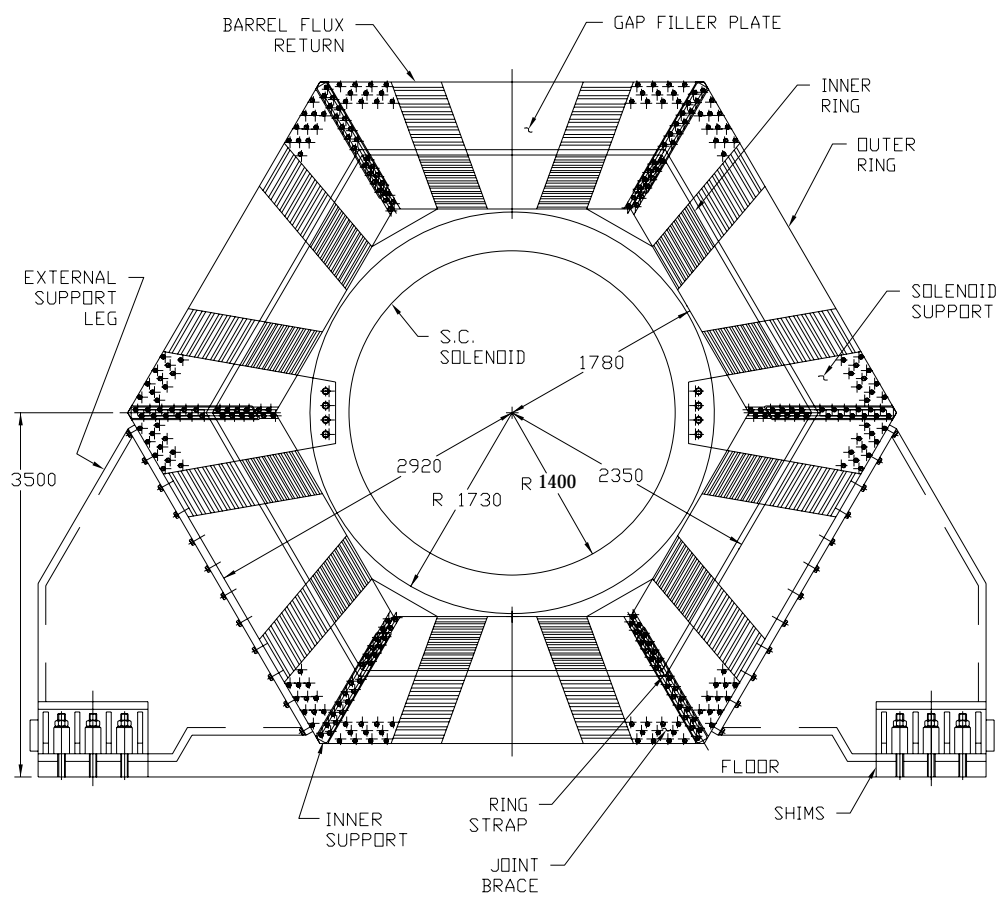

Figure 2-9. End view of the barrel section of the instrumented flux return, showing integration with the solenoid. The inner resistive plate chamber (iRPC) is not shown.

The three main sections of the IFR are the barrel, the endcaps and the inner RPC situated around the solenoid.

The barrel consists of 17 active layers situated between 18 plates of iron. The inner 9 plates are $2 \mathrm{~cm}$ in thickness, then 4 plates of $3 \mathrm{~cm}, 3$ plates of $5 \mathrm{~cm}$ and 2 of $10 \mathrm{~cm}$. The barrel is fabricated in sextants, and these are broken up into modules $125 \mathrm{~cm}$ wide and $181-320 \mathrm{~cm}$ long with increasing radius. An additional active layer is situated on the outside of the iron. An end view of the barrel can be seen in figure 2-9.

The endcap is hexagonal in cross section, and is split vertically to allow access to the inner detectors. The grading of the iron is similar to that of the endcap, excepting that the outer two layers are $5 \mathrm{~cm}$ and $10 \mathrm{~cm}$ in thickness. The gaps between layers in both the endcap and the barrel are $3.2 \mathrm{~cm}$.

The inner RPC consists of two cylindrical layers which surround the electromagnetic calorimeter, and an additional planar layer between the solenoid and the first layer of iron. One of the functions of the inner RPC is to provide information on particles which lose most of their momentum in the calorimeter and consequently do not reach the first iron layer. 


\subsubsection{RPC construction and readout}

The principle of operation of a resistive plate chamber is described in [52] and has been used in many experiments [53]. A cross sectional view of the $B A B A R$ RPC is shown in figure 2-10. On the outside are grounded aluminium sheets $40 \mu \mathrm{m}$ thick, which are insulated from the rest of the device by a layer of mylar. The pickup strips, also aluminium, are separated from the graphite coated Bakelite sheets by a $300 \mu \mathrm{m}$ layer of PVC. The $2 \mathrm{~mm}$ gap between the sheets is filled with an $\mathrm{Ar}: \mathrm{C}_{2} \mathrm{H}_{2} \mathrm{~F}_{4}: \mathrm{C}_{4} \mathrm{H}_{10}$ mixture which is held in an $8 \mathrm{kV}$ potential.

The barrel layers are filled with 3 RPC modules which are divided in $z$, while the endcap is divided vertically to house 2 modules. The cylindrical inner RPC is divided into 4 quarter cylinders, each with a radius of $147 \mathrm{~cm}$.

The readout strips on each side of the RPC are orthogonal to one another, and this, in conjunction with the finite vertical height, gives 3 dimensional position information.

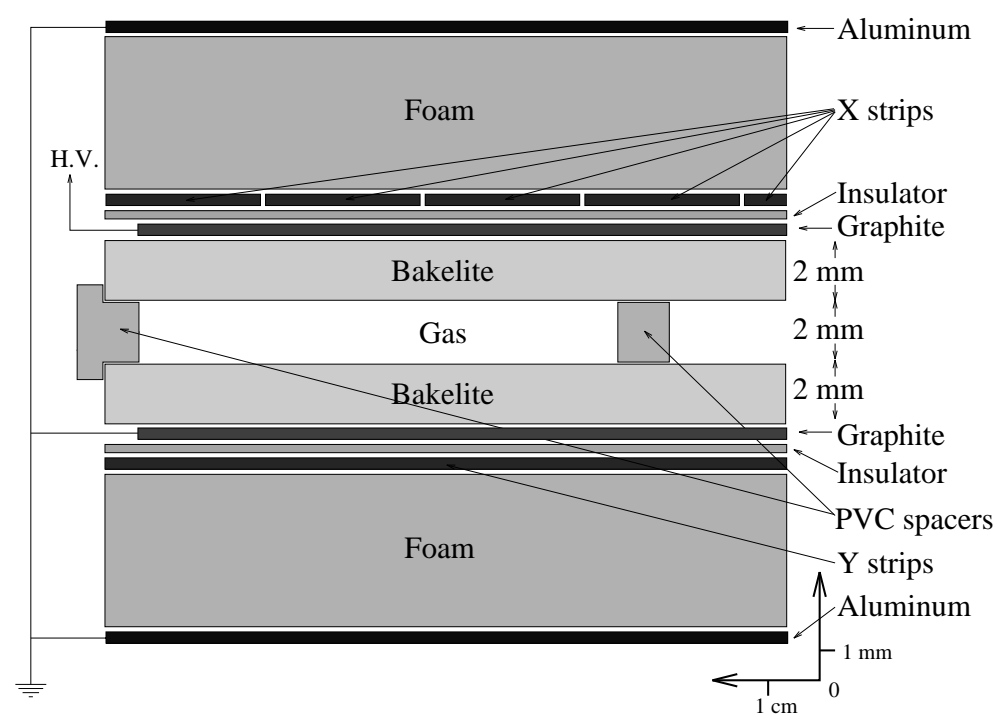

Figure 2-10. Cross section of the layout of a typical RPC used in BABAR's instrumented flux return.

The readout strips on the RPCs have a pitch of $38.5 \mathrm{~mm}$ in $z$ and varying between $21.7 \mathrm{~mm}$ to $33.5 \mathrm{~mm}$ in $\phi$ in the barrel, and $28.4 \mathrm{~mm}$ in the horizontal direction and $38 \mathrm{~mm}$ in the vertical in the endcaps. 16 such strips are passed into a front end card (FEC) which passes the active strips through TDC circuits which give a $1 \mathrm{~ns}$ resolution. The fast rise time (2-4ns) and the large signal $(300 \mathrm{mV})$ of the RPCs means that relatively simple fixed threshold electronics can be employed. The output of 64 such TDCs is stored, 1 bit per strip, in a buffer designed to allow for the trigger latency. The output of the buffers is then passed along an optical fibre to the BABAR DAQ system. 


\begin{tabular}{|c|c|c|}
\hline Physics sample & Minimum efficiency rate & Tolerable error \\
\hline$\Upsilon(4 \mathrm{~S}) \rightarrow B \bar{B} \rightarrow C P$ channels & $99 \%$ & $0.5 \%$ \\
$\Upsilon(4 \mathrm{~S}) \rightarrow B \bar{B} \rightarrow$ non- $C P$ channels & $99 \%$ & $0.5 \%$ \\
$B$ physics at the $\Upsilon(5 \mathrm{~S})$ & $99 \%$ & $0.5 \%$ \\
Charm physics & $95 \%$ & $2 \%$ \\
$q \bar{q}$ & $95 \%$ & $2 \%$ \\
$\tau$ physics: rare decays & $90 \%$ & $10 \%$ \\
$\tau$ physics: asymmetries & $95 \%$ & $0.5 \%$ \\
$\tau$ physics: branching ratios & $95 \%$ & $0.1 \%$ \\
$\gamma \gamma$ physics: exclusive meson pairs & $90 \%$ & $3 \%$ \\
$\gamma \gamma$ physics: all neutrals & $80 \%$ & $5 \%$ \\
$\gamma \gamma$ physics: exotic resonances & $90 \%$ & $4 \%$ \\
$\gamma \gamma$ physics: tagged & $90 \%$ & $5 \%$ \\
\hline
\end{tabular}

Table 2-7. Trigger efficiency for various physics channels to be studied with the BABAR detector.

\subsubsection{Trigger}

The trigger system for $B_{A} B_{A R}$ is required to deliver a near perfect efficiency for interesting physics events in demanding conditions, most notably imposed by the machine background. The requirements on efficiency are discussed in detail in [54] and are summarised in table 2.3.6. The total trigger rate must not exceed that allowed by the data acquisition system bandwidth $(2 \mathrm{kHz})$ in hardware and finally the maximum storage rate $(100 \mathrm{~Hz})$ in software. To do this, the trigger is split into two levels, Level 1 (hardware) which fulfills the initial rate reduction requirement, and Level 3 (software) which fulfills the second. In addition, the overall latency in Level 1 must not exceed $12 \mu \mathrm{s}$ with a jitter of no more than $1 \mu \mathrm{s}$.

The level 1 trigger consists of three parts: the drift chamber trigger, the calorimeter trigger and the global trigger. The calorimeter and drift chamber triggers construct elementary trigger objects, which are then combined in various ways by the global trigger to produce 24 trigger lines. The elementary trigger objects, which are somewhat simpler than the fully reconstructed objects for the two subsystems, are described in detail in [55]. The main objects are long tracks (with $p_{\mathrm{t}}>180 \mathrm{MeV} / \mathrm{c}$ ) which encompass the full extent of the drift chamber, short tracks (with $p_{\mathrm{t}}>120 \mathrm{MeV} / \mathrm{c}$ ), and calorimeter trigger towers, which consist of the sums in energy of a 3 crystal strip in phi along the length of the calorimeter. A level 1 accept signal is generated and sent to the data acquisition systems of the subdetectors if a pre-determined selection of trigger lines is set off for a given event. This selection can be related to physics requirements, or be set to 
allow calibration or monitoring runs. The level 1 accept signal must be sent to the subsystems with the latency and jitter specifications described above. At nominal machine background, the level 1 trigger is expected to pass events at $1.5 \mathrm{kHz}$. At ten times nominal, this is expected to be $16 \mathrm{kHz}$. Additional requirements looking at the separation of tracks or an increase in the minimum energy required for a calorimeter cluster have been shown to reduce this rate to an acceptable level.

The level 3 trigger is implemented purely in software, employed to reduce the level 1 rate of a maximum of $2 \mathrm{kHz}$ to the allowed event storage rate of $100 \mathrm{~Hz}$. The algorithms employed are a mixture of vertex cuts to eliminate events whose origin is far from the interaction point, event time cuts which eliminate events from bunch crossings either side of the physics event crossing and drift chamber to silicon vertex tracker track matching which helps reduce "events" from machine background. Bhabha and two photon physics events, whose expected rate at design luminosity totals to $177 \mathrm{~Hz}$, undergo pre-scaling in level 3 in order to keep the total rate down to the prescribed level.

If the machine background conditions are such that the level 1 accept rate exceeds $2 \mathrm{kHz}$, then the construction of a level 2 trigger to filter this rate down to an acceptable one for level 3 will be necessary.

The present performance of the trigger[55] indicates performance well within the criteria, with the exception of the efficiency for the one pronged $\tau$ decay at 10 times nominal background, which is at $92 \%$, instead of the required $95 \%$. Improvements are expected to this figure.

\subsection{Chapter Summary}

The measurement of $C P$ violation in the $B$ meson system requires advances in both storage ring and detector performance. The PEP-II storage ring has been described. The key aspects of the machine are the high design luminosity of $3 \times 10^{33} \mathrm{~cm}^{-2} \mathrm{~s}^{-1}$ and the asymmetry, colliding $9.0 \mathrm{GeV}$ electrons with $3.1 \mathrm{GeV}$ positrons. The impact of the machine on the design of the detector and the expected backgrounds generated by the machine have been described. An outline of the $B A B A R$ experiment has been given, describing the construction and readout of the five key detector elements. The readout and performance of the calorimeter is discussed in more detail in subsequent chapters. 


\section{Software for the Electromagnetic}

\section{Calorimeter}

\subsection{Introduction}

The reconstruction and simulation software is a key component in the operation of any experiment. This chapter describes the motivation for, and the key concepts of, the chosen technology used in the $B A B A R$ software environment. A description of the application of this technology in the aspects of calorimeter electronics simulation, reconstruction, particle identification and calibration will be given, along with an indication of the key components of the operation of these systems. The development and performance of these systems will be discussed in chapter 4 .

\subsection{C++ and object oriented design}

$B A B A R$ has chosen $\mathrm{C}++$ as its primary programming language. The principal reason for this choice is the ability to employ object-oriented programming techniques which allow for greater flexibility and control of large scale projects $\left(\mathcal{O}\left(10^{6}\right)\right.$ lines of code or more). In this section an introduction to the concept of object oriented programming will be given along with a summary of its key components. Sections 3.4.1, 3.4.3 and 3.4.4 will give examples of the employment of this technique in the calorimeter software.

\subsubsection{The Concept of an Object}

Procedural programming languages allow for the separation of functionality, but do not allow for the separation of what a function does from how it does it. In addition, the implementation of a given function is intrinsically linked to the data that the function uses. This leads to two problems. Firstly, the data in a function are "open" in the sense that operations on it are permitted by any function which uses that data. This can lead to functions corrupting the data which are sent to them which then has knock on effects for the rest of the system. Secondly, changes in the implementation of a function can affect directly the clients of that function, such that a small change in one function can result in a large number of changes throughout the system in order to 
accommodate the new implementation. This problem is increasingly intractable as the scale of the project increases. With $B A B A R$ 's reconstruction and analysis software extending to over a million lines of code, new techniques to control this problem have been employed.

In object oriented programming, the fundamental unit of construction is the object, not the procedure. An object is a set of data and the functions that can be performed on that data. The definition of such an object is referred to as a class-the word object is reserved for particular instances of a class within a program. In general, the representation of the data is hidden from the client, and one may only ask for values of the data, or the result of calculations on it. For example, an electromagnetic cluster can be thought of as an object. The data in this object are the crystals which make up the cluster and their energies. However, all the properties of a cluster can be passed to client code without ever having to give the client access to the crystal energiesclients need not know about the data representation. This has two advantages: firstly that the integrity of the data can be guaranteed, and secondly that the external clients can code in terms of the questions they need to ask - the interface of the object-and are then immune to any changes in the data representation. These ideas are developed further in two key concepts: abstraction and encapsulation.

\subsubsection{Abstraction}

Abstraction is the separation of the external view of an object from its implementation. Booch[56] describes it as follows:

An abstraction denotes the essential characteristics of an object that distinguish it from all other kinds of objects and thus provide crisply defined conceptual boundaries, relative to the perspective of the user.

Clients coding in terms of abstractions do not concern themselves with either the data that an object uses in its implementation, nor the particular implementation itself. This allows for either of these to change without that change propagating to client code, resulting in much reduced complexity.

The mechanism in $\mathrm{C}++$ that allows for abstraction is inheritance. If a class inherits from another class, then the function definitions (and in certain cases some data) in the so-called base class can be used by the inheriting class (called the derivative) or, more usefully, be overridden by that class. In this way, alternative implementations of a given function are made available to clients, who can code in terms of the base class (the abstraction) but receive a given implementation by being given a particular derivative. For example, a track matching algorithm is concerned with the location of an energy deposit in the calorimeter. That energy deposit can be described by either collections of raw crystal energies, crystal energies which have been shared among several clusters, or calibrated crystal energies. The abstraction is "some object which knows how to tell you its position," and the three cases described provide differing implementations. A pure abstraction is one that provides no default implementation whatever, and leaves all the details up to derivative classes. 


\subsubsection{Encapsulation}

Encapsulation is the process of separating the implementation of a given class from its interface. As described above, the interface defines those questions that a client may ask of an object about which the client can make assumptions. The implementation of a class is the part about which clients may not make assumptions. The most usual form of encapsulation is data hiding which prevents clients from directly accessing the data inside an object. This not only preserves data integrity, but allows for the internal representation of an object to change without affecting external clients. Booch's definition is:

Encapsulation is the process of compartmentalising the elements of an abstraction that constitute its structure and behaviour; encapsulation serves to separate the contractual interface of an abstraction and its implementation.

The $\mathrm{C}++$ mechanism that allows for data hiding is the concept of private and public members of a class. The public members are those functions (and data, though this is not good practice) which are visible to external clients. Private functions and data are not visible to external clients and so are free to change without knock on effects in other code.

\subsubsection{Object Oriented Design}

The use of objects in programming brings in the advantages discussed above, but is only fully exploited when combined with object oriented design. This allows for an expression of the relationships between classes to be developed, before code is written, which defines the logical relations amongst the classes, usually in the form of a class diagram. Examples of class diagrams and notation are given in appendix B. In general the goal is to ensure that objects depend on abstractions, and not on the details of implementation. This has the effect of reducing couplingthe amount of changes in client code necessary to accommodate a change in implementationreducing maintenance and increasing reliability.

Certain constructs in object oriented design have a sufficiently large number of applications that they have become "standard" designs, known as patterns. These are collections of objects that solve a particular design problem, in much the same way as there are electronic circuits that solve particular engineering problems. A detailed discussion of the merits of patterns will not be given here. For further details, see [57].

\subsubsection{Relevance to physics capability}

The rapid acquisition of a detailed understanding of the $B A B A R$ detector will be crucial to the operational success of the experiment. Furthermore, the limitations of even detailed Monte Carlo 
studies mean that changes in algorithms and consequent physics capability can be expected at a high rate during the collection of first data, and this must be allowed for in the software design. Two aspects of properly employed object oriented design are beneficial in this regard. Firstly, a system depending on abstractions (termed "loosely coupled") results in less maintenance and a more reliable structure, with the obvious benefits for physics development. A more tangible aspect is that of algorithmic abstraction, whereby the fundamental aspects of an algorithm are abstracted and encapsulated, and a particular implementation providing improved physics capability can be readily incorporated into the rest of the software with the minimum of effort, making both comparisons and physics optimisation easier.

The philosophy of providing a well defined framework for the calorimeter reconstruction software has concentrated on providing a core set of functionality with as much abstraction as possible, such that an operational system is available now, but it is a system that is easily extensible in the advent of better physics capability and understanding.

\subsubsection{The $B_{A} B_{A R}$ Framework Software}

The BABAR Framework, now a joint collaboration between $B A B A R$, CDF and CLEO, provides the core operation of the reconstruction, analysis and electronics simulation software. It is described in detail elsewhere[62]. A summary of the key components is given here.

The principle building blocks of the Framework are the Event, the Environment and the Module.

The Event The Event is an object consisting of the collection of all data relating to a particular physics event. These are usually organised in terms of lists of objects (e.g. clusters, tracks, particle candidates) but may consist of more complicated objects such as associations between items in different lists. The ProxyDict mechanism[68] is employed to allow extensibility of the Event to new data types as these become necessary. No data type in the Event is required to know about any other.

The Environment The Environment is an object similar in concept to the Event, but relates to all the data which are necessary to process the physics data - geometry, calibration constants, conditions data.

Framework Modules A Framework Module is a high level abstraction that provides a particular piece of event data processing. In addition, tools for run time configuration are provided. The usual mode of operation for a subclass of a module is to extract the necessary data from the Event/Environment that it needs to do its processing, and return its results to the Event. For example, a cluster finding Module will retrieve the crystal energies from the Event, use the Environment to obtain the geometrical information necessary for clustering, and return the cluster objects to the Event. 
Framework Modules are configured into the executable in Sequences designed to produce a more general task such as the calorimeter reconstruction. Sequences and individual modules can, once compiled into an executable, be configured into and out of it at run time.

\subsection{Simulation software}

The central simulation software for BABAR is the GEANT3 [45] based BBSIM program. This simulation provides, using an input of four vectors and particle types of the decay products of the $\Upsilon(4 \mathrm{~S})$ and a geometrical description of the detector material, an output list of energy deposits in the active volumes. The process of digitisation is not part of this simulation and is coded in $\mathrm{C}++$. It is this part of the simulation for the calorimeter which is now described.

\subsubsection{The calorimeter electronics simulation}

A detailed description of the calorimeter electronics design can be found elsewhere [61]. A brief summary of the essential points is as follows. The scintillation light from the crystal is picked up by two $(1 \mathrm{~cm} \times 2 \mathrm{~cm})$ photodiodes mounted directly on the rear face. The output from each photodiode is sent through a pre-amplification and $C R-R C^{2}$ shaping integrated circuit, mounted close to the photodiodes in order to reduce pickup noise. The shaping times are $0.8 \mu \mathrm{s}$ (differentiation time) and $0.25 \mu \mathrm{s}$ (integration time). The output from the shaping circuit is sent through channels of gain $\times 1$ and $\times 32$ to the CARE (Custom Analogue Range Encoder) chip before being encoded by a 10 bit ADC. The CARE encodes the incoming data in the following gain ranges: $1(1 \times 1), 4(1 \times 4), 32(32 \times 1)$ and $256(32 \times 8)$. In this way, the output of the CARE is a 12 bit number (a 10 bit mantissa and a two bit range indicator) but the dynamic range represented is 18 bits. The ADC operates at $3.7 \mathrm{MHz}, 1 / 64$ of the $B_{A} B_{A R}$ beam crossing clock rate. The output of the ADC, plus 2 bits encoding the range of the CARE, is passed over an optical fibre to the data acquisition, where a sophisticated read out process, referred to as feature extraction, takes place. This is described in more detail in section 3.3.2. The output is also passed to the trigger systems. The bandwidth of the data acquisition system limits the number of crystals that may be feature extracted at the maximum level one trigger rate $(2 \mathrm{kHz})$ to approximately half of the calorimeter crystals. The result of the trigger determines which crystals are feature extracted. If a given crystal's analogue energy determined by a simple search in the bins around the trigger time, taking into account the rise time of the shaping response, is above a threshold (this will be background dependent, but is around $10 \mathrm{MeV}$ ) then that crystal's ADC output is passed for feature extraction, as are those of neighbouring crystals, or neighbouring strips of crystals if the crystal is at the edge of a trigger tower[61]. The feature extraction uses a matched digital filter which is described in section 3.3.2.

The input and output objects of the front end electronics simulation are the EmcGHit and the EmcDigi respectively. 


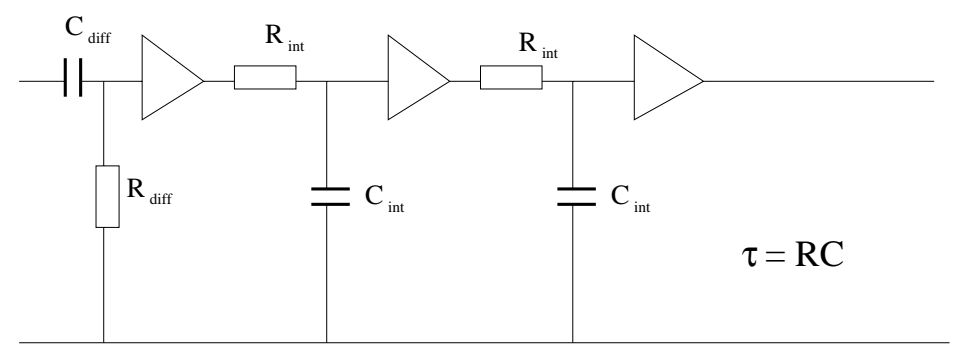

Figure 3-1. $\quad C R-R C-R C\left(C R-R C^{2}\right)$ shaping circuit for the photodiode readout. The differentiation $(C R)$ stage has a time constant of $0.8 \mu \mathrm{s}$ and the integration $(R C)$ stages have identical time constants of $0.25 \mu \mathrm{s}$. The input to this circuit is the amplified photodiode output. The output from this circuit is fed to the ADC circuit.

EmcGHit This class contains the Monte Carlo truth information for a particular crystal. The deposited energy, the time of the hit, and crystal index information are all provided.

EmcDigi This class represents the front end electronics output for the calorimeter. Access to the energy (both in raw integer format and in $\mathrm{GeV}$ ) and time are provided, along with a channel identifier which can be converted into a theta and phi crystal index.

The simulation for the front end electronics was written to convert the input from the detector simulation (a list of EmcGHits) into the output (a list of EmcDigis). To this end, the following classes were written:

EmcWaveform This class encapsulates the properties of the crystal and the electronics up to and including the ADC. The crystal properties are those of the exponential decay constant of the light output and the number of photons created inside the crystal. This number is folded into the quantum efficiency of the photodiode to give a number of photoelectrons observed per $\mathrm{MeV}$ of energy deposited. It is envisaged that this number will come from measurements of crystal properties, but at present is set at 5000 p.e. per MeV. The exponential decay constant is $900 \mathrm{~ns}$ for Thallium doped CsI. The shaping and preamp electronics are described in an analytic formula calculated by forming an expression for the various components in the Laplace domain, multiplying and applying the inverse transform. The preamp is assumed to have an infinite time constant (the time constant is typically 1000 times the other constants in the system) which in the Laplace domain gives a $1 / s$ term. The crystal light collection constant is an exponential decay, giving a $1 /(s+a)$ term, where $a$ is the reciprocal of the decay time constant. The shaping circuitry is shown in figure 3-1. The differentiation stage gives a $s /(s+b)$ term $(b=1 / C R)$, and the integration parts of the circuit, which have the same time constant, again give exponential terms, giving a $1 /(s+c)^{2}$ contribution $(c=1 / R C)$. This gives the final expression

$$
\frac{1}{(s+a)(s+b)(s+c)^{2}} \text {. }
$$


The response function, then, of the entire system is given by

$$
\begin{aligned}
h(t)= & \frac{1}{(a-b)(a-c)(b-c)}\left[e^{-c t}(a-b)\left(t-\frac{1}{a-c}-\frac{1}{b-c}\right)\right. \\
& \left.+e^{-b t} \frac{(a-c)}{(b-c)}-e^{-a t} \frac{(b-c)}{(a-c)}\right] .
\end{aligned}
$$

The waveform class has the additional functions of digitising its contents when given the least significant bit value, and the addition of Gaussian electronics noise when given the noise width energy equivalent. Strictly, random noise should be passed through the shaping circuitry response function before being added to the waveform. However this is expensive in CPU terms since this must be done for each sample of each waveform for all 6580 crystals. In addition, the noise on a given crystal's output is dominated by machine background (see section 3.3.2.2) and so electronics noise was simulated by overlaying Gaussian noise on the already shaped waveform.

Feature extraction can also be performed in the Waveform either using a standard maxima search method or by using a digital filter (see section 3.3.2).

EmcGHitsToWaveform A Framework module derivative. This module receives the simulated energy deposits from the event (the GHits), creates the necessary Waveforms for each crystal, passes the GHits to the appropriate Waveforms such that the response may be calculated, and places a table of the resulting waveforms in the event. This module has control over the parameters given to the Waveforms (electronics time constants, photoelectron efficiency, crystal time constants), such that these may be varied either for simulation purposes or as a result of detector measurements.

EmcSparsify Also a Framework module, responsible for stripping the event waveform table of those waveforms that do not pass the sparsification algorithm. The present implementation is one that takes all the neighbouring crystals of those crystals that pass the analogue threshold, and does not take into account the strips associated with the edge of trigger towers.

EmcWaveformsToDigis The final Framework module in the electronics simulation sequence, this is responsible for feature extraction. The standard calorimeter feature extraction employs a matched digital filter. The module consequently has two functions: one is to construct the filter response function, and the other is to perform feature extraction on the waveforms passed to it in order to extract energy and timing information. The filter function is constructed from the noise power spectrum and knowledge of the ideal signal (see below.) The noise power spectrum is determined by a windowed power spectral analysis[63] performed on all the waveforms in an "event" which contains only machine background. The ideal signal is calculated analytically. Once the filter function is constructed, it is convoluted with each waveform to be feature extracted in turn, and the energy and timing information extracted by interpolation around the observed maxima.

The above structure allows the table of waveforms for one event to be given to the level 1 trigger simulation. The trigger then provides the time of the event and it is this that is used to provide the correct location in the waveform to do feature extraction. 


\subsubsection{Digital filtering}

The machine background rate at PEP-II is such that at ten times nominal background, the calorimeter crystals will each receive about $400-500 \mathrm{keV}$ of soft photons per microsecond (see figure 3-2). Even at nominal background, the employment of a long shaping time in order to reduce electronics
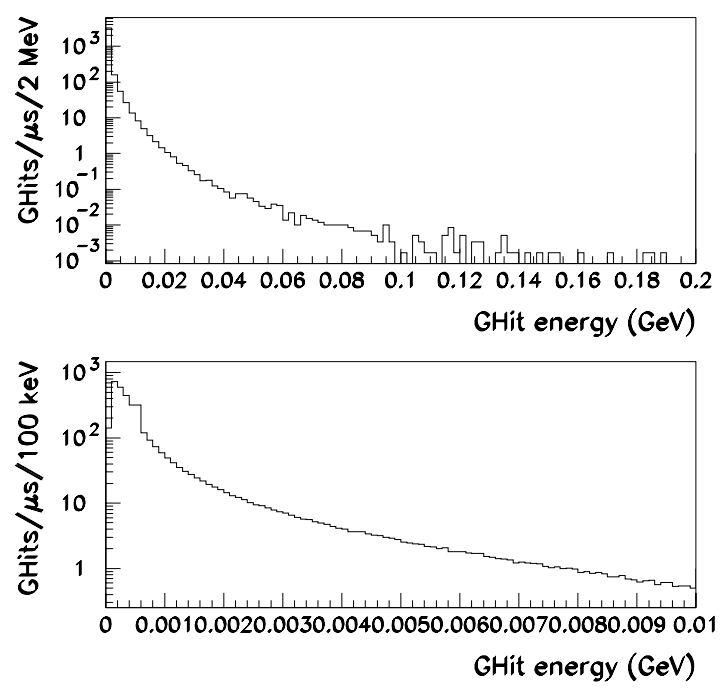

Figure 3-2. Energy deposit in the calorimeter at times ten nominal machine background, summed over all crystals.

noise will result in the overlap of many background photons on a given signal photon, which will degrade the resolution. The use of a short shaping time will decrease the probability of overlap, but will increase electronics noise. The solution implemented for $B A B A R$ is to pass the ADC output through a matched digital filter in order to maximise signal to noise. The theory of this filter will now be described, and its impact discussed in section 3.3.2.2.

\subsubsection{Theory of the Matched Filter}

Let us consider the case in which a signal $A(i \omega)$ passes through a filter with response function $R(i \omega)$ which is to be optimised to maximise $S / N$ for a noise input of spectral power density 
$N(i \omega)$. For this case, the signal to noise ratio is given by

$$
\frac{S}{N}=\frac{1}{2 \pi} \frac{\left|\int_{-\infty}^{+\infty} A(i \omega) R(i \omega) d \omega\right|^{2}}{\int_{-\infty}^{+\infty} N(i \omega)|R(i \omega)|^{2} d \omega}
$$

The numerator can be written in the form

$$
\left|\int_{-\infty}^{+\infty} \frac{A(i \omega)}{[N(i \omega)]^{\frac{1}{2}}}[N(i \omega)]^{\frac{1}{2}} R(i \omega) d \omega\right|^{2}
$$

so then, by use of the Schwarz inequality,

$$
\begin{aligned}
\frac{S}{N} & \leq \frac{1}{2 \pi} \frac{\int_{-\infty}^{+\infty}|A(i \omega)|^{2}[N(i \omega)]^{-1} d \omega \int_{-\infty}^{+\infty} N(i \omega)|R(i \omega)|^{2} d \omega}{\int_{-\infty}^{+\infty} N(i \omega)|R(i \omega)|^{2} d \omega} \\
& =\frac{1}{2 \pi} \int_{-\infty}^{+\infty} \frac{|A(i \omega)|^{2}}{N(i \omega)} d \omega .
\end{aligned}
$$

If the signal to noise ratio is bounded above in this way, then it may be maximised by finding the response function $R(i \omega)$ which gives the equality in the above equation. It can be seen that by setting

$$
R(i \omega)=\frac{A(i \omega)^{*}}{N(i \omega)}
$$

the equality will be satisfied. Equation 3.6 must then be solved for $R$, which is matched to the signal $A$. A fuller discussion of the above may be found in [58]. Equation 3.6 becomes, in the time domain

$$
a(-t)=r(t) * n(t),
$$

where a lower case letter refers to the Fourier transform of the corresponding upper case variable. By definition (see [59] p.42), the power spectral density of a function in the frequency domain is the Fourier transform of the so-called auto-correlation function of that function in the time domain. The auto-correlation function $A$ for a discrete time function $f$ is given by

$$
A(m)=\sum_{n=0}^{n=N-1-m} f(n) f^{*}(n+m),
$$

subject to $A(-m)=A^{*}(m)$, where $N$ is the number of samples in the data set. If $f$ is real, as is the case for the noise function, then $A(m)=A(-m)$. As a result, the convolution described in equation 3.7 is given by

$$
\left(\begin{array}{cccc}
n(0) & n(1) & \cdots & n(M) \\
n(-1) & n(0) & \cdots & n(M-1) \\
\vdots & \vdots & & \vdots \\
n(-M) & n(-M+1) & \cdots & n(0)
\end{array}\right)\left(\begin{array}{c}
r(0) \\
r(1) \\
\vdots \\
r(M)
\end{array}\right)=\left(\begin{array}{c}
a(M) \\
a(M-1) \\
\vdots \\
a(0)
\end{array}\right)
$$




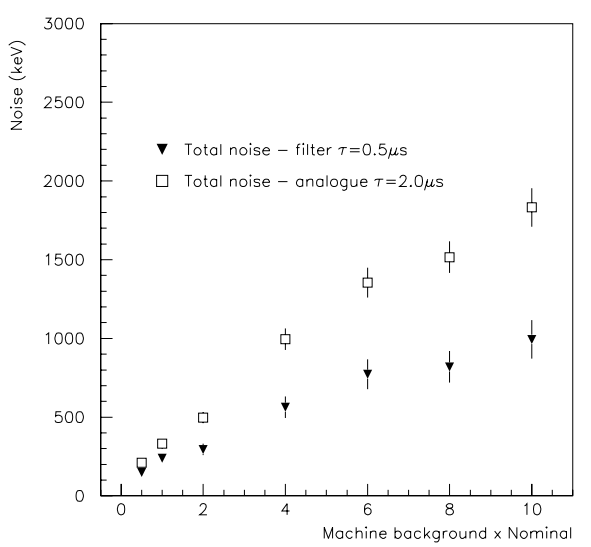

Figure 3-3. Individual crystal noise as function of machine background. The upper points represent a classical analogue readout with a shaping time of $2 \mu \mathrm{s}$ such as that used in CLEO. The lower points represent a digitally filtered readout using a short shaping time. It can be seen that the noise from soft photon machine background dominates over electronics noise (known to be about $150 \mathrm{keV})$.

where $M$ is the number of frequencies used in the discrete Fourier transform of the signal. Using the symmetry property of the auto-correlation function described above, this matrix equation becomes

$$
\left(\begin{array}{cccc}
n(0) & n(1) & \cdots & n(M) \\
n(1) & n(0) & \cdots & n(M-1) \\
\vdots & \vdots & & \vdots \\
n(M) & n(M-1) & \cdots & n(0)
\end{array}\right)\left(\begin{array}{c}
r(0) \\
r(1) \\
\vdots \\
r(M)
\end{array}\right)=\left(\begin{array}{c}
a(M) \\
a(M-1) \\
\vdots \\
a(0)
\end{array}\right)
$$

which may be inverted to give the required response function $r$. Since $r$ may be thought of as a weighting of the components of the convolution, the $M$ components of $r$ are referred to as weights. When the noise function is a mixture of machine background and electronics noise, the weights typically do not have significant structure beyond $M=16$. This allows for a reduction in the amount of computation required for feature extraction.

The ideal signal, $a$, is generated using knowledge of the pre-amp and shaping parameters, the analytic form for which is shown in equation 3.2. The noise auto-correlation function is calculated by taking the Fourier transform of the average power spectrum of the background. The background 
consists of time windows of several crystals' ADC output, which consist of the pre-amp/shaping response to the soft photon background hits, electronics noise and digitisation.

\subsubsection{Digital filter performance}
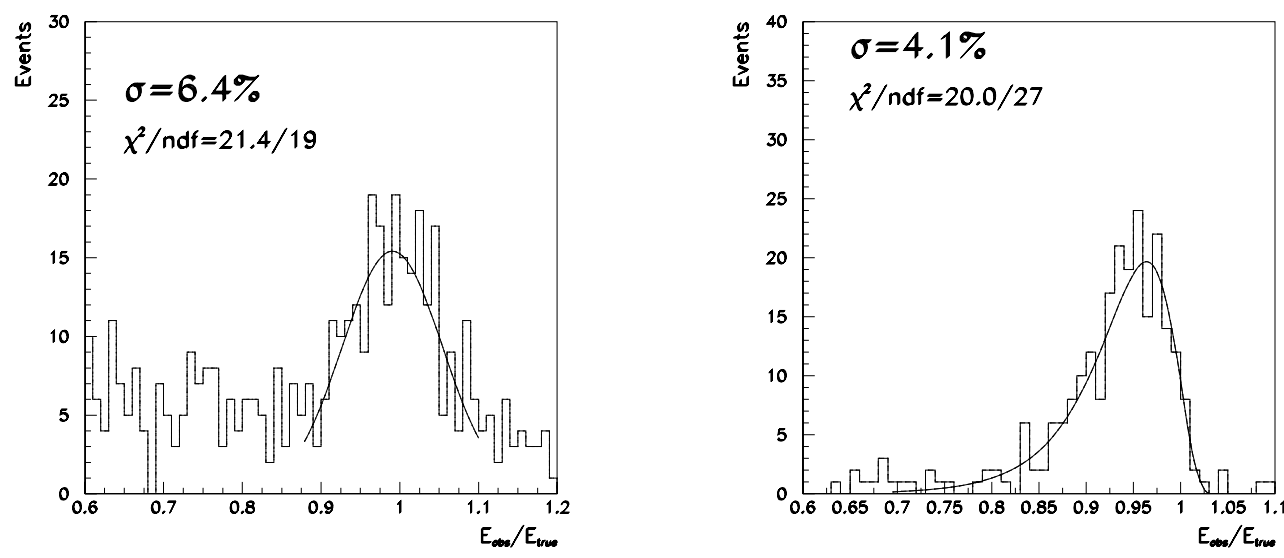

Figure 3-4. Resolution for $320 \mathrm{MeV}$ photons at times ten nominal background with classical feature extraction (left) and matched digital filter feature extraction (right).

There are two aspects of the performance of the digital filter that are closely linked but must be assessed separately. The first is the noise observed on a given crystal's readout. Here "noise" refers to machine background as well as electronics noise-indeed it is the latter which dominates. The average noise on individual crystals was estimated by observing the simulated noise width on a 20 $\mathrm{MeV}$ energy deposit made on each crystal. The results (averaged over all crystals) are shown in figure 3-3 where a comparison is made to the more usual technique of using a longer shaping time to average out the electronics noise. The other aspect is the resolution impact on energy deposits in the calorimeter and the number of spurious clusters which give rise to combinatoric background. In figure 3-4 the resolution for $320 \mathrm{MeV}$ photons is shown assuming background levels ten times the expected nominal. The quoted resolution is the full width at half maximum/2.36. It can be seen that a significant improvement is gained by using the filtered feature extraction, both in terms of resolution and in the number of "fake" clusters around the signal peak that originate from background. 


\subsection{Reconstruction software}

This section will describe the reconstruction chain in the calorimeter, from the creation of the most basic objects through to the association of particle hypotheses to individual energy deposits. At each stage, a discussion of some of the software engineering issues will be given in the context of their impact on the physics capability of the software.

\subsubsection{Basic reconstruction}

\subsubsection{Clustering}

The most basic task of reconstruction for a calorimeter is the identification of connected regions, or clusters. There are several ways to define these. One of the common methods is the definition of arrays of crystals (typically $3 \times 3$ or $5 \times 5$ ) in which some energy above a threshold is deposited. This is used for example in CLEO [60]. The endcap geometry in $B A B A R$, whilst not precluding this approach, means that such a definition is not available everywhere in the calorimeter. For this reason, the $B A B A R$ definition of a cluster is essentially one of a contiguous region of crystals above a certain threshold. A comparison of the two algorithms may be found in section 4.2.2.

\section{The clustering algorithm}

The algorithm employed to do the clustering is as follows[65]: the highest energy crystal is used as a seed. Its neighbours are then added to a list for potential inclusion in the cluster. If any of these neighbouring crystals are above a certain threshold, then their neighbours are added to the list and the neighbours of these new crystals are searched for crystals above threshold. This continues until no more of the neighbouring crystals are above this threshold. The list of crystals for potential inclusion is then traversed and only those crystals above a second threshold are included. If the cluster itself then has a total energy greater than a third threshold, it is kept.

\section{The EmcCluster class}

The EmcCluster class is designed to encapsulate the aspects of an energy deposit in the calorimeter. The principal interface provides information on the energy, position and error matrix associated with the cluster. Its implementation is in terms of a collection of crystal energies (the EmcDigi objects described in section 3.3.1 and in more detail in [66]), which allow for the above quantities to be calculated. The position information is determined by a center of gravity method. The energy is simply the sum of the individual crystal energies. The error matrix is filled at present by estimation of the errors from Monte Carlo.

Classes which use the crystal collection to provide information on the distribution of crystals within the cluster, and different estimations of the position of the cluster are also provided by the 
EmcCluster class. This allows for the extension of the number of quantities so calculated without having to recompile all external code which depends on this class.

EmcCluster is itself a subclass of AbsRecoCalo, a generic calorimetry class designed to provide a common interface between deposits in the EMC and the IFR. The AbsRecoCalo interface defines the pure virtual functions returning position, energy and error matrix, which are implemented as described above.

\subsubsection{Bump splitting}

The contiguous regions of crystals formed into clusters may well be the result of energy deposited by more than one particle if the angular separation of the particles is small. For this reason it is desirable to separate the clusters into regions containing one and only one local maxima. This is particularly true in high background conditions where the separation of the soft photon machine background from the physics data is important.

Two distinct operations are involved in this division: the location crystals which are potential maxima, and the sharing of the energies of the other crystals in the cluster among these local maxima such that each local maximum then becomes a cluster like object with a collection of crystals, with each crystal having some fraction of the total observed energy originally deposited in that crystal. These local maxima with collections of "shared" crystal energies are called "bumps".

As the primary purpose of the electromagnetic calorimeter is to measure the energies of photons and electrons, the optimisation of the two processes was done based on an electromagnetic hypothesis. It is recognised that hadronic showers produce showers with fluctuations which could potentially mimic the behaviour of an electromagnetic maxima and it is this causing of "fake" bumps that the optimisation is designed to minimise. The creation of bumps does not preclude the use of clusters in an analysis, as algorithms in the analysis software to ensure that the same energy deposit is not included twice. This ensures that hadronic showers (for which the entire cluster should be treated as one entity) can be used side by side with the electromagnetically divided bumps.

\section{The EmcBump class}

The EmcBump class is a derivative of EmcCluster. This has two implications - the first is that it is necessarily a derivative of AbsRecoCalo, and can consequently be used in all code implemented in terms of that interface. The second is that all the implementation of the EmcCluster class is available to the EmcBump class where it is appropriate to use it. The implementation of the EmcBump class in terms of "shared" crystal energies permits re-use of all of the code written to calculate quantities in the EmcCluster. This is permitted by the construction of EmcBump objects with objects of a subclass of EmcDigi, EmcSharedDigi which, when asked for its energy, returns the raw crystal energy multiplied by a weight whose value lies between 0 and 1 . The sum of weights for a given crystal is normalised to 1 . The determination of that weight is described below. 


\section{The Bump Finding and Splitting Algorithms}

The optimisation of the bump finding and splitting algorithm is not definitive and may be subject to change as the scale of the machine backgrounds becomes known, or if the purpose of the splitting changes: for example a given analysis may wish to optimise for looking for photons close to electron showers, whose profile will be different to that of two photons in close proximity because of the bending of the electron trajectory caused by the magnetic field. For this reason, it is desirable to preserve the ability of the module responsible for bump creation to be able to change the method of both maxima location and cluster splitting at runtime according the type of optimisation required. To this end, two abstractions are employed: firstly an abstract local maxima finder which takes a cluster as input and outputs a list of the crystal indices of the local maxima. Secondly an abstract bump splitter whose purpose is to take the list of local maxima, divide the cluster by the creation of the shared digis described above, and return a list of newly created bumps.

In this way, through the employment of a factory method [57], the Framework Module EmcMakeBump is able to select a given algorithm for location and splitting by using only the above abstractions, yet be configured with a particular instance of the algorithms from the factory according to a user specified string.

\subsubsection{Track-cluster matching}

The identification of charged and neutral particles is then undertaken after clusters have been isolated and, where appropriate, divided into electromagnetic maxima. The association of a given energy deposit to a track is not always unambiguous. Several cases complicate the process, for example hadronic showers which have a wide spatial distribution which result in a centroid some distance from the track impact point; bremsstrahlung from electrons which results in a mismeasurement of the track parameters; scattering in the DIRC which results in a different impact point in the EMC that would be expected from the track position at the end of the drift chamber. For this reason it was decided not to separate clusters into charged and neutral lists: moreover that the output for use in physics analysis should be associations between tracks and clusters at some confidence level. Further, it is envisaged that several algorithms for track matching will be developed over the course of the experiment. Consequently it is important that the output of these, and their interface to external code, is well defined. The classes used to achieve these goals will now be described.

\section{Association maps}

The matching between track and cluster at some confidence level necessitates a means of associating many sets of three objects together, each set consisting of a track object, a cluster object and 
a separate object containing information on the quality of the match between the two. Further, it is envisaged that it will be possible to match more than one track to a cluster, and possibly more than one cluster to a track. Thus there is a need to provide a three-way, many to many association table. A mechanism of $\mathrm{C}++$, known as templating, allows for such a table to be written independently of the objects that will go into the table. Thus, once written, the table can be used for other associations, such as that between Monte Carlo truth and reconstructed Monte Carlo particle candidates, or between EMC and IFR observations.

Two such maps were written: one providing a many to many association between the sets of three objects, but providing only one-directional queries, and one providing bi-directional queries.

\section{Track matching algorithms}

The abstraction of the mechanisms used to match tracks to clusters is achieved by a class, EmcAbsTrkMatchMethod which defines two methods, one for matching tracks to clusters, and one for the reverse operation. Subclasses of this one are responsible for implementing the details of the matching algorithms. The output of these classes is two association maps of the uni-directional type, matching tracks to clusters and vice versa. It has not yet been demonstrated that the flexibility of allowing different algorithms for the two different operations is necessary. An alternative option would be to define just one such method and use the bidirectional map. The Framework module, EmcTrackMatch, is allowed to choose between differing algorithms by the use of a factory method configurable by a run-time modifiable string parameter.

An original implementation looked within a $10 \mathrm{~cm}$ radius sphere around the impact point of the track for cluster centroids which were within this distance, using simple geometrical shapes to represent the inner surface of the calorimeter (cylinder for the barrel, cone for the endcap.) This has since been superseded after a detailed study [64], and the ease of incorporating the new algorithms serves as a demonstration of the flexibility of the design.

\subsubsection{Particle identification}

The calorimeter serves as the primary particle identification device for photons, $\pi^{0} \mathrm{~s}$ and electrons. It is also expected to provide information to cross system identification of minimum ionising particles (both muons and non-interacting charged pions) and neutral hadrons. Some preliminary techniques for photon and $\pi^{0}$ ID are discussed in chapter 4 . This section will discuss the software framework developed for the implementation of particle ID in the calorimeter. The emphasis has been on using object oriented design to allow for the increase in the number of particles that can be identified as detector knowledge improves as well as allowing for the improvement and replacement of algorithms as increasing sophistication becomes available.

\footnotetext{
${ }^{1}$ A separate object to contain the quality information allows for the extension of the information contained within such an object (typically the minimum information is a significance level for the match) to be extended without alterations to the cluster or track class.
} 
The class diagram for the particle ID design can be found in appendix B, figure B-3. The central components of the design are the EmcCand class, which encapsulates the concept of an observation or observations in the EMC which have been calibrated and have some particle identification information attached to them, an EmcPidInfo class which contains the information on the relative merits of the particular observed energy deposit(s) with respect to some particle hypothesis, the EmcCandidateMaker class which is responsible for constructing the EmcCands that may be appropriate to the hypothesis under consideration ( an example of this is the assembly of an EmcCand which consists of a Cluster or Bump plus a track as a possible electron candidate) and the EmcIdentifier class, an abstraction incorporating the concept of giving information about a given energy deposit under a certain particle hypothesis. These classes are now described in more detail.

\section{EmcCand}

The EmcCand class is a derivative of AbsRecoCalo. It contains one or more energy deposits in the form of EmcClusters (or derivatives) and also contains a place holder for a track object which may be empty. The usual functions of position, energy and error matrix access are overridden to provide calibrated forms of these quantities. The calibration is provided by implementations of the EmcAbsCalibrator class, discussed in section 3.4.4. Also provided is access to the EmcPidInfo object, discussed below. The primary source of EMC information provided to the BABAR analysis software is in terms of the calibrated EmcCands with particle identification information attached.

\section{EmcPidInfo}

The EmcPidInfo class allows for access to information regarding the compatibility of a given observation to the expected observation for a given particle hypothesis. There are three important aspects to this. The first is that the implementation places no restrictions on the number of hypotheses tested. Numerical results relating to the likeness of a particle with a given hypothesis are stored in a extensible dictionary of associations between the objects containing the particle type information and the numerical results themselves. This allows for more information to be stored in the object once the detector response to a certain particle type is well understood.

The second is that both a confidence level (sometimes referred to as a "consistency" or "significance level") and a likelihood value are provided for a given hypothesis. There is some discussion as to the relative merits of these (see for example [67]). Support for both approaches is desirable, since the likelihood method necessitates greater detector understanding (since the normalisation of it requires knowledge of the response to all known hypotheses), but provides more statistical power. Access to both of these is provided by the EmcPidInfo class. In addition, the value of the discriminating variable used is stored in this class.

Thirdly, it is envisaged that particle ID information will not come from just one algorithm. For example, electron identification may be achieved simply by looking at the ratio of the energy of the cluster to magnitude of the momentum of the track that caused that cluster. This quantity is close to one for electrons and in general different from one for all other charged particles. Additional discriminating power will come from looking at the shower shape of the resulting cluster. Analysts will want to compare these algorithms, and combine the results from both to form "composite 
algorithms." This has two consequences. One is that there is necessarily a "default" algorithm for a given particle type. In general this will be the algorithm giving the best discriminating power, but is also a somewhat arbitrary choice among those algorithms that are "best understood." The second is that comparisons between algorithms will be necessary. Thus the concept of asking for the confidence level that a given observation conforms to a given hypothesis using a particular algorithm is encoded into the EmcPidInfo class. In handling of the request for information when no algorithm is specified (i.e. the "default" algorithm), care is taken to ensure that the likelihoods so derived come from looking at the same (set of) discriminating variable(s).

\section{EmCandidateMaker}

The derivatives of this abstract class are responsible for constructing EmcCands which may conform to the particle hypothesis in question. An example is the use of the charged track and cluster associations described in section 3.4.2 to construct EmcCands which can then be passed to the various subclasses of EmcIdentifier (see below) which deal with charged particles, or the isolation of single clusters which are not charged for use in neutral hadron or $\pi^{0}$ identification. The purpose is to construct a list of EmcCands in the Event which will have calibration and particle ID information attached to them. How well these candidates conform to various hypotheses is the concern of EmcIdentifier subclasses. In general there will be more EmcIdentifier subclasses than those of EmcCandidateMaker.

\section{EmcIdentifier}

This abstract class has derivatives that are responsible for providing implementations which look at particular discriminating variables and provide information on the confidence level and likelihood for a given EmcCand under a given particle hypothesis. Usually this takes the form of comparing the properties of the EmcCand to a known probability density function and calculating the relevant mathematical quantities. (Classes have been provided in order to ensure that this calculation is consistent across different probability density functions.) EmcIdentifier subclasses contain strings which relate to the given algorithm they represent, as well as an object which relates to the particle type they are identifying. A specific subclass of EmcIdentifier, EmcCompositeIdentifier, employs a Template pattern [57] to allow the particular implementation to look at the results of several different identifiers and form a new conclusion based on this input. The most extreme example of this is a neural network.

The subclasses of EmcIdentifier that are asked to consider the EmcCands in a particular event are controlled by a Framework module which uses a factory pattern [57] to obtain the derivatives of EmcIdentifier that are required. This module itself is coded entirely in terms of the abstract interface. This allows for the extension of the number of particle types identified and the number of algorithms used to identify them. 


\subsubsection{Offline calibration}

The energy deposits observed in the calorimeter will represent only some fraction of the energy of the incident particle at its point of creation. For electromagnetic particles, this fraction is about $95 \%$, as a result of losses in the material before the calorimeter, notably the DIRC, leakage from the front and rear of the crystal and losses in the walls between crystals. It is necessary to apply a correction to the observed energy to "recover" these losses. In general this correction will be a function of energy, of polar angle, and of particle type. (Note that we may not wish to calibrate back to the incident energy, for example in the case of muons, but that some energy correction will need to be applied for all particle types is not in doubt.) The experiences of other experiments suggest that this correction, or calibration, will become a function of more and more variables as knowledge of the detector improves. An example for $B A B A R$ is that a correction for the azimuthal asymmetry of the DIRC may be applied to calorimeter clusters when sufficient data is available. Furthermore, an analyst running over a channel with a small branching ratio may wish to reprocess events with the best possible calibration, i.e. one that takes in all the known functionality, even if this were not available when the data were first taken. A final requirement is that the calibration system be such that the origin of the correction function be allowed to change such that the default is always the best most general purpose correction, but that if an analyst requires that for example the radiative bha-bha correction only be used, then that option is available to them.

The requirement then is for a system that supports multiple algorithms, is extendible in its functional dependence and is retrospectively applicable. The coefficients of the functions used for the energy correction must be stored in the object-oriented database used by $B_{A} B_{A R}$, Objectivity/DB[69]. This does not allow for storage of the functional form itself. The approach is to store the coefficients in the database, along with knowledge of the name of a transient class which contains the encoding of the functional form appropriate to those coefficients. In addition, the algorithm name used to generate the coefficients is stored and a map between the algorithm name and the coefficients is stored to provide the functionality of algorithm selection.

In addition, the usage of the database in $B A B A R$ dictates that the interaction with the database by client code be kept to a minimum. In practical terms, this means that objects in the database (persistent objects) are translated into objects in memory (transient objects) which are then used by client code. The advantages of this approach are threefold. Firstly, client code is not dependent on the details of the database. If the particular database implementation were to change this should affect only that code which is responsible for translating objects of one domain to the other. Secondly client code is not capable of manipulating persistent objects, which could result in data corruption. Thirdly, technical restrictions imposed by the database implementation (most notably in the area of what a persistent object may or may not contain) are confined to a specific set of data representation objects and do not permeate the whole code.

The classes used to implement this will now be described in more detail. 


\subsubsection{Retrieval}

\section{EmcAbsCalibrator}

This class contains the pure virtual functions which take an EmcCluster as input and return the energy or position of that cluster according to the calibration information contained within the class. The functional form of the calibration is described by subclasses. Verification of a new version of a particular calibrator is performed by means of a virtual function whose default implementation is to check that the new calibrator is of the same type, has the same length and the constants are within $20 \%$ of the previous set. More complicated verification procedures may be implemented by subclasses. It is instances of this class' derivatives that are given to EmcCands for calibration purposes. See section 3.4.3.

\section{EmcAbsCalibrator subclasses}

These implement particular functions for calibration, which assume knowledge of the coefficients that are given to them at construction time. They also override the default verification function if required. The creation of a new subclass would occur if either the function describing the calibration had changed or a different algorithm was being used to generate them, but is not necessary if only the coefficients have changed.

\section{EmcCalibratorP}

This class contains a persistent array of coefficients for a given calibration function, a persistent string which contains the name of the transient class (the EmcAbsCalibrator derivative) to which these constants pertain, and a third string to describe the algorithm used to derive the constants. Subclasses of EmcCalibratorP pertain to different algorithms and differ from the base class only in the content of the algorithm name string.

\section{EmcCalibProxy}

EmcCalibProxy provides the interface between clients of the EmcAbsCalibrator class and the database. The proxy mechanism is such that a given EmcAbsCalibrator subclass is held by the proxy and returned on demand until either the subclass relating to a different source of calibration data (referred to as an algorithm below) is requested, or the event time indicates that the current set of constants is out of date. Under these circumstances, the constants for a new derivative are retrieved from the database and a new transient EmcAbsCalibrator derivative constructed appropriate to these constants, using the information stored along with them. The specifics of this translation are referred to in more detail below. The general mechanism of proxies and its specific implementation in $B A B A R$ is discussed in [68].

\section{EmcCalibratorDictionary}

This class provides a persistent map between the algorithm names and the persistent calibrators (which are actually EmcCalibratorP derivatives) and thus removes the need for clients to know about the subclasses of EmcCalibratorP. The mechanics are that the persistent map of EmcCalibratorPs and algorithm names is loaded from the database and each EmcCalibratorP translated 
into a transient EmcAbs-Calibrator derivative. The persistent class contains a string which relates to a particular derivative of EmcAbsCalibrator, whilst the factory EmcCalibratorWarehouse is responsible for giving back the appropriate derivative based on this string. The dictionary then loads the constants it references in the EmcCalibratorP object into the transient EmcAbsCalibrator derivative. The appropriate derivative is passed back to the EmcCalibProxy based on the algorithm requested.

\subsubsection{Storage}

There are two aspects to the permanent storage. One is the ability to store the coefficients of the functional forms encoded in the EmcAbsCalibrator derivatives. The other is the ability to keep track of available calibration algorithms and of the default calibration. The classes EmcAlgDescription and EmcAlgListProxy are employed to perform the latter task, whilst these classes are used in conjunction with the EmcAbsCalibAlgorithm derivatives and EmcStoreCalibrator to perform the former task.

\section{EmcAlgDescription}

Persistent capable class containing a twenty character algorithm name and a flag indicating whether or not that algorithm is the default. These are placed in the database inside an EmcAlgBank object, while the transient equivalent used by client code is an EmcAlgList. These are nothing more than persistent (Bank) and transient (List) vectors.

\section{EmcAlgListProxy}

This class is responsible for the retrieval from the database of the relevant EmcAlgBank object pertaining to the event time. Its operation is very similar to the EmcCalibProxy in the sense that a persistent EmcAlgBank is translated into a transient EmcAlgList for client use, and this AlgList is replaced when the database indicates that a new version relating to the event time is available.

\section{EmcAbsCalibAlgorithm}

This class is responsible for generation of the constants to be stored in the database. This is envisaged as a long term, relatively complicated analysis or simply the reading in of the results of such an analysis (from a text file for example) for permanent storage. Each subclass of EmcAbsCalibAlgorithm is responsible for the generation of a particular derivative of EmcAbsCalibrator with the appropriate contents. This object is then passed to EmcStoreCalibrator which performs the mechanics of storage. Each subclass of EmcAbsCalibAlgorithm must register itself with EmcCalibAlgRegister which is responsible for keeping record of the available algorithms and an indication of the default.

\section{EmcStoreCalibrator}

Singleton (see [57]) class responsible for the storage of an EmcAbsCalibrator derivative from a given algorithm. The algorithm name is used to obtain a particular subclass of EmcCalibP, via a factory method object EmcCalibPCreator. The persistent class is loaded the constants which 
are verified using the verification function in the EmcAbsCalibrator derivative and the resulting persistent object is stored, updating the persistent EmcCalibDictionary.

\subsubsection{Offline calibration: summary}

The rather complicated design described above achieves several things at once. Firstly it separates the client code (coded in terms of EmcAlgLists and EmcAbsCalibrators) from the database implementation, as required. Secondly, in the form of the EmcAlgList/Bank object, and the Emc-CalibDictionary's map between the EmcAlgDescription objects and the EmcCalibratorP derivatives, the extensibility of the calibration scheme when new and more complicated algorithms become available is achieved. Thirdly, since these algorithms make no reference to time, they are by definition retrospectively applicable providing the information on which they are based is recorded in the database at the appropriate time. Fourthly, by providing differing implementations of the calibration functions while retaining the external code's dependence on an abstraction, new and more complicated calibration functions can be applied without needing to change external code.

\subsection{Chapter summary}

The basic concepts of object oriented programming and the motivation for using it in $B A B A R$ software have been described. The implementation of the last stage of simulation i.e. the crystal and front end electronics response to an energy deposit has been described along with the theory and implementation of the matched digital filter which is to be used in feature extraction for the calorimeter. The principle components of the reconstruction software for the calorimeter have been discussed, in particular with reference to the use of object oriented design techniques to allow for increased functionality once a better understanding of the detector has been reached. This software development has encompassed the entire chain of reconstruction from the front end electronics output to particle identification and calibration. 


\section{Neutral particles in the electromagnetic}

\section{calorimeter}

\subsection{Introduction}

One of the primary purposes of the electromagnetic calorimeter is the energy measurement and the identification of photons and $\pi^{0} \mathrm{~s}$. Many CP channels contain one or more $\pi^{0} \mathrm{~s}$ in their final states. Important examples are: $B \rightarrow \rho^{ \pm} \pi^{\mp}$ and $B \rightarrow a^{1 \pm} \pi^{\mp}$, measuring the angle $\alpha$ in the unitarity triangle, $B \rightarrow J / \psi K^{* 0}, B \rightarrow J / \psi K_{S}^{0}$ and $B \rightarrow D^{+} D^{-}$measuring $\beta$, and $B \rightarrow \pi^{0} \pi^{0}$, used to give an indication of the size of penguin contributions to the angle $\alpha$.

For $\pi^{0}$ reconstruction the most simple approach is the straightforward combination of individual photon clusters. This approach is suitable for low energy $\pi^{0} \mathrm{~s}$. However at higher energies, the two photons from the $\pi^{0}$ decay combine to form one cluster. The fraction of $\pi^{0} \mathrm{~s}$ "merging" to form one cluster as a function of energy is shown in figure 4-1. For these cases, two approaches are available. The first is to attempt to divide the cluster into isolated electromagnetic maxima, as described in section 3.4.1.2. This will be discussed in section 4.2.3. This approach has fundamental limitations, however, since at the highest energies, the daughter photons from the $\pi^{0}$ will approach each other such that the separation is less than two crystal widths. This makes the definition of such a pair as "isolated" electromagnetic clusters no longer possible. Table 4.1 shows the minimum separation of the daughter photons as a function of energy.

\begin{tabular}{|c|c|c|}
\hline $\begin{array}{c}\pi^{0} \text { energy } \\
(\mathrm{GeV})\end{array}$ & $\begin{array}{c}\text { Minimum photon separation }\left(90^{\circ}\right) \\
\text { crystal widths }\end{array}$ & $\begin{array}{c}\text { Minimum photon separation (fwd region) } \\
\text { crystal widths }\end{array}$ \\
\hline 0.5 & 10.7 & 22.0 \\
1.0 & 5.29 & 11.0 \\
2.0 & 2.63 & 5.6 \\
3.0 & 1.76 & 3.70 \\
4.0 & 1.32 & 2.79 \\
5.0 & 1.05 & 2.21 \\
\hline
\end{tabular}




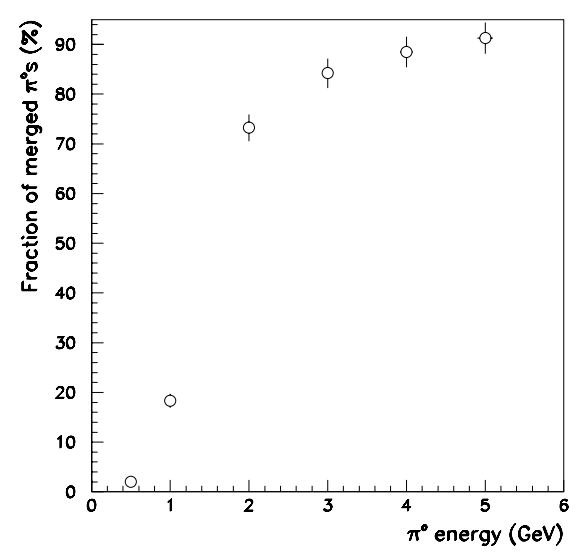

Figure 4-1. Fraction of $\pi^{0} s$ whose daughter photons are sufficiently close to form only one cluster as a function of energy.

Therefore a second method is adopted which identifies characteristics of showers arising from the two daughter photons which distinguish them from single photon clusters. This is discussed in section 4.3.

Photon reconstruction is essential for low energy $\pi^{0}$ reconstruction as well as being an important aspect in its own right. The average photon multiplicity in generic $B$ decays is 5.5 , and the overall $B$ reconstruction efficiency falls off dramatically as the minimum detectable photon energy increases (see figures 4-2 and 4-3)[70].

This task is made considerably more difficult in the presence of machine background, since a large number of soft photons are reconstructed that did not originate from the $\Upsilon(4 \mathrm{~S})$ decay. The number and energy spectrum of machine background photons is heavily dependent on the clustering parameters used, and these will be discussed, and the performance of the $B A B A R$ clustering algorithm compared to that used in the CLEO calorimeter.

The division of clusters to produce isolated electromagnetic maxima requires knowledge of the transverse profile of single photon clusters. The evaluation of this profile and the impact of the knowledge of it on $\pi^{0}$ reconstruction will be shown. The adaption of the $\pi^{0}$ identification technique to photons will be discussed and the possible use of the transverse profile as an aid to identification.

An example of these techniques, applied to the $\mathrm{CP}$ channel $B \rightarrow J / \psi K_{S}^{0}$ will be shown in chapter 5 . 


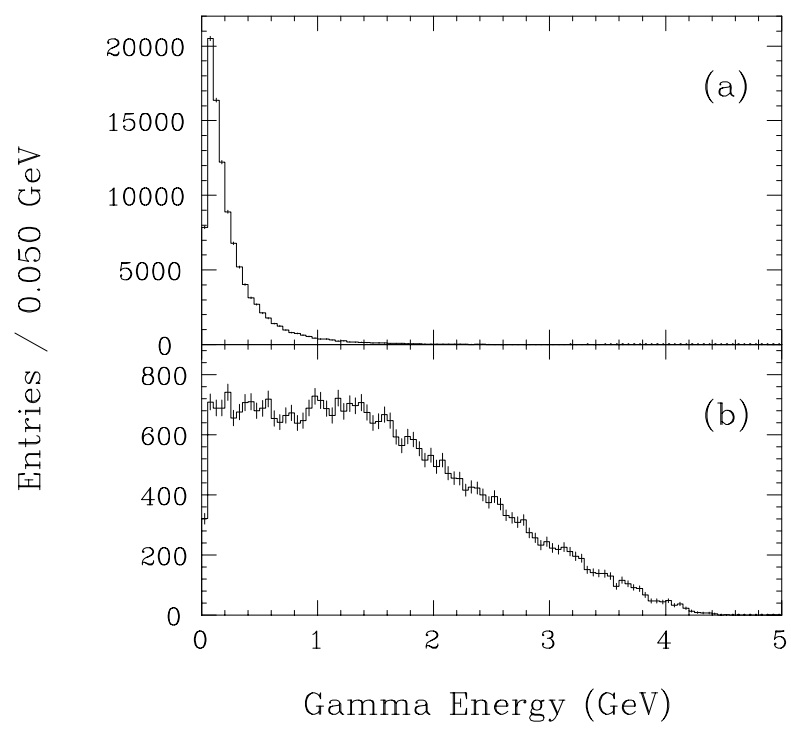

Figure 4-2. Photon energy spectrum for generic $B$ decays at BABAR (upper plot) and from the most energetic decay, $B \rightarrow \pi^{0} \pi^{0}$ (lower plot). The sturcture in the lower plot is purely statistical.

\subsection{Reconstruction parameters}

\subsubsection{Clustering parameters}

The clustering algorithm has been described in chapter 3. The three thresholds which impact on the algorithm's performance, both in terms of resolution and combinatorics from machine background, are the digi inclusion threshold, the seed threshold and the cluster energy threshold: these terms are now briefly recalled.

If a crystal is over a seed threshold it is considered as a starting point for a cluster. The neighbours (including corners) of this crystal are then examined. If they are above an energy threshold, a digi threshold, they are included in the cluster. If any of these is above the seed threshold, then its neighbours are considered. This algorithm is applied recursively until no more crystals are above the seed threshold. The resulting assembly, if it passes a third energy cut, hereafter a cluster threshold, is added to the event cluster list.

In addition, there is a sparsification cut in the hardware which allows only certain crystals to be passed through the digital filtering algorithm and read out. This is constrained by available bandwidth and the processing power required for digital filtering, not resolution or combinatoric background, its variation will not generally be considered here. The current algorithm used for study allows for crystals to undergo this process if they have an analogue energy of $10 \mathrm{MeV}$, or are neighbours of such a crystal. 


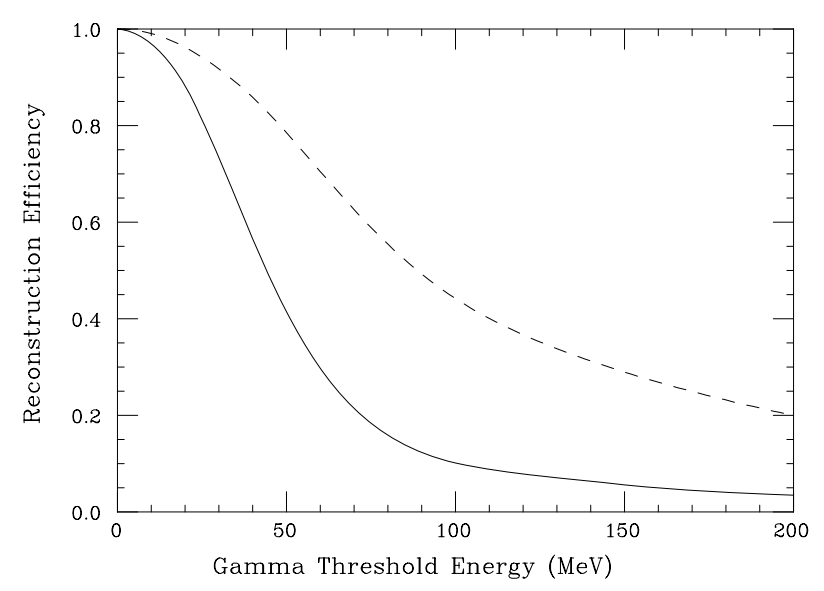

Figure 4-3. Efficiency for $\mathrm{B}^{0}$ reconstruction (solid line) and $\pi^{0}$ reconstruction (dashed line)

versus minimum detectable photon energy.

The spectrum of energy deposits in individual crystals from machine background can be found in figure 3-2. An indication of the size of the problem is given by the fact that at ten times nominal machine background, approximately $3 \mathrm{GeV}$ per physics event is deposited in the calorimeter in a time window equivalent to the trigger jitter during which feature extraction can occur. Latest data from PEP-II [71] indicate that the "nominal" levels of background are too low by a factor 5-7. Studies at ten times nominal machine background will therefore be included.

\subsubsection{Digi and seed thresholds}

The number of clusters observed in an event at ten times nominal background can be very large (see figure 4-4). The clustering algorithm was run with a standard $20 \mathrm{MeV}$ cluster cut (the typical minimum energy of a cluster as defined in [60]), a varying seed cut and a varying digi cut. For comparison, a $5 \mathrm{MeV}$ sparsification cut is shown. The number of clusters observed from machine background is largely independent of the digi inclusion cut. This observation will allow this cut to be optimised with respect to resolution only. The seed crystal cut shows a slightly greater impact on the number of clusters observed. This is especially when it approaches the same value as the digi inclusion cut. At such values, the background rate is such that clusters of several hundred crystals can form, and the clustering algorithm essentially fails. It is for this reason that the number of clusters falls at low seed energy cut values. In order to preserve efficiency, however, this cut should be as low as possible, i.e. the value above which it has no impact on the observed number of clusters. This is estimated to be $3 \mathrm{MeV}$ for the current understanding of ten times nominal background. 


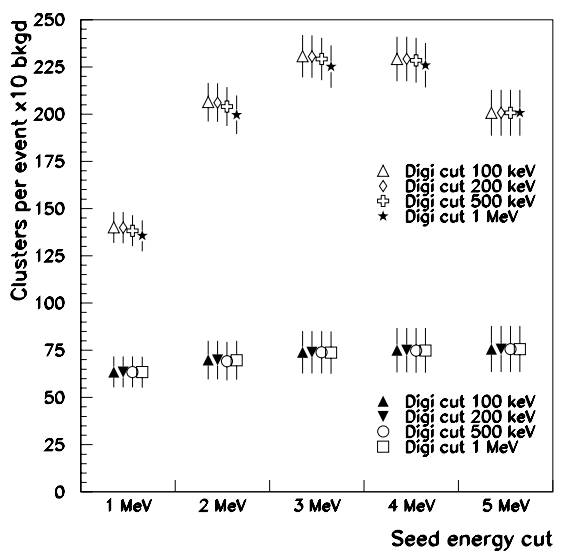

Figure 4-4. Number of observed clusters above $20 \mathrm{MeV}$ for ten times nominal machine background, for sparsification cuts of $5 \mathrm{MeV}$ (top) and $10 \mathrm{MeV}$ (bottom), and various seed crystal and digi energy cuts. The points have been separated horizontally for clarity.
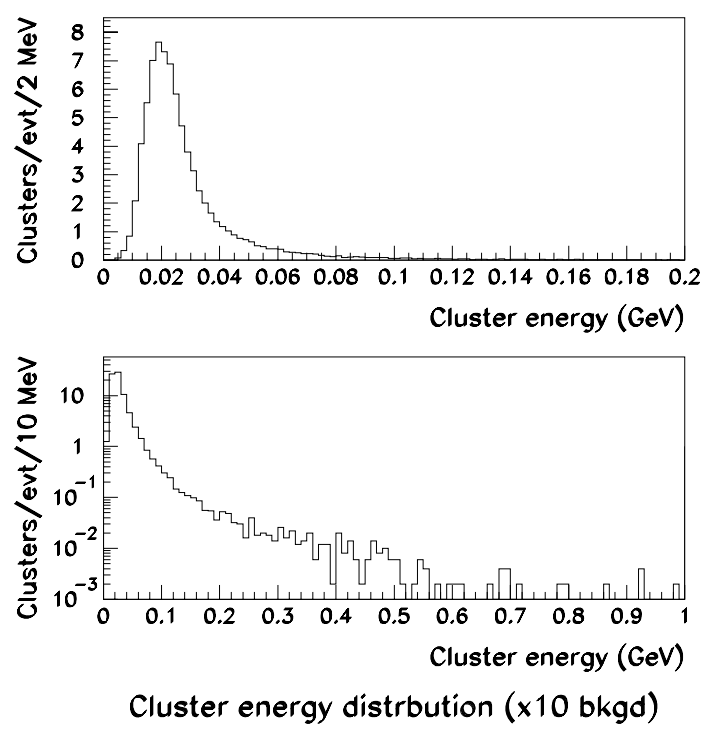

Figure 4-5. Energy spectrum of clusters from ten times nominal background, using standard clustering parameters. 


\subsubsection{Seed and cluster thresholds}

The distribution of energy of background clusters above $5 \mathrm{MeV}$ is shown in figures 4-5. Using various seed energy cuts, the number of clusters per event as a function of cluster cut was determined and the results are shown in figure 4-6. For reference, a set of points using a seed cut of 3 $\mathrm{MeV}$ but without the digital filtering used in the feature extraction are also included.

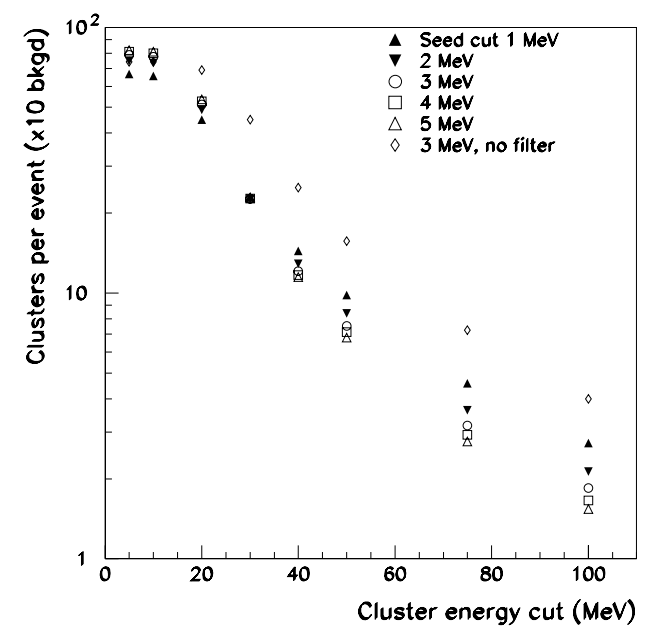

Figure 4-6. Number of observed clusters at times ten nominal background.

The number of observed clusters at this level of background is very large. The seed threshold has some effect of reducing this number, but no significant reduction is observed above $3 \mathrm{MeV}$. For this reason and the limit imposed by its effect in conjunction with the digi cut, the value of $3 \mathrm{MeV}$ is adopted. While the cluster energy cut should be as high as possible to reduce this number, this has a severe impact on the overall $B$ reconstruction efficiency, as noted above. The impact will be analysis dependent, and is evaluated for the particular case of $B \rightarrow J / \psi K_{S}^{0}$ in chapter 5 .

\subsubsection{The CLEO clustering algorithm}

The CLEO clustering algorithm as described in [60] is somewhat different to the one presently implemented. Firstly, a crystal must be above a certain threshold $(10 \mathrm{MeV})$ to seed a cluster, and it must be a maximum amongst its neighbour crystals up to two deep. Passing these two criteria, 
a cluster is then the collection of this maximum, its neighbours, and their neighbours. The energy is determined by taking the highest $\mathrm{N}$ crystals when $\mathrm{N}$ is 4 for energies less than $25 \mathrm{MeV}, 17$ for more than $4 \mathrm{GeV}$, and varies as the log of the "raw" energy of the collection in between. Shared crystals are divided amongst the clusters according to the energy of their maxima.

A comparison of the two algorithms' resolution can be seen in figure 4-7. The fitted function is a parameterisation of the resolution curve developed at Novosibirsk:

$$
f(E)=A \exp \left[-\frac{1}{2}\left(\frac{\log \left(1+\tau(E-\mu) \frac{\sinh (\tau)}{\sigma \tau \sqrt{\log 4}}\right)}{\tau}\right)+\tau^{2}\right] .
$$

The parameter $\tau$ describes the tail on either side of the peak, being positive if the peak lies to the high energy side and negative otherwise. The parameter $\sigma$ is the full width at half maximum divided by 2.36. This is the quoted resolution. An example of such a fit can be seen in figure 3-4. In the limit as $\tau \rightarrow 0$, the function tends to a Gaussian. This function and three others used to describe energy deposits in the calorimeter are discussed in [72]. The top plot shows the resolution with no background and nominal background, the lower 10 times nominal. A similar plot for efficiency can be found in figure 4-8. The top plot refers to no background, the lower to nominal and ten times nominal. Efficiency is defined as the fraction of observed clusters that have energies within 5 sigma of the nominal energy. Machine background photons are excluded, except in the case of $40 \mathrm{MeV}$ photons, where the width is such that a very large number the background photons would be included in this definition.

The resolution distribution for $40 \mathrm{MeV}$ photons is highly non-Gaussian, and their observation is extremely difficult in high background conditions. A study of the timing resolution on the individual crystals suggests that this may improve resolution [73], but this has not been investigated here. The mass resolution for $500 \mathrm{MeV} \pi^{0}$ is shown in figure 4-9. This energy is chosen since approximately $99 \%$ of $\pi^{0}$ at this energy deposit isolated photons in the calorimeter (see section 4.1), and the mass resolution is not, therefore, a function of the division of clusters into isolated electromagnetic maxima. The mass peak at ten times nominal background is dominated by combinatoric background and a straightforward combination of all observed clusters cannot be used at this background level. Analysis specific reconstruction techniques will have to be employed if the number of clusters cannot be reduced at this level of background.

There is no significant advantage to either algorithm in the performance issues discussed here. The approach of allowing contiguous regions of arbitrary size allows for techniques such as the isolation of electromagnetic maxima (both to reconstruct $\pi^{0} \mathrm{~s}$ and also to reject machine background) and the use of complex cluster shape parameters in particle identification. As this approach does not present a significant performance disadvantage to the more conventional technique, it is adopted here. 

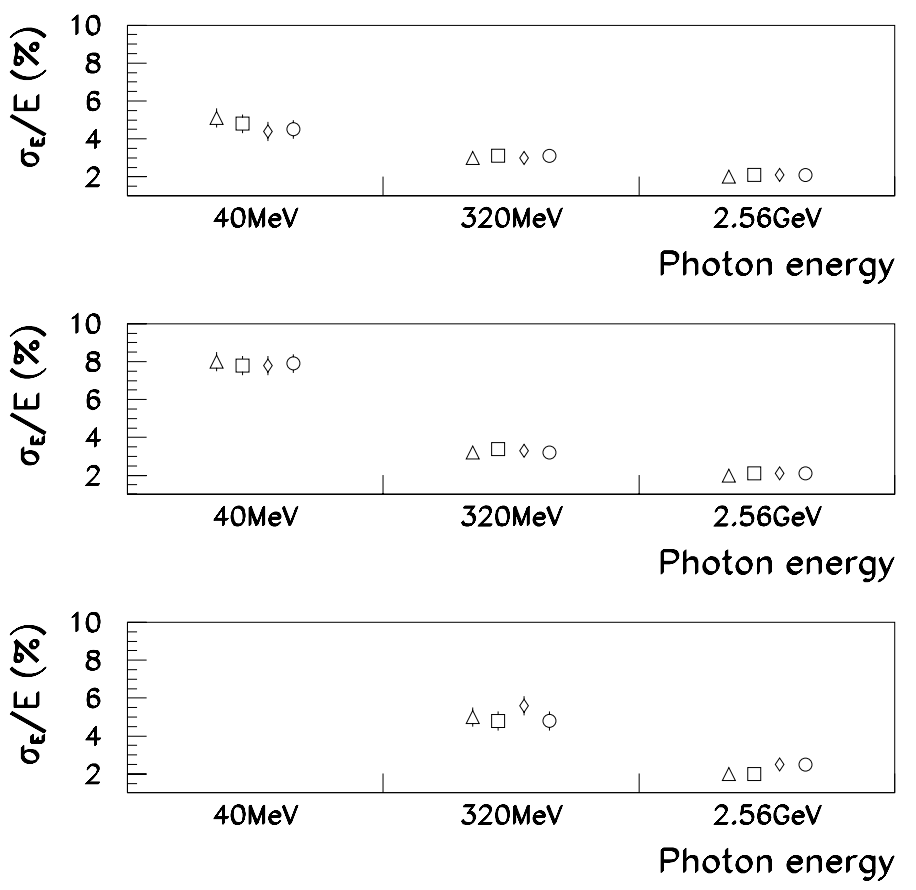

Figure 4-7. Comparison of the CLEO clustering algorithm's resolution to the projected performance for the BABAR algorithm. Each set of four points corresponds to (left to right): CLEO algorithm taking all crystals (denoted $\triangle$ ), CLEO algorithm with the number of crystals limited according to the algorithm described in the text $(\square)$, BaBar algorithm taking all crystals $(\diamond)$, and BaBar algorithm with a limited number of crystals $(O)$. The top plot assumes no machine background, the middle nominal levels and the lower plot ten times nominal levels. $40 \mathrm{MeV}$ photons have too poor a resolution at ten times nominal background to be described well by the function given in the text and are omitted from the above plot. 

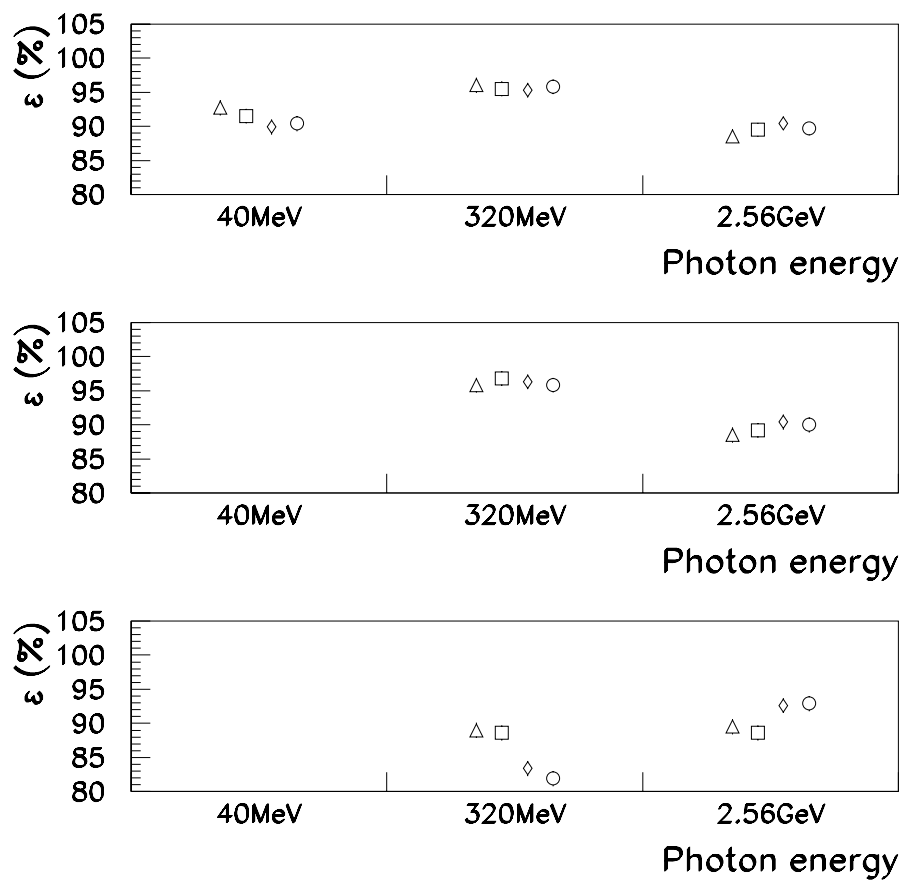

Figure 4-8. Comparison of the CLEO clustering algorithm's efficiency to the projected performance for the BABAR algorithm. Each set of four points corresponds to (left to right): CLEO algorithm taking all crystals $(\triangle), C L E O$ algorithm with a limited number of crystals ( $\square)$, BaBar algorithm taking all crystals $(\diamond)$, and BaBar algorithm with a limited number of crystals $(O)$. The top plot assumes no machine background, the middle nominal levels and the lower plot ten times nominal levels. At nominal background, the $40 \mathrm{MeV}$ photon resolution is such that too many machine background photons would be included in the efficiency calculation as described. The resolution (and therefore efficiency) cannot be determined at ten times nominal background for $40 \mathrm{MeV}$ photons. 


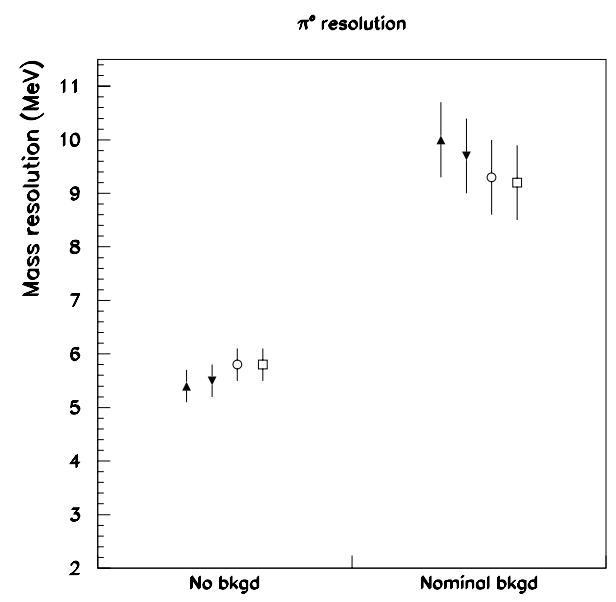

Figure 4-9. Comparison of the CLEO and BABAR clustering algorithms' mass resolution for $500 \mathrm{MeV} \pi^{0} s$.

\subsubsection{Cluster splitting parameters}

As noted in section 4.1, a cluster, or contiguous region of crystals, may contain more than one electromagnetic maximum. The division of the cluster into isolated maxima, or bumps, is crucial. Firstly it provides an approach for the reconstruction of medium and high energy $\pi \mathrm{s}$, above about $1.5 \mathrm{GeV}$. Secondly it provides a mechanism to remove extraneous machine background photons from an otherwise bona fide energy deposit. Thirdly it can provide additional information to particle identification, such as the case of an electron which has a bremsstrahlung photon close to the impact point in the calorimeter. The software described in chapter 3 allows for the original clusters to be preserved even after bump creation, which allows for the study of hadronic clusters which may fake electromagnetic maxima, or as an alternative way of reconstructing "merged" $\pi$ s (see section 4.3).

The location of electromagnetic maxima is performed by comparing the energy of a given crystal with its neighbours. The ratio between the energy of the potential maximum and the highest of its neighbours is usually large for electromagnetic showers (see figure 4-10). Similarly the ratio of the largest energy crystal to the average of those of its neighbours is also large (see figure 4-11).

Using these criteria, the determination of electromagnetic maxima is then optimised such that the number seen in a photon sample is generally close to one, whilst trying to minimise the number of maxima seen in hadronic samples. In figure 4-12, the number of observed maxima is shown against the ratio required for those maxima to exist, both comparing a crystal with the neighbouring crystal of highest energy, and to the average energy of its neighbours.

The importance of minimising the creation of "fake" maxima becomes clear in reconstruction of medium energy $\pi^{0} \mathrm{~s}$. If the criteria for maxima location is too low, fake maxima create low mass photon pairs giving a large combinatoric background to the $\pi^{0}$ sample. Using a simulated single 
particle $2 \mathrm{GeV} \pi^{0}$ sample, the maxima location criteria is optimised such that the combinatoric background from fake bumps is flat. The ratio of the highest energy crystal in the maximum to the highest of its neighbours in this case is in the region of 2.0, and the ratio with respect to the average energy of its neighbours approximately 7.0. As can be seen from figure 4-12, this corresponds to on average approximately 1 maxima being created for each photon in the event in both cases. The combinatoric background from both methods is then approximately equal. The comparison to the average energy of neighbouring crystals is slightly more efficient and is adopted.

The division of the cluster into bumps associated with each cluster requires knowledge of the electromagnetic shower shape. The fraction of a crystal's energy associated to a particular maxima, the weight, should be such that the lateral profile of the resulting bump is as close as possible to the lateral profile of a single photon. This has been determined by examining the energy assigned to crystals whose lateral separation from the centroid is outside a given distance, using 500 photon showers each of energies 40,80,160,320, 1280 and $2560 \mathrm{MeV}$. Typical results are shown in figure 4-13. The functional form is a two component exponential as discussed in [74] with extra terms linear and quadratic in radial distance to account for geometry. Some larger geometrical effects are seen for very high energy showers. The evolution of the fit parameters in terms of energy was evaluated in terms of polynomials of order up to 3 to allow for the profile of a cluster of arbitrary energy to be determined.

Ratio of highest energy crystal to highest neighbour
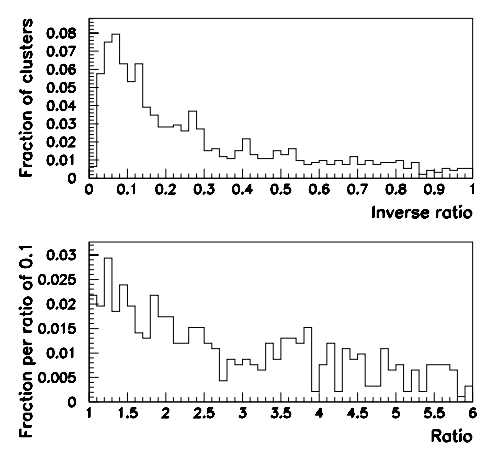

Ratio of highest energy crystal to highest neighbour

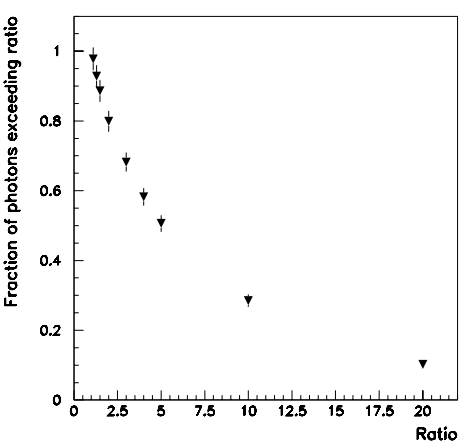

Figure 4-10. The comparison of the highest energy crystal in a shower to the highest of its neighbours for medium energy ( $320 \mathrm{MeV}$ ) photons. (Left-bottom) The ratio of the highest energy crystal to the highest of its neighbours. (Top) The inverse of this quantity. (Right) The number of photon clusters whose highest energy crystal/highest energy neighbour crystal ratio exceeds a given value. 


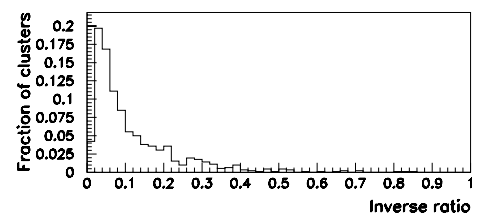

Ratio of highest energy crystal to highest neighbour
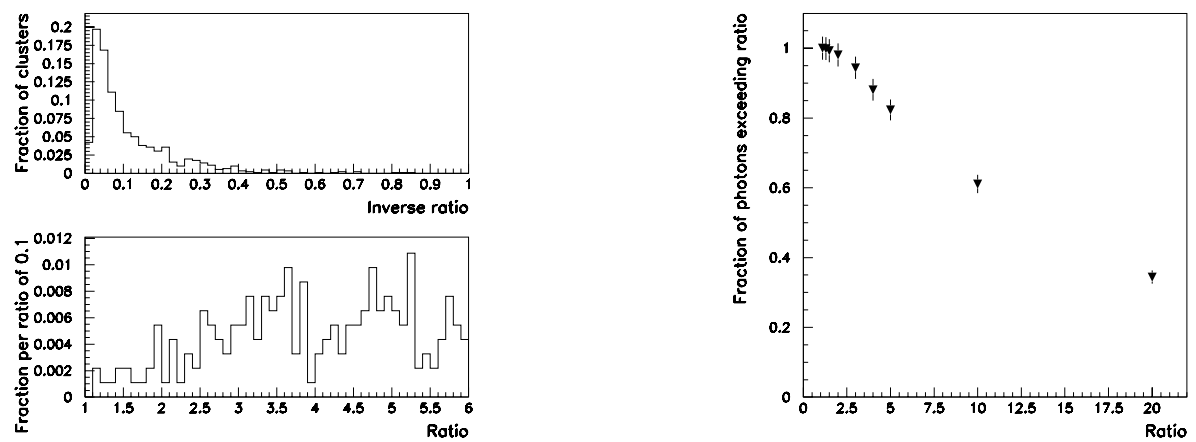

Figure 4-11. The comparison of the highest energy crystal in a shower to the average of its neighbours for medium energy ( $320 \mathrm{MeV}$ ) photons. (Left-bottom) The ratio of the highest energy crystal to the average of its neighbours. (Top) The inverse of this quantity. (Right) The number of photon clusters whose highest energy crystal/average energy of neighbours ratio exceeds a given value.

\section{3 $\pi^{0}$ and photon identification}

\subsubsection{Discriminating variables}

A technique for $\pi^{0}$ identification was developed for the Crystal Ball calorimeter [75] and is adapted here. The second moment of a cluster is defined as:

$$
S=\frac{\Sigma_{i} E_{i} \boldsymbol{\Theta}_{\mathbf{i}}^{2}}{\Sigma_{i} E_{i}}, \quad, \boldsymbol{\Theta}_{\mathbf{i}}=\left(\begin{array}{c}
\theta_{i} \\
\phi_{i}
\end{array}\right) .
$$

The definition in terms of polar and azimuthal angle differs from [75] and is preferred as the variation of this quantity with polar angle in the $B A B A R$ calorimeter is less marked than the Crystal Ball definition, and is more suited to a non-spherical geometry. The treatment in this process of each crystal as an individual energy deposit allows for the "mass" of a given energy deposit to be derived. If $S_{\gamma}$ is the second moment of a photon of a given energy $E$, and $S$ the second moment of a $\pi^{0}$ cluster of the same energy, then

$$
m_{\pi^{0}}^{2} \simeq\left(S-S_{\gamma}\right) E^{2}
$$

the approximation becoming more accurate for a spherical, finely segmented calorimeter. The distribution of this quantity for a $3 \mathrm{GeV} \pi^{0}$ sample is shown in figure 4-14. The second moment 

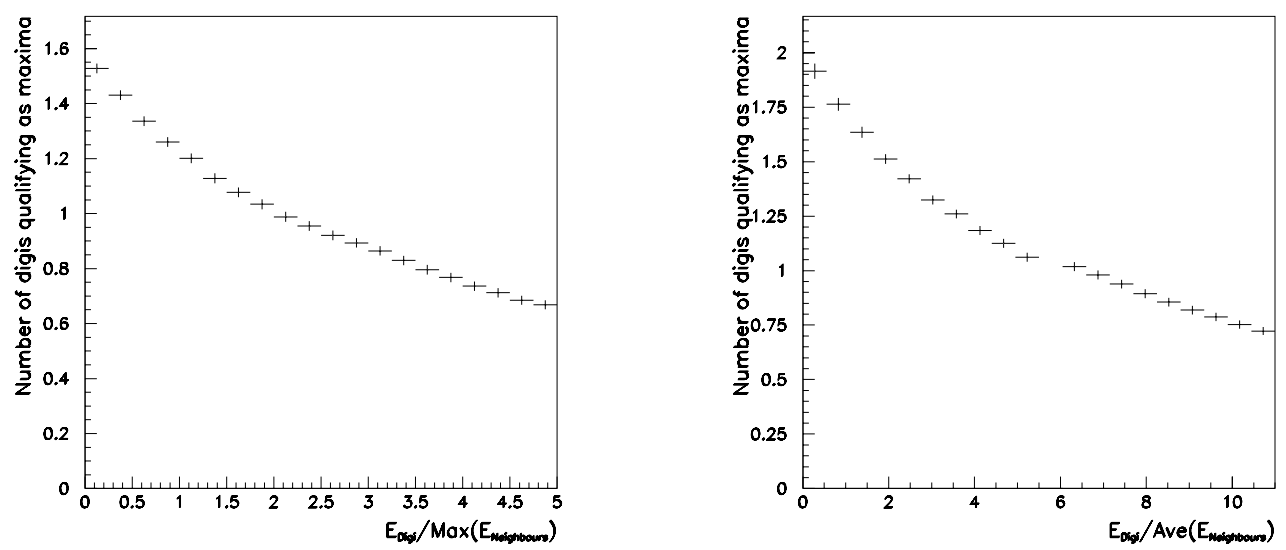

Figure 4-12. Number of maxima observed in one cluster for $320 \mathrm{MeV}$ photons as a function of the ratio between the candidate maximum and (left) the highest energy neighbouring crystal and (right) the average energy of neighbouring crystals.

provides a clear distinction between those $\pi^{0} \mathrm{~s}$ whose daughter photons form two distinct clusters and those which form one cluster. A distinct distribution for both types of occurrence allows the second moment to be adopted for both "merged" $\pi^{0}$ identification and for photon identification. The probability density function for the second moment is fitted empirically to

$$
f(x)=p_{1} \exp \left(-0.5\left(\left(x-p_{2}\right) / p_{3}\right)^{2}\right)+p_{5}\left(x-p_{4}\right)^{2} \exp \left(\left(x-p_{4}\right) / p_{6}\right) .
$$

The result of this fit for $2 \mathrm{GeV} \pi^{0} \mathrm{~s}$ is shown in figure 4-16. For $\pi^{0} \mathrm{~s}$, the peak position is found to be energy dependent, as expected. This is not the case where the showers originate from single photons.

The evolution of the fit parameters with energy of the incident $\pi^{0}$ was determined by evaluating the second moment distributions for $1-5 \mathrm{GeV} \pi^{0} \mathrm{~s}$ at intervals of $1 \mathrm{GeV}$. The evolution of each parameter was then described by either a straight line or a quadratic fit. This allows for the determination of the appropriate probability density function (pdf) for a $\pi^{0}$ of arbitrary energy.

As noted above, the second moment distribution is also suitable for identifying photons. The distribution was evaluated for photons of energy between $80 \mathrm{MeV}$ and $5.12 \mathrm{GeV}$. Below 80 $\mathrm{MeV}$, photon clusters are typically only one or two crystals, and this leads to the ill-definition of the second moment.

The determination of efficiency and purity for these identification techniques is done by calculating the confidence level for the given observation to be consistent with the appropriate hypothesis. The 

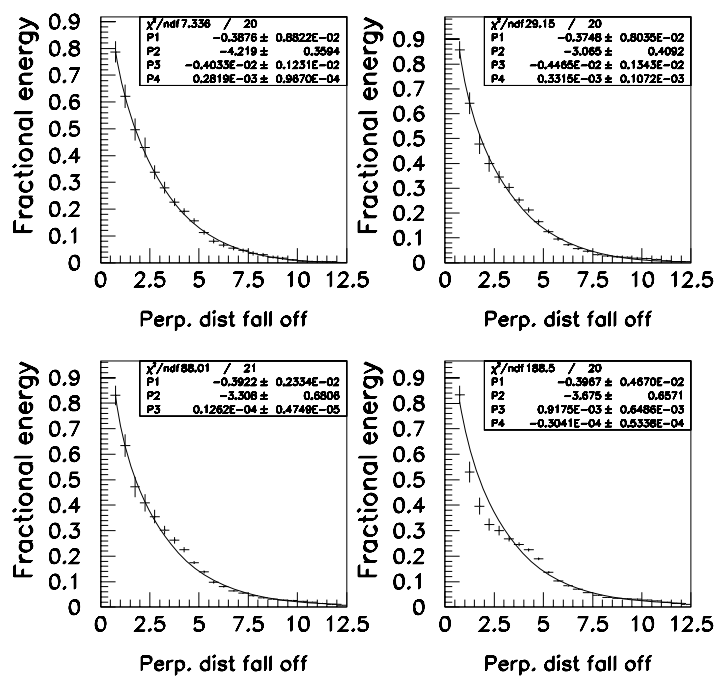

Figure 4-13. Lateral profile of photon clusters. The horizontal axis shows the lateral distance from the centroid in $\mathrm{cm}$ at the nominal shower maximum, and the vertical axis the fraction of the cluster energy contained outside that distance. Clockwise from top left are the profiles for 160 $\mathrm{MeV}, 320 \mathrm{MeV}, 2.56 \mathrm{GeV}$ and $1.28 \mathrm{GeV}$ photons.

calculation is performed by integrating the pdf as described in [78] and discussed further in [79]. A full likelihood determination is not performed here.

Figures 4-17 and 4-18 show the efficiency for photon and $\pi^{0}$ detection as a function of the probability for faking the other particle. Very high energy $\pi^{0}(\sim 4 \mathrm{GeV}$ and above) are somewhat difficult to distinguish from photons. However, the soft energy spectrum of photons in generic $B$ decays allows for a relatively clean sample of $\pi^{0} \mathrm{~s}$ in this region.

The lateral profile of electromagnetic clusters, discussed in section 4.2.3 in reference to cluster division can in principle be used for identification, since a true photon shower should have a profile consistent with the expected double exponential fall off. Consequently a given crystal's energy should be predictable as a function of its distance from the centre of a cluster, and the total deviation from the expected energy for all crystals in a cluster should provide for discrimination between particle types. However, distinct distributions are not observed for different particle types 

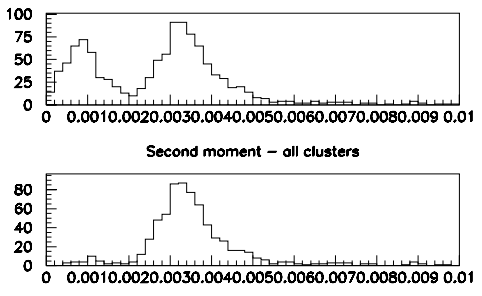

Second moment - two photon clusters

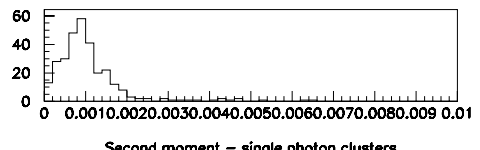

ment - single photon clusters

Figure 4-14. Distribution of the second moment of clusters in a $3 \mathrm{GeV} \pi^{0}$ sample. A clear separation of those $\pi^{0} s$ forming one cluster from those creating two (single photon) clusters is demonstrated by using the Monte-Carlo truth information.

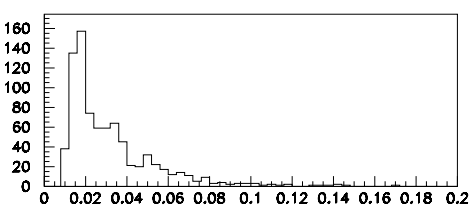

Squared deviation $1.28 \mathrm{GeV}$

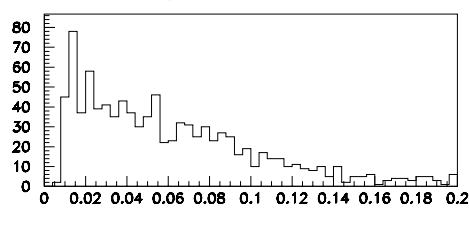

Squared deviation $\pi^{\circ} 4 \mathrm{GeV}$

Figure 4-15. Average squared deviation (in $\mathrm{GeV}^{2}$ ) of the observed energy of crystals in a cluster from the electromagnetic prediction for $1.28 \mathrm{GeV}$ photons (top) and $4 \mathrm{GeV} \pi^{0} s$ (bottom). Different, but not well separated, distributions are observed. 


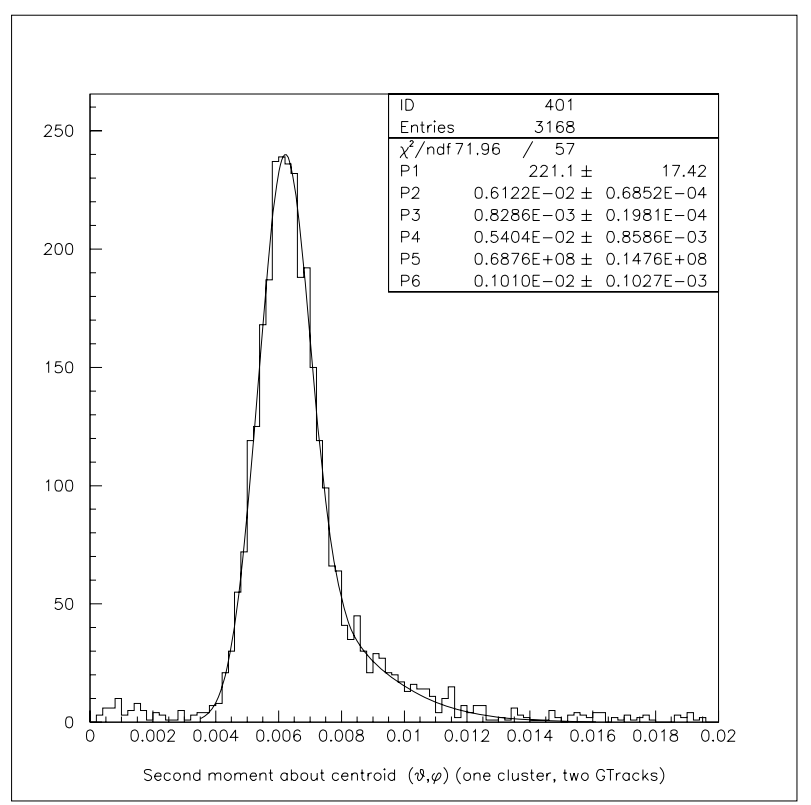

Figure 4-16. Second moment distributions for $2 \mathrm{GeV} \pi^{0} s$. The fit function is described in the text. The peak position varies as $\sim 1 / E^{2}$.

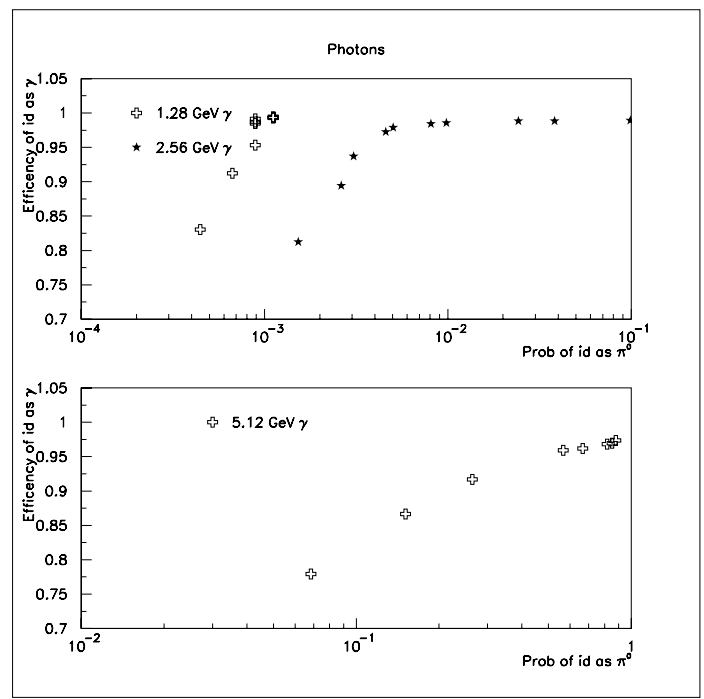

Figure 4-17. Efficiency of identification against probability of mis-identification for photons at various confidence levels for the observed energy deposit to be compatible with the photon hypothesis. 
(see figure 4-15), and as such this variable could only be useful as part of a multi-variant or neural network identification process. This is allowed for in the calorimeter particle identification framework described in chapter 3, but is not investigated further here.

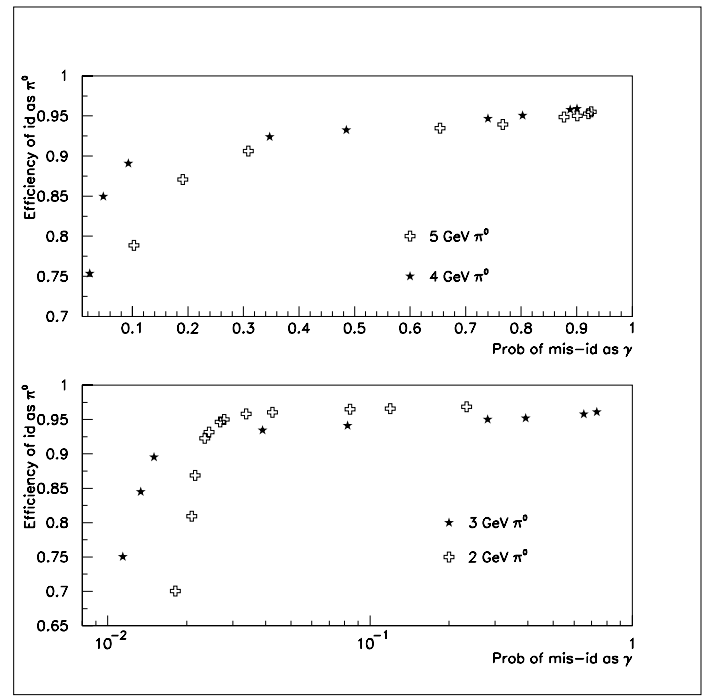

Figure 4-18. Efficiency of identification against probability of mis-identification for $\pi^{0} s$ at various confidence levels for the observed energy deposit to be compatible with the $\pi^{0}$ hypothesis.

\subsection{Chapter Summary}

The reconstruction of photons and $\pi^{0} \mathrm{~s}$ in the $B A B A R$ calorimeter has been described. The parameters used in the clustering algorithm have been evaluated with respect to their performance in background conditions. The digi inclusion cut has almost no impact on the number of observed clusters originating from machine background, and so is preserved at a nominal $0.5 \mathrm{MeV}$, relating to $3 \sigma$ of electronics noise. The seed cut for clustering does have a significant impact, and if it is too low (around the level of the digi inclusion cut) causes the clustering algorithm to fail. A level of around $3 \mathrm{MeV}$ is preferred. The cluster energy cut provides an efficient mechanism of reducing machine background but has a severe impact on $B$ reconstruction efficiency, and is therefore evaluated with respect to specific analyses (see for example section 5.5.3). The BABAR clustering algorithm has been evaluated against the CLEO algorithm, and is found not to present significant differences in resolution or efficiency.

Two methods of $\pi^{0}$ reconstruction have been evaluated: reconstruction by combination of isolated photons and two photon cluster division, and by showershape analysis on two photon clusters. The optimisation of the cluster division with respect to the typical lateral profile of photons in the $B A B A R$ calorimeter has been described and its effect on $\pi^{0}$ reconstruction shown. The use of the second 
moment of a cluster to identify merged $\pi^{0} \mathrm{~s}$ has been evaluated and been shown to provide good discrimination between photon and $\pi^{0}$ clusters, as well as a means of identifying photons. A study using the lateral profile of photons as a form of identification indicated insufficient discriminating power to be used as a single discriminating variable. 


\section{Study of the channel}

$$
B^{0} \rightarrow J / \psi K_{S}^{0} \rightarrow l^{+} l^{-} \pi^{0} \pi^{0}
$$

\subsection{Introduction}

The decay $b \rightarrow c \bar{c} s$ represents a theoretically clean environment for measuring the angle $\beta$ of the unitarity triangle. The weak ( $C P$ violating) phase for this transition from the tree diagram is the same as that from the dominant penguin contribution (see figure 5-1). Other penguin contributions

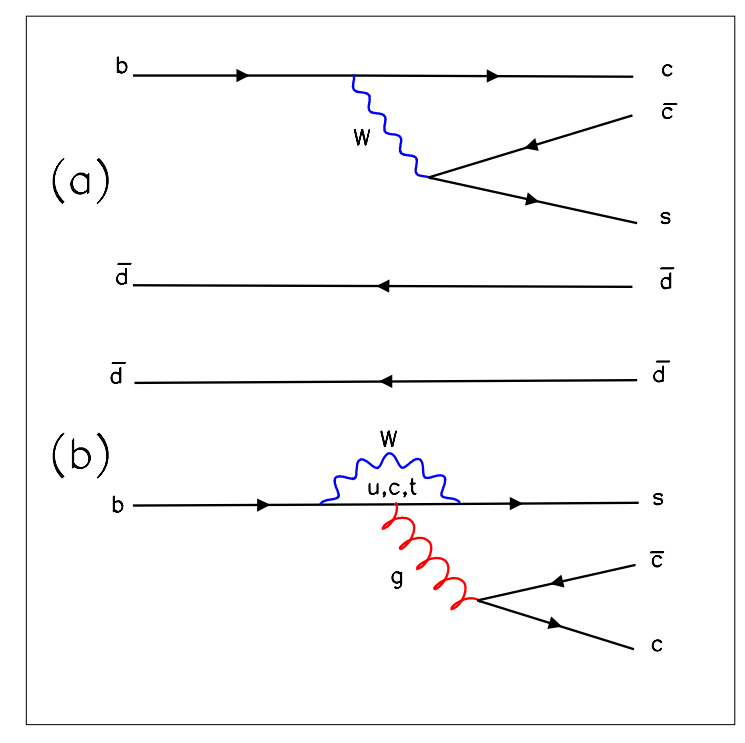

Figure 5-1. Tree (a) and penguin (b) diagrams for $\mathrm{B}^{0} \rightarrow J / \psi K_{S}^{0}$.

are doubly Cabbibo suppressed by a factor $\sim \sin ^{2} \theta_{c}$. Therefore to a good approximation the ratio 
$\lambda_{f_{C p}}$ defined in equation 1.52 ,

$$
\left|\lambda_{f_{C P}}\right|=\frac{q}{p} \frac{\bar{A}}{A}=1
$$

Under these circumstances, the time dependent asymmetry defined in equation 1.55 is given by

$$
a_{f_{C P}}(t)=-\operatorname{Im}\left(\lambda_{\mathrm{f}_{\mathrm{CP}}}\right) \sin (\Delta \mathrm{Mt}) .
$$

For $\mathrm{B}^{0} \rightarrow J / \psi K_{S}^{0}, \lambda_{f_{C P}}$ is given by

$$
\lambda_{f_{C P}}=-\left(\frac{V_{t b}^{*} V_{t d}}{V_{t b} V_{t d}^{*}}\right)\left(\frac{V_{c s}^{*} V_{c b}}{V_{c s} V_{c b}^{*}}\right)\left(\frac{V_{c d}^{*} V_{c s}}{V_{c d} V_{c s}^{*}}\right)
$$

the terms arising from $B$ mixing, the decay itself and $K$ mixing respectively. Recalling the definition of $\beta$,

$$
\beta=\operatorname{Arg}\left(-\frac{V_{c b}^{*} V_{c d}}{V_{t d} V_{t b}^{*}}\right),
$$

it immediately follows that

$$
\operatorname{Im}\left(\lambda_{\mathrm{f}_{\mathrm{CP}}}\right)=\sin 2 \beta
$$

The $\mathrm{B}^{0} \rightarrow J / \psi K_{S}^{0}$ decay in the above equation is experimentally the simplest method of extracting $\sin 2 \beta$. The $J / \psi$ is reconstructed in the lepton channel, which has a branching ratio of $0.1203 \pm$ 0.0019 [9] which provides for a very clean signal. The $K_{S}^{0}$ is looked for in the decay mode $K_{S}^{0} \rightarrow$ $\pi^{0} \pi^{0}$, which has a branching ratio of $0.3139 \pm 0.0028$, which is to be compared with the dominant mode $\left(\pi^{+} \pi^{-}\right)$of $0.6861 \pm 0.0028$ [9]. This mode gives an important test of the performance of the calorimeter software discussed in chapters 3 and 4 , as well as making a contribution to the experimental sensitivity to $\sin 2 \beta$. The latest data on the branching ratio of $B \rightarrow J / \psi K^{0}[80]$ indicate a branching fraction for this mode as $4.3 \pm 0.1 \times 10^{-4}$.

\subsection{Reconstructing the $J / \psi$}

\subsubsection{Tracking requirements}

The $B A B A R$ tracking software is described in [76] and a recent assessment of its performance can be found in [31]. Tracks are found independently in the drift chamber (DCH) and silicon vertex tracker (SVT). SVT tracks are required to have hits in four of the five layers. DCH tracks are required to have hits in all ten superlayers which are subsequently fitted to a helical track, or have hits in three layers, which are fitted to a helix, which is then projected into the rest of the chamber, adding track segments until the track can no longer be considered of sufficient quality. The former algorithm is more efficient for high $p_{\mathrm{t}}$ tracks, the latter for lower $p_{\mathrm{t}}$ tracks. SVT and DCH tracks are then projected to a radius of $20 \mathrm{~cm}$, corresponding to the location of the support tube, and matched where appropriate. 


\subsection{2 $J / \psi$ reconstruction}

The $J / \psi$ is reconstructed in the channel $J / \psi \rightarrow l^{+} l^{-}$. All pairs of oppositely charged good tracks
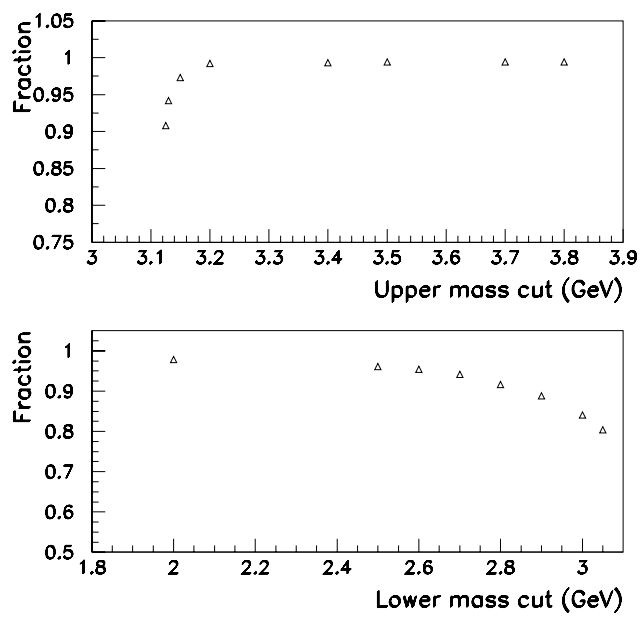

Figure 5-2. $\quad J / \psi$ efficiency as a function of upper and lower mass cut. The effects of acceptance are excluded. In each case the other mass cut is held constant at the nominal value described in the text.

are considered. The mass resolution for the $J / \psi$ (see figure 5-3) is expected to be $12 \mathrm{MeV}$ when the $J / \psi$ is reconstructed in both lepton channels. The resolution is expected to improve once the bremsstrahlung photons from the electrons are included, but this study is not performed here. A wide cut, $3.0<m_{l^{+} l^{-}}<3.2 \mathrm{GeV}$ is therefore placed on the mass to include the lower mass candidates caused by this effect. In addition the $J / \psi$ is constrained to have a momentum in the centre of mass frame of between 1.47 and $1.90 \mathrm{GeV} / \mathrm{c}$. This cut is $95 \pm 1 \%$ efficient for signal $J / \psi$, but is $18 \pm 3 \%$ efficient for candidates from generic $B$ meson, and $14 \pm 2 \%$ for those from the $q \bar{q}$ continuum. The distributions for these quantities are shown in figures 5-4 and 5-5. A cut is then made on the probability that the tracks originated from a common vertex. The BABAR package RecVtx [77] is used for this purpose. This probability is required to be greater than $10^{-3}$. This cut is $93 \pm 1 \%$ efficient for signal $J / \psi$, while rejecting a further $25 \pm 5 \%$ of fake $J / \psi$ from both generic $B$ decays and for continuum background. Some efficiency is lost again due to bremsstrahlung in the electron decay mode. 


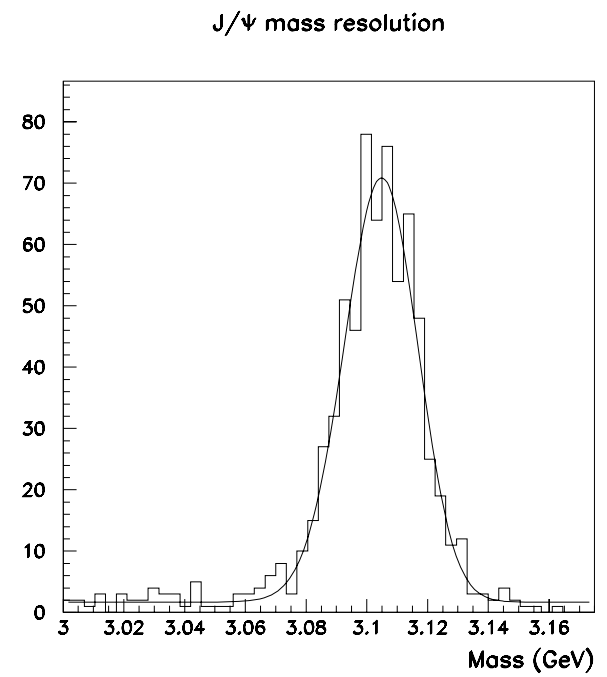

Figure 5-3. $J / \psi$ mass resolution. The fit is to the Novosibirsk function (see chapter 4) to account for the lower mass caused by electron bremsstrahlung. The width is $12.1 \mathrm{MeV}$.

\subsection{Reconstructing the $K_{S}^{0}$}

The $K_{S}^{0}$ has a lifetime of $8.934 \pm 0.008 \times 10^{-11} \mathrm{~s}$, and in the lab a typical momentum of approximately $3 \mathrm{GeV}$, giving it a flight length of around $8 \mathrm{~cm}$. The technique to account for this long flight length, described for the ideal case of four isolated photons in [81], is used and adapted for the case of merged $\pi^{0} \mathrm{~s}$, as will now be described.

A complete set of $\pi^{0}$ candidates is formed from combinations of all pairs of clusters consistent with an electromagnetic shape in the calorimeter whose invariant mass lies in the range $100 \mathrm{MeV} / \mathrm{c}$ to $145 \mathrm{MeV} / \mathrm{c}$, and all single clusters consistent with a merged $\pi^{0}$ shape, as described in chapter 4. Consistency is demanded at the $0.1 \%$ confidence level. The invariant mass for cluster pairs is shown in figure 5-6. $\pi^{0}$ pairs are then combined to form candidate $K_{S}^{0} \mathrm{~s}$, which are at this stage required to satisfy $380 \mathrm{MeV} / \mathrm{c}^{2}<\mathrm{m}_{\mathrm{K}_{\mathrm{S}}^{0}}<550 \mathrm{MeV} / \mathrm{c}^{2}$, where the mass is calculated at the decay point of the $J / \psi$. These cuts are necessarily loose in order to accommodate the error in their calculation due to the flight length. 


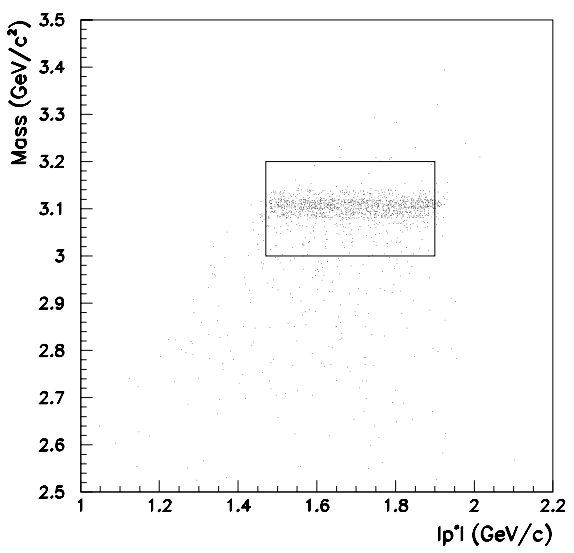

Figure 5-4. Mass and centre of mass momentum distribution for signal $J / \psi$ candidates from 2000 simulated events.
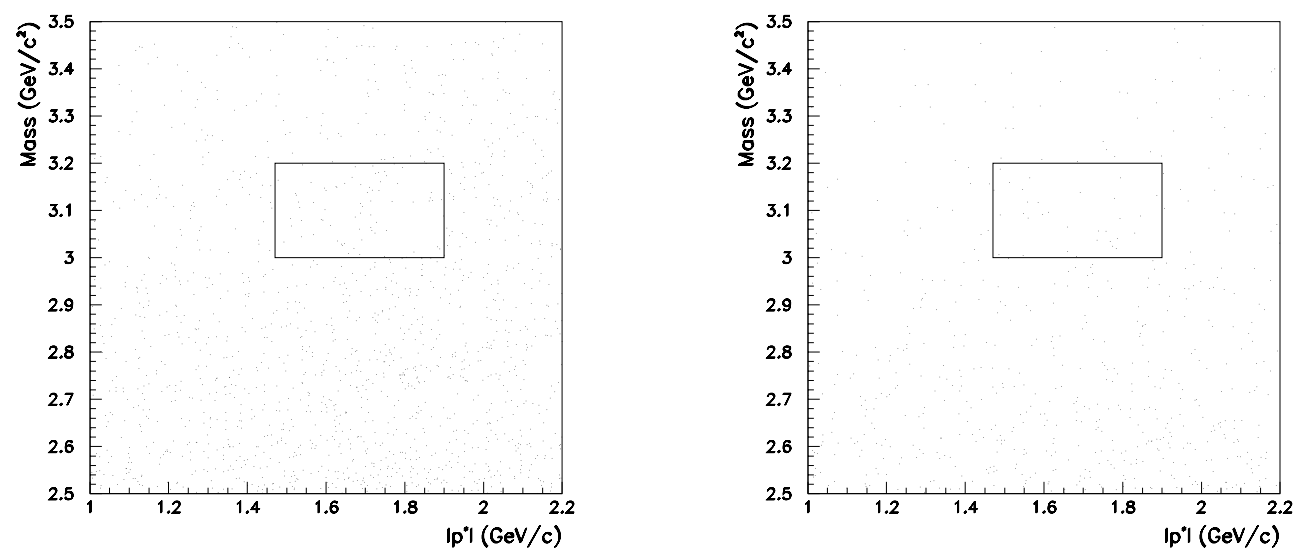

Figure 5-5. Fake $J / \psi$ mass and momentum distributions from (left) continuum and (right) generic $B$ meson decays. 10000 events were simulated. 
The reconstructed $J / \psi$ momentum and the assumed momentum of the $B$ meson are then used to estimate the $K_{S}^{0}$ direction. The small transverse momentum of the $B$ is ignored. Points along this direction are considered from -2.0 to $+5.0 \tau_{K S}^{0}$ in steps of $0.1 \tau_{K S}^{0}$.

At each point, if the two $\pi^{0}$ s constituting the candidate $K_{S}^{0}$ each decayed into isolated photons, the invariant mass of each cluster pair is calculated. The position at which the invariant mass is closest to the $\pi^{0}$ mass is used for each pair. The $K_{S}^{0}$ candidate is considered if the absolute
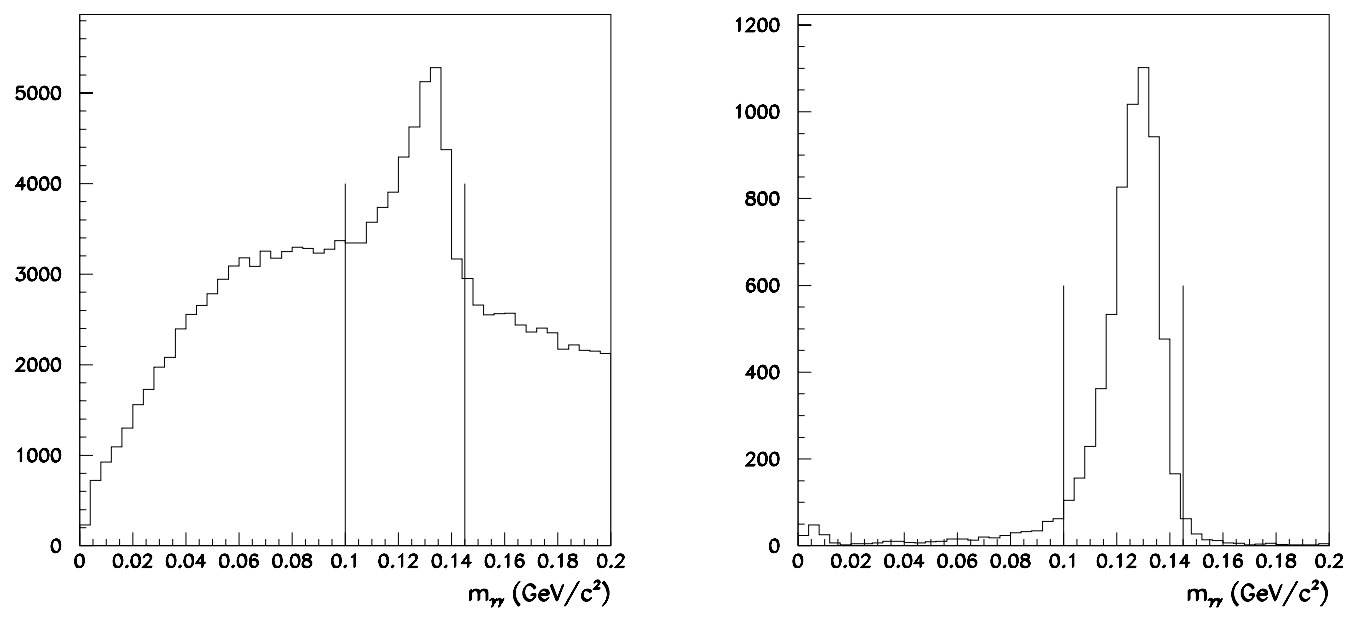

Figure 5-6. Two cluster invariant mass distribution for all cluster pairs (left) and for signal cluster pairs (right).

difference in these times for the two $\pi^{0}$ candidates is less than $3 \tau_{K_{S}^{0}}$ and the invariant masses of the $\pi^{0} \mathrm{~s}$ so formed lie in the range $110 \mathrm{MeV} / \mathrm{c}^{2}<\mathrm{m}_{\pi^{0}}<160 \mathrm{MeV} / \mathrm{c}^{2}$. Any candidate whose best lifetime falls at either end of the range described above is also rejected. The distributions for the best lifetime and absolute difference in lifetimes are shown in figure 5-7. Approximately 2/3 of $K_{S}^{0}$ decays in this channel give 4 isolated photons. The $K_{S}^{0}$ decay point is taken as the mean of the two best pion $\pi^{0}$ decay points. The $K_{S}^{0}$ candidate itself is constructed by combining the four photon clusters at this point.

If one of the $\pi^{0}$ s constituting the candidate $K_{S}^{0}$ is a merged $\pi^{0}$, the invariant mass of the cluster pair forming the other $\pi^{0}$ is calculated at each point. The point at which the mass difference of the cluster pair and the $\pi^{0}$ mass is minimal is kept, again providing that it does not lie at the extremes of the range described and that the mass of the $\pi^{0}$ so formed lies in the above range used for the case of 4 isolated photons. Almost all of the remaining 1/3 of $K_{S}^{0}$ candidates have one merged and 

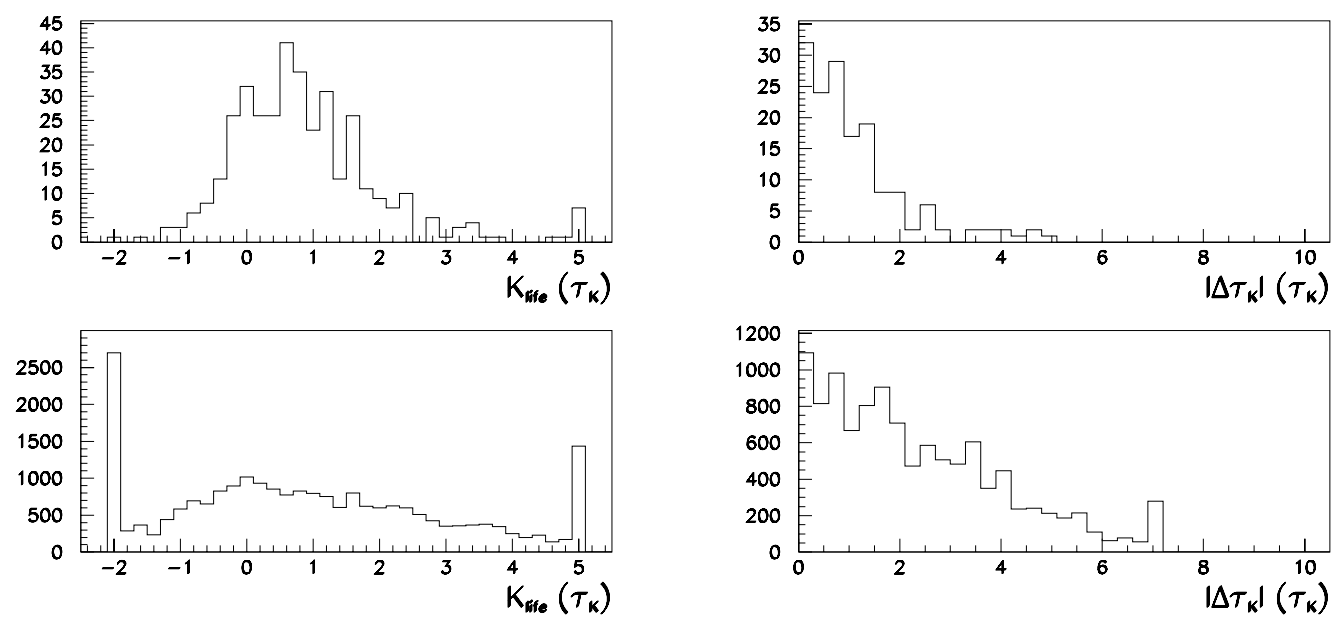

Figure 5-7. (Left) Distribution of the $K_{S}^{0}$ decay points as determined by the formation of the best $\pi^{0}$ mass for a cluster pair. The top plot is for $\pi^{0} s$ coming from signal $K_{S}^{0} s$, the lower from fake $K_{S}^{0}$ candidates. The spikes at -2 and +5 in the lower plot are caused by those candidates for which a best mass could not be determined between these times. (Right) Distribution of the absolute difference between the two decay points determined for each candidate. Again the top plot is for signal $K_{S}^{0}$, and the lower one for fake $K_{S}^{0}$ candidates. The small spike at 7.0 is due to those candidates whose best decay points were at the extremes of the range described above.

one unmerged $\pi^{0}$. The $K_{S}^{0}$ candidate is constructed in this case by combining the three clusters, where the merged $\pi^{0}$ cluster is assigned the $\pi^{0}$ mass.

If both of the $\pi^{0} \mathrm{~s}$ are merged, then the invariant mass of the pair is calculated at each point. The point at which this is closest to the $K_{S}^{0}$ mass is chosen. Again if this point is at the extremes of the range described the candidate is rejected. No constraint is imposed on the $K_{S}^{0}$ mass at this point. The fraction of $K_{S}^{0}$ decays falling into this category is less than $1 \%$.

The centre of mass momentum is then calculated for the $K_{S}^{0}$ candidate constructed as described above. The resulting distribution is shown in figure 5-8. The candidate is then required to have a centre of mass frame momentum lying in the range $1.4 \mathrm{GeV} / \mathrm{c}<\left|\mathrm{p}^{*}\right|<2.0 \mathrm{GeV} / \mathrm{c}$. This cut is $99.7 \%$ efficient for signal $K_{S}^{0}$, and rejects over $90 \%$ of combinatoric background. The $K_{S}^{0}$ mass of the candidate (shown in figure 5-9) is calculated at each point and the resulting overall mass resolution was found to be $15.7 \mathrm{MeV} / \mathrm{c}^{2}$. The effect of using only one $\pi^{0}$ decay point for the case 

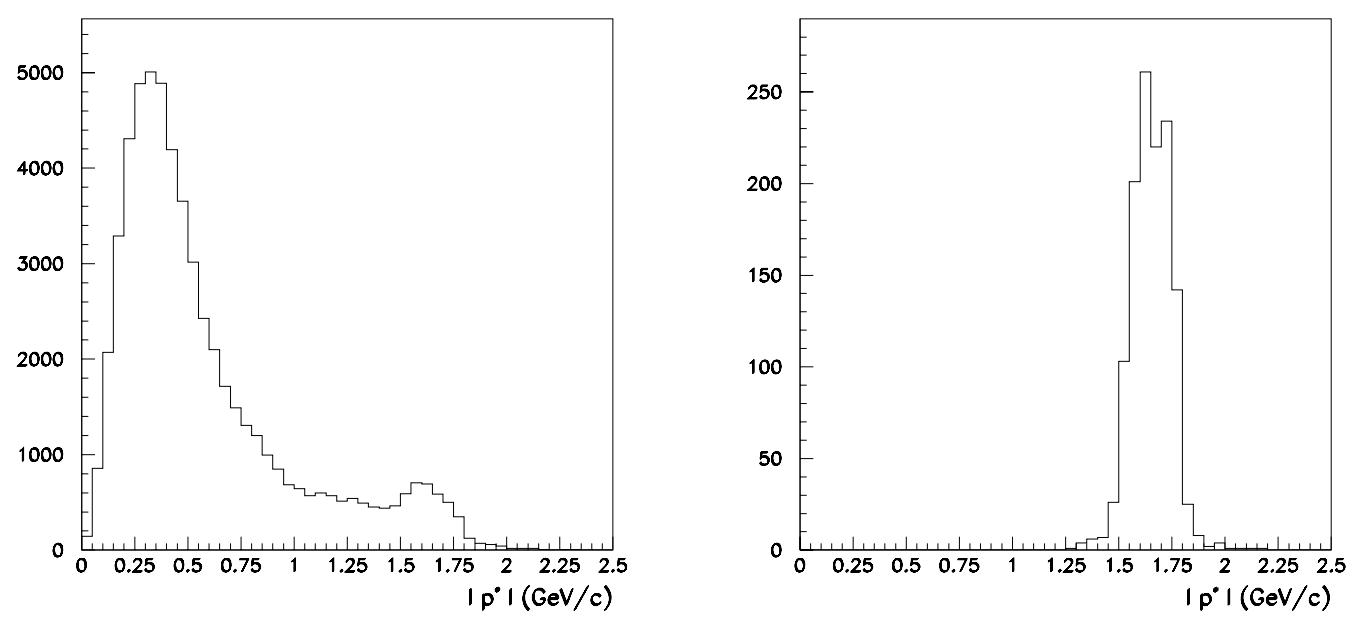

Figure 5-8. Distribution of centre of mass momentum of (left) all $K_{S}^{0}$ candidates and (right)

signal candidates.

of one merged $\pi^{0}$ and two isolated photons introduces a small bias to higher masses, as no account is made of the resulting $K_{S}^{0}$ mass when deciding on the decay point. A full kinematic fit including a constraint on the $K_{S}^{0}$ mass could be used in this case, but a widening of the $K_{S}^{0}$ mass window allowed was found to be sufficient. Observed $K_{S}^{0}$ candidates were kept if the mass was in the range $440 \mathrm{MeV} / \mathrm{c}^{2}<\mathrm{m}_{\mathrm{K}_{\mathrm{S}}^{0}}<560 \mathrm{MeV} / \mathrm{c}^{2}$.

\subsection{Reconstructing the $\mathrm{B}^{0}$}

$\mathrm{B}^{0}$ candidates are constructed from all possible pairs of $J / \psi$ and $K_{S}^{0}$ candidates reconstructed in the way described above. Each $J / \psi$ candidate is set to the the $J / \psi$ mass while preserving the measured momentum. Similarly each $K_{S}^{0}$ candidate is set to the $K_{S}^{0}$ mass while preserving its energy. $\mathrm{B}^{0}$ candidates so formed are required to lie in the mass range $5.0 \mathrm{GeV} / \mathrm{c}^{2}<\mathrm{m}_{\mathrm{B}^{0}}<$ $5.5 \mathrm{GeV} / \mathrm{c}^{2}$ and to have a centre of mass momentum of between $0.2 \mathrm{GeV} / \mathrm{c}^{2}$ and $0.5 \mathrm{GeV} / \mathrm{c}^{2}$.

This procedure yields on average 1.9 of $\mathrm{B}^{0}$ candidates per signal event, due mainly to the combinatorial background arising from the $K_{S}^{0}$ reconstruction procedure. In each event, the candidate closest to the $\mathrm{B}^{0}$ mass is kept. In just under $40 \%$ of events, this results in an incorrect $K_{S}^{0}$ candidate making up the $\mathrm{B}^{0}$. However, it is the measurement of the $B$ decay vertex that is required (see 

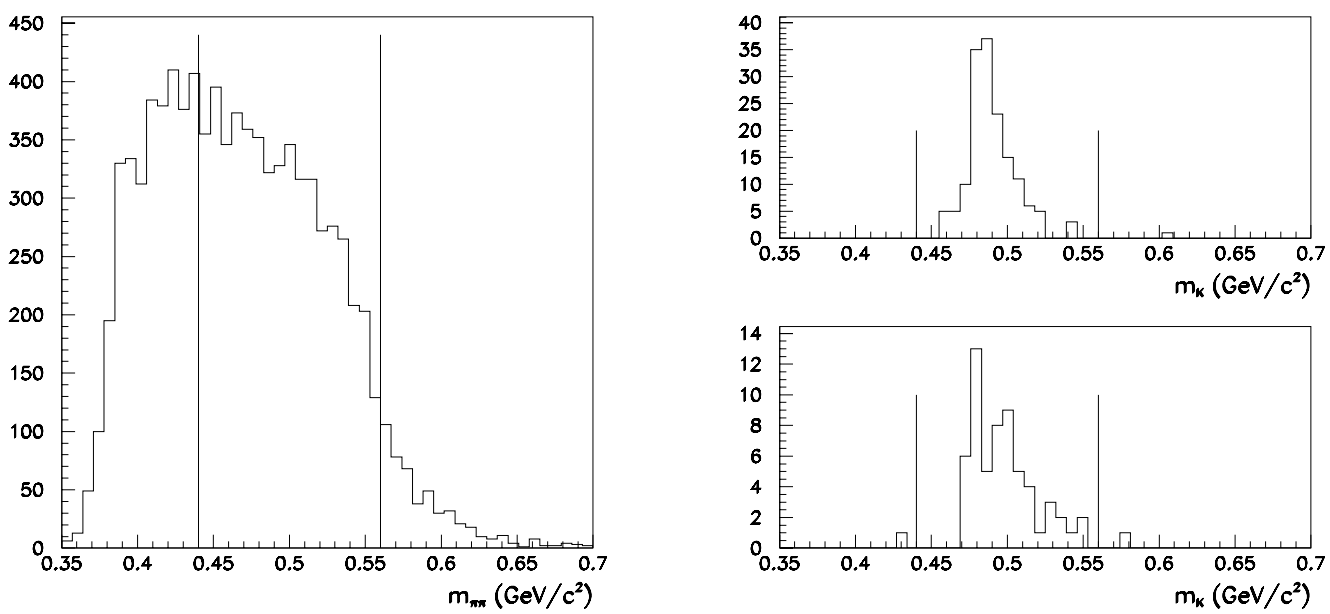

Figure 5-9. $\quad K_{S}^{0}$ mass distribution calculated at the decay vertex for non-signal (left) and signal (right) combinations.

section 5.6.1), and this position comes from the $J / \psi$ decay point. Therefore the inclusion of such a candidate is valid, providing the correct $J / \psi$ has been used. In events where a signal candidate is present, an incorrect $J / \psi$ candidate is selected by this method in less than $0.2 \%$ of events.

The overall reconstruction efficiency to reconstruct a $\mathrm{B}^{0}$ candidate with the correct $J / \psi$ is found to be $25.0 \pm 0.7 \%$, where the error quoted is purely statistical.

\subsection{Backgrounds}

\subsubsection{Combinatorial backgrounds and $J / \psi K_{L}^{0}$}

As discussed above, an average of 0.9 fake $B$ candidates are observed per event. It is essential to establish their origin since, if their origin is independent of the signal channel, events from the decay $\mathrm{B}^{0} \rightarrow J / \psi K_{L}^{0}$ reconstructed with a fake $K_{S}^{0}$ in place of the $K_{L}^{0}$ would allow for a large background of opposite $\mathrm{CP}$ asymmetry to the $K_{S}^{0}$ channel.

The composition of fake $B$ candidates is shown in figure 5-10. Approximately $2 \%$ originate from combinations of photons that do not come from the $K_{S}^{0}$, while less than $1 \%$ of these have the 


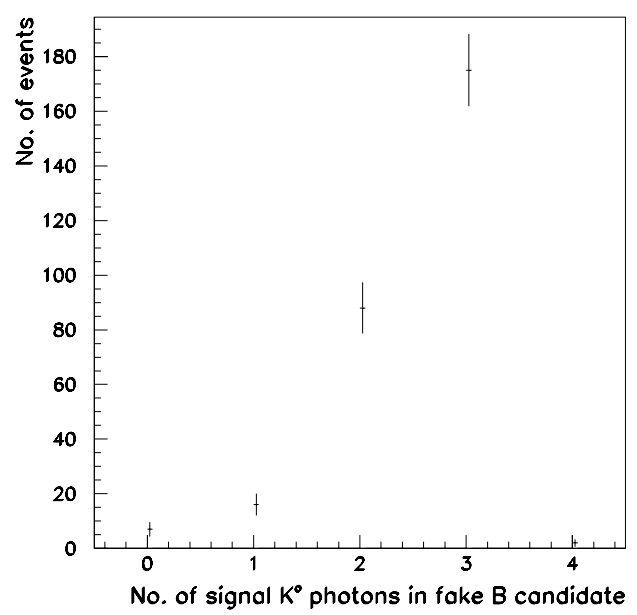

Figure 5-10. Origin of the photons in fake $B$ candidates in 2000 signal events. The number of photons that originated from the signal $K_{S}^{0}$ and subsequently used to construct the $B$ candidate is shown. Those candidates with 4 such photons have the correct $K_{S}^{0}$ but a fake $J / \psi$. Candidates with 0 such photons have the $K_{S}^{0}$ constructed entirely from photons from the other $B$ meson decay in the event. It can be seen that the large majority of events require at least one photon from the signal $K_{S}^{0}$ in order to fake a $B$ candidate.

correct $K_{S}^{0}$ but an incorrect $J / \psi$. The selection of the candidate whose mass is closest to the $B$ mass reduces these contributions further. The high fraction of combinatoric background candidates containing some fraction of the signal $K_{S}^{0}$ indicates that the $\mathrm{B}^{0} \rightarrow J / \psi K_{L}^{0}$ channel, which would otherwise present a significant physics background if a fake $K_{S}^{0}$ were available to combine with the $J / \psi$ from this decay, will not give a significant contribution to the final event sample.

\subsubsection{Physics backgrounds}

Previous studies [81] using the $B_{A} B_{A R}$ fast Monte Carlo, Aslund, indicate that the background from $\mathrm{B}^{0} \rightarrow J / \psi X$, where $X$ is anything except a $K_{S}^{0}$, is dominant over that from other sources (semileptonic decays of the $\mathrm{B}^{0}, q \bar{q}$ continuum) by a factor of 3. $6000 \mathrm{~J} / \psi X$ events were passed through the above analysis, where the $J / \psi$ was forced to decay into leptons. One event was observed to pass the cuts. At the $95 \%$ confidence level, this corresponds to a signal to background ratio of 10:1. 


\begin{tabular}{|c|c|}
\hline $\begin{array}{c}\text { Cluster threshold } \\
(\mathrm{MeV})\end{array}$ & Relative efficiency \\
\hline 20 & 0.98 \\
30 & 0.95 \\
40 & 0.89 \\
50 & 0.83 \\
\hline
\end{tabular}

Table 5-1. Relative reconstruction efficiency for the channel $B \rightarrow J / \psi K_{S}^{0} \rightarrow l^{+} l^{-} \pi^{0} \pi^{0}$ as a function of cluster energy cut at nominal machine background

The reconstruction efficiency for signal events using the fast Monte Carlo is found to be optimistic by approximately $30 \%$. The reconstruction efficiency for $\mathrm{B}^{0} \rightarrow J / \psi X$ found here is consistent with this factor: the fast Monte Carlo study indicates an efficiency of $7 \times 10^{-4}$, the above figures indicate an efficiency of $5.5 \times 10^{-4}$ at the $95 \%$ confidence level. No significant deviation therefore is expected from the observation that $\mathrm{B}^{0} \rightarrow J / \psi X$ is the dominant background for this channel.

\subsubsection{Machine backgrounds}

The soft-photon background caused by the lost beam particle collisions with the beam pipe and interaction region magnets causes a large combinatoric background which can be decreased by increasing the energy threshold required for a calorimeter cluster to be included in the analysis. 2000 signal event samples were simulated with overlaid machine background at nominal levels. These were then reconstructed with cluster thresholds of 20 (standard), 30, 40 and $50 \mathrm{MeV}$. The overall relative $B$ reconstruction efficiency is shown in table 5-1 where the figures are normalised to the reconstruction efficiency obtained in the case of no machine background. Once the cluster inclusion threshold is raised above $30 \mathrm{MeV}$, the dominant effect is the exclusion of signal clusters. A small effect is observed for higher background whereby candidates which would otherwise be excluded from the final sample are kept due to the additional energy attributed to signal clusters by machine background photons. Though small, this is an undesirable effect and indicates a lack of proper calibration at the higher background level. Such effects will be properly accounted for once the running conditions are known. At present, the calibration assumes a zero background level. 


\section{6 $C P$ reach}

Measuring the $C P$ violating asymmetry $a_{f}(t)$ in equation 5.1 requires knowledge of the proper time $t$ between the decay of the $B$ meson decaying to $J / \psi K_{S}^{0}$ (the $C P B$ ) and the other $B$ meson from the $\Upsilon(4 \mathrm{~S})$ decay (the $\operatorname{tag} B$ ), and the establishment of the $b$ quark flavour of the tag $B$ itself.

\subsubsection{Measuring $\Delta t$}

The measurement of the proper time between the decays requires that the position of the decays of the two $B$ mesons be established. The maximum transverse momentum of the $B$ mesons is $300 \mathrm{MeV} / \mathrm{c}$. The boost of the $\Upsilon(4 \mathrm{~S})$ at $B A B A R$ means that this component may be disregarded, and consequently only the measurement of the $z$ distance between the two decays is required.

The $C P B$ decay vertex is given by the $J / \psi$ decay vertex. This vertex is constructed using the RecVtx package, and the resulting resolution is shown in figure 5-11.

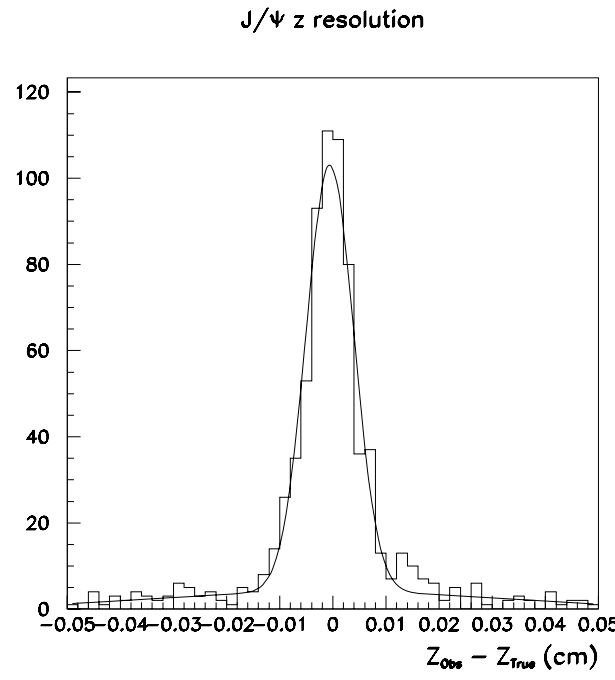

Figure 5-11. The $z$ resolution of the $J / \psi$ vertex. The fit is to two Gaussians, the upper having a width of $45 \mu \mathrm{m}$, the lower $320 \mu \mathrm{m}$. The ratio of areas is 3.5:1 
For each reconstructed $\mathrm{B}^{0}$ candidate in the signal channel, the remaining tracks in the event are passed to the $B_{A B A R}$ standard tag-side vertexing algorithm [82]. The outline of this algorithm is summarised here.

Tracks used to reconstruct the signal $\mathrm{B}^{0}$ are discarded. Of the remaining tracks, the default identified muon candidates are examined and any tracks retained to construct a tag $B$ candidate. If no such tracks are found, the default electron, charged pion and charged kaon candidates are examined in turn. The $y$ co-ordinate of the $J / \psi$ vertex is not used as the resolution on this point (see figure 5-12) is worse than the projected beam spread in the $y$ direction (see section 2.2.4) and

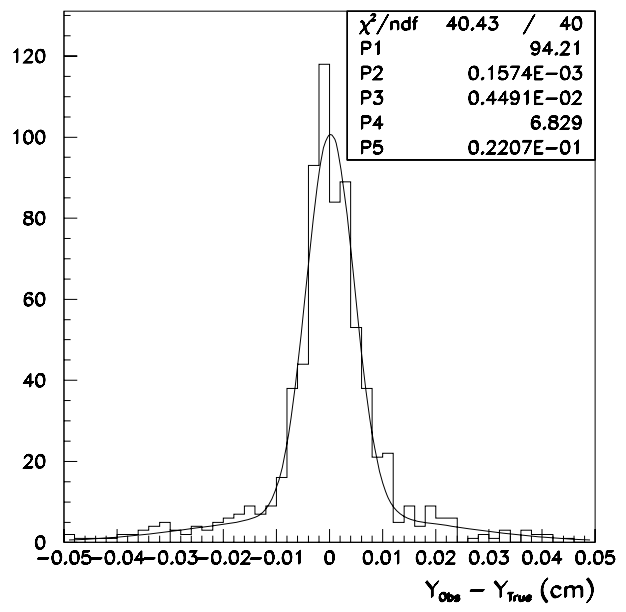

Figure 5-12. $J / \psi$ vertex resolution in the $y$ direction. A two Gaussian fit is used and the resulting widths are 45 and $221 \mu \mathrm{m}$ with area ratios 20:3. This is considerably larger than the vertical beam size.

so the impact parameter (distance of closest approach) of these tracks to a line in the $z$ direction through the point $\left(x, y_{\text {beam }}\right)$ is calculated, where $x$ is the co-ordinate of the $J / \psi$ vertex and $y_{\text {beam }}$ was set to zero in the Monte Carlo used. The $z$ co-ordinate of the point of distance of closest approach in the $x, y$ plane is then used.

Cuts on angular acceptance, vertexing probability and invariant mass of the resulting tag $\mathrm{B}$ candidate improve resolution on this vertex. However, the overall $C P$ reach is dominated by the low statistical power of the channel and no such cuts were imposed. 


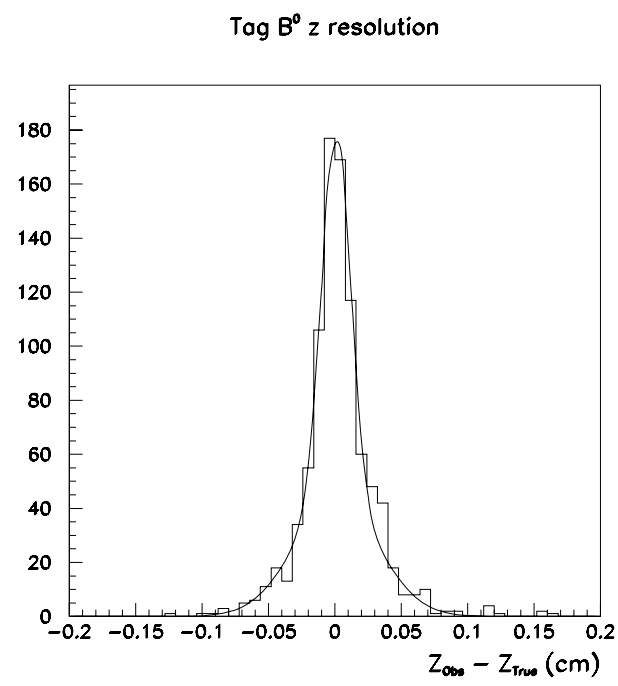

Figure 5-13. Tag side vertex resolution. A two Gaussian fit yields widths of $108 \mu \mathrm{m}$ and $316 \mu \mathrm{m}$,

with an area ratio 1.1:1.0.

The obtained tag-side vertex resolution is fit to two Gaussians. The widths are $108 \mu \mathrm{m}$ and $316 \mu \mathrm{m}$, with an area ratio 1.1:1.0. (see figure 5-13). No loss of efficiency is experienced in obtaining the vertex for the tag side.

\subsubsection{Determination of the $b$ quark flavour}

A sophisticated multi-variable analysis tool, CORNELIUS [83], is in standard $B_{A B A R}$ use to provide information on the $b$ quark flavour. The general approach is now described.

The determination of the $b$ quark flavour is performed by looking for charged decay products whose sign is correlated with the $b$ quark flavour. The most useful processes for this are $b \rightarrow$ $c W^{-}, c \rightarrow W^{+} s, s \rightarrow K^{-}$, and $b \rightarrow W^{-} X, W^{-} \rightarrow l^{-} \nu$, where the sign resulting kaon or lepton gives the $b$ flavour. The identification of kaons uses the DIRC particle identification device and $d E / d x$ information for lower momentum particles. The identification of leptons in semi-leptonic decays uses several variables (transverse momentum, transverse energy, missing momentum). The overall efficiency $\epsilon$ for this determination is currently expected to be approximately $40 \%$ for leptons and $20 \%$ for kaons ([31], chapter4). Cascade decays of the resulting charm or $W$ can give an $s$ quark or lepton in the final state which has the wrong sign. The result of a fraction $\omega$ 
of events where the wrong sign is attributed to the $b$ is a dilution of the tagging purity by a factor $(1-2 \omega)^{2}$. The overall resolution on a given $C P$ asymmetry is degraded by a factor $\left(\epsilon(1-2 \omega)^{2}\right)^{-\frac{1}{2}}$. Determinations of the factor $(1-2 \omega)^{2}$ currently estimate this to be in the range $40 \%$ (for channels with either a lepton or a kaon), and $60 \%$ (for channels with both a kaon and a lepton). The overall factor $\left(\epsilon(1-2 \omega)^{2}\right)^{-\frac{1}{2}}$ averaged across all channels is approximately $35 \%$.

\subsection{3 $C P$ reach}

In its most simple form, the asymmetry $a_{f}(t)$ can be determined by enumerating the number of events $N(\bar{N})$ which have a flavour tag of $B(\bar{B})$ in a particular proper time difference interval $\Delta t$. The asymmetry for that interval is then determined easily:

$$
a_{f}(\Delta t)=\left.\frac{N-\bar{N}}{N+\bar{N}}\right|_{\Delta t}
$$

from which a fit to the overall asymmetry can be made to determine the quantity $\sin 2 \beta$. The overall $\mathrm{CP}$ reach is a function of the $\mathrm{CP}$ violating angle itself, the overall $z$ resolution, and the $\mathrm{B}$ mixing parameter $x=\Delta m / \Gamma$. The expected error for $N_{s}$ signal events with an asymmetry $A_{s}$ with $N_{b}$ background events with an asymmetry $A_{b}$ is given by [31], p218,

$$
\sigma_{\sin 2 \beta}=\frac{\sigma_{0}\left(\sin 2 \beta, x_{d}, \sigma_{z}\right)}{\sqrt{N_{s}} \sqrt{\epsilon(1-2 \omega)^{2}}} \frac{\sqrt{1+N_{b} / N_{s}}}{1+\left(A_{b} N_{b} / A_{s} N_{s}\right)} .
$$

The value of $\sigma_{0}$, which can be thought of as the contribution to the error from a single event with perfect $b$ quark flavour determination and no background, is drawn from tabulated values [84]. For a nominal years running at an integrated luminosity of $30 \mathrm{fb}^{-1}$, the expected error on a $\sin 2 \beta$ value of 0.7 is 0.34 . The equivalent figure for the charged pion decay mode of the $K_{S}^{0}$ is 0.10 [31].

\subsection{Chapter Summary}

The reconstruction of the decay $B^{0} \rightarrow J / \psi K_{S}^{0} \rightarrow l^{+} l^{-} \pi^{0} \pi^{0}$ has been described. The overall efficiency for obtaining a candidate $\mathrm{B}^{0}$ with the correct $J / \psi$ is $25.0 \pm 0.7 \%$. It has been shown that such candidates are valid to measure the angle $\beta$ in the unitarity triangle even in the cases where an incorrect $K_{S}^{0}$ has been reconstructed. Relevant backgrounds from physics channels have been evaluated and shown to contribute no more than a $10 \%$ contamination. The effect of machine background at nominal levels has been evaluated. Efficiency falls by approximately $17 \%$ if the cluster energy threshold described in chapter 4 needs to be raised to $50 \mathrm{MeV}$. A discussion of the techniques necessary to make a $C P$ measurement has been given, and the overall $C P$ reach for this channel evaluated to give an error on $\sin 2 \beta$ of 0.34 after 1 year's running at design luminosity corresponding to the observation of $68 \pm 8$ events per year. This is to be compared to an error on $\sin 2 \beta$ of 0.10 from the charged pion decay of the $K_{S}^{0}$. 


\section{Conclusions}

Certain decays of neutral $B$ mesons are expected to exhibit a large $C P$ asymmetry. The $B A B A R$ experiment, to be operated at the PEP-II asymmetric storage ring, is designed to make precision measurements of this phenomenon, as well as determinations of non- $C P$ violating parameters in the $B$ system, in order to over constrain the unitarity triangle to allow for a test of the accommodation of the $C P$ violation phenomenon in the standard model.

The electromagnetic calorimeter is a key component in the BABAR experiment. The chosen technology of Thallium doped Caesium Iodide crystals in a carbon fibre support will provide unprecedented resolution. It is crucial that all elements from readout to reconstruction software maximise this physics potential.

A framework of software has been developed which takes the basic readout from the front end electronics and converts it through several stages to a form suitable for physics analysis. This software employs new techniques to maximise physics potential. Firstly, the BABAR standard language of $\mathrm{C}++$ is adopted. This allows for the employment of Object Oriented analysis and design, which in turn allows for more robust software and decreased maintenance. Secondly, the application of object oriented design has allowed the construction of a complete chain of reconstruction software for the calorimeter-the finding of clusters, the division of clusters into potential electromagnetic maxima, the matching of energy deposits to reconstructed tracks, particle identification and global calibration - in such a way as to provide a functional framework which is at the same time completely extensible for increased physics capability.

In addition, the simulation software necessary to recreate the front end electronics output given a known energy deposit in each crystal has been written. This incorporates a simulation of the matched digital filter used in crystal energy extraction, and this has been shown to be effective in improving resolution and decreasing the number of extraneous energy deposits due to machine background.

The software has been employed in a study of the $C P$ violating channel $\mathrm{B}^{0} \rightarrow J / \psi K_{S}^{0} \rightarrow$ $l^{+} l^{-} \pi^{0} \pi^{0}$. Particle identification for photons and merged $\pi^{0} \mathrm{~s}$, necessary to reconstruct the $\pi^{0} \mathrm{~s}$ in the energy range for this decay was developed and employed. The complete software chain from front end electronics simulation to calibration was demonstrated to be functional. The overall $\mathrm{B}^{0}$ reconstruction efficiency for this channel is projected to be $25 \pm 0.7 \%$, which results in an uncertainty on $\sin 2 \beta$ of 0.34 after a nominal year's running $\left(30 \mathrm{fb}^{-1}\right)$. 


\section{References}

[1] J.H. Christenson, J.W. Cronin, V.L. Fitch and R. Turlay, Phys. Rev. Lett. 13 (1964) 138.

[2] A. D. Sakharov, ZhETF Pis. Red. 5 (1967) 32; JETP Lett. 5 (1967) 24.

[3] M. Kobayashi and T. Maskawa, Prog. Th. Phys. 49 (1973) 652.

[4] Z. Xing, Phys. Rev. D 53(1996) 204

[5] CP Violation, ed. L. Wolfenstein, from the series "Current Physics Sources and Comments" and references therein (North Holland, Amsterdam, 1989).

[6] L. Wolfenstein, Ann. Rev. Nucl. Part. Sci. 36137

[7] I. Manelli, Rivista del Nuovo Cimento 8, No. 7, 1

[8] K. Kleinknecht, Ann. Rev. Nucl. Part. Sci. 261

[9] C. Caso et al., The Particle Data Group, The European Physical Journal C3 (1998) 1

[10] G. D. Barr et al., NA31 Collaboration, Phys. Lett. B 317 (1993) 222

[11] L. K. Gibbons et al., E731 Collaboration, Phys. Rev. Lett. 70 (1993) 1203.

[12] I. I. Bigi, V. A. Khoze, N. G. Uraltsev and A. I. Sanda, in CP Violation, ed. C. Jarlskog (World Scientific, Singapore, 1992)

[13] H. Albrecht et. al., The ARGUS Collaboration, Phys. Lett. B, 192245

[14] M. Peskin and D. Schroeder, Introduction to Quantum Field Theory, AddisonWesley Publishing Company (1995), in particular the latter half of chapter 3

[15] R. D. Peccei in CP Violation, ed C. Jarlskog, (World Scientific, Singapore, 1989) p. 503

[16] M. Neubert, Int. Jour. Mod. Phys. A, 11, No. 23 (1996) 4173

[17] J. D. Richman, "Heavy Quark Physics and CP Violation", given at Les Houches summer school 1997, to appear in proceedings

[18] Cheng and Li, Gauge Theory in Elementary Particle Physics, Oxford University Press (1984)

[19] M. Kobayashi and T. Maskawa, Prog. Theor. Phys. 49, No. 2652

[20] C. Jarlskog, Z. für Phys. C. 29, pp. 491-497 and Phys. Rev. Lett. 551039.

[21] N. Cabbibo, Phys. Rev. Lett. 10, 531 (1963) 
[22] L.Wolfenstein, Phys. Rev. D. 39 No. 92381 and Phys. Rev. Lett. 511945

[23] I. Dunietz and J. L. Rosner, Phys.Rev. D. 34 No.5, 1404.

[24] I. I. Bigi and A. I. Sanda, Nucl. Phys. B. 19385 and Nucl. Phys. B. 28141

[25] X-G .He, Phys. Rev. D. 536326 and references therein.

[26] Y. Nir and Helen R. Quinn, Ann. Rev. Nucl. Part. Sci. 42 (1992) 211

[27] C. O. Dib, I. Dunietz, F. J. Gilman and Y. Nir, Phys. Rev. D 41 No. 5, 1522

[28] R. Godang et al., The CLEO Collaboration, Phys. Rev. Lett. 803456 (1998)

[29] M. Gronau and D. London, Phys. Rev. Lett. 653381 (1990)

[30] F. Parodi, P. Roudeau and A. Stocchi, "Constraints on the parameters of the $V_{C K M}$ matrix at the end of 1997", LAL 98-49 and given at ICHEP 98, to appear in proceedings

[31] D. Boutigny et al. The BABAR collaboration, "Physics at the BABAR experiment", eds P. F. Harrison and H. Quinn, (in prepraration)

[32] P. Paganini, F. Parodi, P. Roudeau and A. Stocchi, "Measurement of the $\rho$ and $\eta$ parameters of the $V_{C K M}$ matrix and perspectives", LAL 97-79

[33] J. P. Alexander et al. (The CLEO Collaboration)

[34] H. Schroder, “ $B \bar{B}$ Mixing”, in $B$ Decays, ed. S. Stone, World Scientific (1994)

[35] PEP-II, An Asymmetric B-Factory, Conceptual Design Report, SLAC418/LBL-PUB-5379/CALT-68-1869/UCRL-ID-114055/UC-IRPA-93-01

[36] P. J. Oddone, in Proceedings of the UCLA workshop: Linear collider $B \bar{B}$ Factory Conceptual Design, p. 423

[37] The Physics Program of a High Luminosity Asymmetric $B$ Factory at SLAC, ed. D. Hitlin, SLAC-353/LBL-PUB-5245/CALT-68-1588 (1989)

[38] Proc. of Workshop on Physics and Detector Issues for a High Luminosity Asymmetric B Factory at SLAC, ed. D. Hitlin, SLAC-373/LBL-30097/CALT68-1697 (1990)

[39] B Factories: The State of the Art in Accelerators, Detectors and Physics, ed. D. Hitlin, SLAC-400 (1992)

[40] A. Garren et al., in Proceedings of the 1989 IEEE Particle Accelerator Conference, Chicago (p. 1847)

[41] M. Sands, Physics of Electron Storage Rings, SLAC-121 (1970) 
[42] S. Henderson, CLNS 97/1528, (1997)

[43] D. Coupal, BABAR Note \# 210 (1995), BABAR Note \# 178 (1994)

[44] K. L. Brown and F. C. Iselin, TURTLE (Trace Unlimited Rays Through Lumped Elements), CERN 74-2 (1974) and D. C. Carey et al., Decay TURTLE, SLAC-246 (1982)

[45] GEANT Detector Description Tool, v3.21, CERN Program Library W5103, CERN (1994), R. Brun et al., Simulation program for particle physics experiments, GEANT : user guide and reference manual, CERN DD-78-02

[46] A. Snyder, The Effect of Vertex Cuts on CP reach, BABAR Note \# 177 (1994)

[47] P. Gaidarev et al. (The CLEO Collaboration) Nucl. Instr. Methods A 379 (1996) 396, B. Mours et al. (The ALEPH Collaboration) Nucl. Instr. Methods A 379 (1996) 101, A. McKemey et al. Nuo. Cim. 109A (1996) 1027. For an update on the performance of the latter two devices, see D. Creanza et al. (The ALEPH Collaboration) Nucl. Instr. Methods A 409 (1998) 157 and N.B. Sinev et al. (The SLD Collaboration) 409 (1998) 243

[48] D. Coupal et al. The $B_{A} B_{A R}$ drift chamber electronics group, Drift Chamber technical note TNDC-97-68 v4.0.

[49] W. W. M. Alison and P. R. S. Wright, The Physics of Charged Particle Identification: $\mathrm{dE} / \mathrm{dx}$, Čerenkovand Transition Radiation, in Experimental Techniques in High Energy Physics, ed. T. Ferbel, Addison Wesley (1987)

[50] D. Boutigny et al., The BABAR Collaboration, Letter of Intent, SLAC-443 (1992)

[51] Y. Kubota et al., The CLEO Collaboration, Nucl. Instr. Methods A 32060 (1992)

[52] R. Santonico and R. Cardarelli, Nucl. Instr. Methods A 187 (1981), R. Cardarelli et al. Nucl. Instr. Methods A 263 (1988)

[53] See for example L. Antonazzi et al. (E771 collaboration), Nucl. Instr. Methods A 31592 (1992) , C. Bacci et al. (RD 5 collaboration) ibid. 102, A. Anotelli et al. (FENICE collaboration), Nucl. Instr. Methods A 33734 (1993)

[54] N. Dyce et al., The BABAR Trigger Group, "The BABAR Trigger System Design Requirements", V4.00 (1997)

[55] S. Gehrig, "Design and Simulated Performance of the Level 1 Trigger System”, BABAR Note \# 380 (1997) 
[56] G. Booch, Object Oriented Analysis and Design (2nd ed.), (Addison-Wesley, California, 1994)

[57] E. Gamma, R. Helm, R. Johnson and J. Vlissides, "Design Patterns", (Addision-Wesley, California, 1995)

[58] D. S. Humpherys, Analysis, Design and Synthesis of Electrical Filters, (Prentice Hall, New Jersey, 1970)

[59] E. A. Lee and D. G. Messerschmidt, Digital Communication, (Kluwer Academic Publishers, Boston, 1988)

[60] Y. Kubota et al. (The CLEO Collaboration), Nucl. Instr. Methods A 32066 (1992)

[61] J. Dowdell, BABAR Calorimeter DAQ system overview (BABAR Calorimeter internal note, August 1997), J. Dowdell, BABAR Calorimeter DAQ ADC Board Description, (BABAR Calorimeter internal note, May 1997), G. M. Haller and D. R. Freytag, "Analogue Floating Point BiCMOS Sampling Chip and Architecture of the $B_{A B} B_{A R}$ CsI Calorimeter Front End Electronics System at the SLAC B-Factory”, BABAR Note \# 285

[62] E. D. Frank, R. G. Jacobsen and E. Sexton-Kennedy, "Architecture of the BABAR Reconstruction System", presented at "Computing in High Energy Physics 1997", to appear in proceedings.

[63] W. H. Press et. al, "Numerical Recipes - the art of scientific computing", (Cambridge University Press, Cambridge, 1986)

[64] J. Back, "Track cluster matching for the Electromagnetic Calorimeter using Detector Model", BABAR Note \# 440 and "Track cluster matching for the Electromagnetic Calorimeter using a Simple Model”, BABAR Note \# 439

[65] The clustering algorithm was developed and written by S. Playfer and S. Gowdy of the Univeristy of Edinburgh.

[66] S. Gowdy and P. Strother, "BaBar EMC Reconstruction Code", internal document supplied in the EmcReco package of the BABAR offline software releases. Also available at http://www.slac.stanford.edu/BFROOT/dist/releases/newest/EmcReco/calreco.tex

[67] J. Izen, A. Snyder, M. Sokoloff and R. Waldi, "Some Statistics for Particle Identification”, BABAR Note \# 422, and references therein.

[68] E. D. Frank, "The ProxyDict mechanism”, BABAR note, in preparation

[69] Objectivity/DB, Object Oriented Database product supplied by Objectivity Inc., Mountainview, California 
[70] D. Boutigny et al., The BABAR Collaboration, The BABAR Technical Design Report, SLAC-R-95-457 (1995)

[71] T. Mattison, talk given at the July 1998 BABAR collaboration meeting.

[72] R. Seitz, "Describing Energy Deposits in CsI Crystals", BABAR Note \# 294

[73] N. J. Gunawardane, "Increasing the efficiency of the BaBar EMC using software", Imperial College internal report, 1998

[74] M. G. Bekishev and V. N. Ivanchenko, Nucl. Instr. Methods A 361138 (1995), A. De Angelis and P. Palazzi, Nucl. Instr. Methods A 28193 (1989), A. De Angelis and F. Mazzone, Nucl. Instr. Methods A bf 287397 (1990), F. Bianchi et al. (The Delphi FEMC group), Nucl. Instr. Methods A 279473 (1989)

[75] D. Antreasyan, "An algorithm for tagging photon and merged $\pi^{0}$ showers in the crystal ball", Crystal Ball note 321, 1983

[76] S. F. Schaffner and J. M. Losecco, "Object Oriented Tracking and Vertexing at BABAR", David Nathan Brown, "An Object Oriented Kalman Filter Tracking Algorithm", in proceedings of the CHEP 1997 conference, submitted to Comp. Phys. Comm.

[77] The RecVtx software package was written by John LoSecco, University of Notre Dame, Illinois

[78] P. F. Harrison, "Consistencies: definition and usage", Internal BABAR physics note, 1997

[79] P. Janot and F. Le Diberder, “Combining Limits”, CERN-PPE-97-053 (1997)

[80] C. P. Jessop et al. The CLEO Collaboration, Phys. Rev. Lett. 79 (1997) 45334537

[81] P. F. Harrison, "Study of $B^{0} \rightarrow J / \psi K_{S}^{0}$ and $B^{0} \rightarrow \rho^{ \pm} \pi^{\mp ”, ~ B A B A R ~ N o t e ~ \# ~} 218$

[82] D. Zanin, M. Bona, VtxTagBFinder code, documented in http://www.to.infn.it/esperimenti/babar/physics/ztag/zbest.html

[83] M. Benkebil et al. The BABAR Tagging Group. A most recent investigation of the performance of the tagging algorithms can be found in ref [31].

[84] F. LeDiberder, "Precision on $C P$ violation measurements and requirements on the vertex resolution", BABAR Note \# 34, 1990

[85] O. Nachtmann, Elementary Particle Physics, (Springer Verlag, Berlin, 1990)

[86] H. Schildt, "C++, The Complete Reference", 2nd Ed. (McGraw Hill, Berkeley, 1995) 
[87] M. Ellis and B. Stroustrup, "The Annotated C++ Reference Manual” (Addison Wesley, Reading, 1990) 


\section{The Wigner-Weisskopf formalism}

In this appendix the formalism describing the decay of elementary quantum mechanical states is described. The main result, namely the description of the time evolution of two states via a mass matrix is used extensively in the description of mixing of neutral $B$ mesons and $K$ mesons.

If some state, $|\Psi(t)\rangle$, is originally in some linear combination of unstable states $|\alpha\rangle$, which decay into some assembly of other states $|\beta\rangle$, then a general decomposition of $|\Psi(t)\rangle$ is as follows,

$$
|\Psi(t)\rangle_{S}=\sum_{\alpha} \psi_{\alpha}(t)|\alpha\rangle+\sum_{\beta} c_{\beta}(t)|\beta\rangle
$$

where the subscript $S$ indicates that the states are represented in the Schrödinger picture. The normalisation of this state dictates that any loss in population of the states $|\alpha\rangle$ will be matched by a rise in population of the states $|\beta\rangle$. At time $t=0$, the state $|\Psi(t)\rangle_{S}$ consists only of the original states, i.e.

$$
|\Psi(0)\rangle_{S}=\sum_{\alpha} \psi_{\alpha}(0)|\alpha\rangle
$$

This system will evolve according to the Schrödinger equation,

$$
i \frac{\partial|\Psi(t)\rangle_{S}}{\partial t}=\widehat{H}|\Psi(t)\rangle_{S}
$$

where $\widehat{H}=\widehat{H}_{0}+\widehat{H^{\prime}}, \widehat{H^{\prime}}$ being a perturbation of $\widehat{H}$ about $\widehat{H}_{0}$. In the case of the meson systems discussed below, the perturbative Hamiltonian relates to the weak force, whilst $\widehat{H}_{0}$ relates to the strong force. In the interaction picture (denoted by a subscript $I$ ), the relevant equations are

$$
\begin{aligned}
|\Psi(0)\rangle_{I} & =|\Psi(0)\rangle_{S} \\
|\Psi(t)\rangle_{I} & =e^{i \widehat{H}_{0} t}|\Psi(t)\rangle_{S} \\
& =\sum_{\alpha} a_{\alpha}(t)|\alpha\rangle+\sum_{\beta} b_{\beta}(t)|\beta\rangle \\
i \frac{\partial|\Psi(t)\rangle_{I}}{\partial t} & =\widehat{H^{\prime}}(t)|\Psi(t)\rangle_{I} \\
\widehat{H^{\prime}}(t) & =e^{i \widehat{H}_{0} t} \widehat{H}^{\prime} e^{-i \widehat{H}_{0} t} .
\end{aligned}
$$

If the states $|\alpha\rangle$ and $|\beta\rangle$ are such that

$$
\begin{aligned}
& \widehat{H}_{0}|\alpha\rangle=E_{0}|\alpha\rangle \\
& \widehat{H}_{0}|\beta\rangle=E_{\beta}|\beta\rangle,
\end{aligned}
$$


then the operator expansion of the expression A.5 is given by

$$
\begin{aligned}
\widehat{H^{\prime}}(t)= & \sum_{\alpha} \sum_{\alpha^{\prime}}|\alpha\rangle\left\langle\alpha \mid \alpha^{\prime}\right\rangle\left\langle\alpha^{\prime}\right| \\
& +\sum_{\alpha} \sum_{\beta} e^{i E_{0} t} e^{-i E_{\beta} t}|\alpha\rangle\langle\alpha \mid \beta\rangle\langle\beta| \\
& +\sum_{\alpha} \sum_{\beta} e^{i E_{\beta} t} e^{-i E_{0} t}|\beta\rangle\langle\beta \mid \alpha\rangle\langle\alpha| \\
& +\sum_{\beta} \sum_{\beta^{\prime}} e^{i E_{\beta} t} e^{-i E_{\beta^{\prime}} t}|\beta\rangle\left\langle\beta \mid \beta^{\prime}\right\rangle\left\langle\beta^{\prime}\right| .
\end{aligned}
$$

If one assumes that the final and initial states are all mutually orthogonal, then the equation (A.4) gives

$$
\begin{aligned}
& i \frac{\partial a_{\alpha}(t)}{\partial t}=\sum_{\alpha^{\prime}} a_{\alpha^{\prime}}(t)\left\langle\alpha\left|\widehat{H^{\prime}}\right| \alpha^{\prime}\right\rangle+\sum_{\beta} b_{\beta}(t) e^{i\left(E_{0}-E_{\beta}\right) t}\left\langle\alpha\left|\widehat{H^{\prime}}\right| \beta\right\rangle \\
& i \frac{\partial b_{\beta}(t)}{\partial t}=\sum_{\beta^{\prime}} b_{\beta^{\prime}}(t) e^{i\left(E_{\beta}-E_{\beta^{\prime}}\right) t}\left\langle\beta\left|\widehat{H^{\prime}}\right| \beta^{\prime}\right\rangle+\sum_{\alpha} a_{\alpha}(t) e^{i\left(E_{\beta}-E_{0}\right) t}\langle\beta|\widehat{H}| \alpha\rangle .
\end{aligned}
$$

The approximation made by Wigner and Weisskopf is that the first part of the latter equation makes no contribution, i.e. that the final states are stable with respect to the weak interaction to first approximation. If one then imposes that the initial state contains no component in the decay state space, i.e. that

$$
\begin{aligned}
a_{\alpha}(0) & =\psi_{\alpha}(0) \\
b_{\beta}(0) & =0
\end{aligned}
$$

then the solutions for the coefficients are

$$
b_{\beta}=-i \sum_{\alpha^{\prime}} \int_{0}^{t} e^{i t^{\prime}\left(E_{\beta}-E_{0}\right)}\left\langle\beta\left|\widehat{H^{\prime}}\right| \alpha^{\prime}\right\rangle a_{\alpha^{\prime}}(t) d t^{\prime},
$$

and, using this expression for the coefficient $b_{\beta}$,

$$
\begin{aligned}
a_{\alpha}(t)= & \psi_{\alpha}(0)-i \int_{0}^{t} \sum_{\alpha^{\prime}} a_{\alpha^{\prime}}\left(t^{\prime}\right)\left\langle\alpha\left|\widehat{H^{\prime}}\right| \alpha^{\prime}\right\rangle d t^{\prime} \\
& -\sum_{\alpha^{\prime}} \sum_{\beta^{\prime}} \int_{0}^{t} \int_{0}^{t^{\prime}} e^{i\left(E_{0}-E_{\beta}\right)\left(t^{\prime}-t^{\prime \prime}\right)}\left\langle\alpha^{\prime}\left|\widehat{H^{\prime}}\right| \beta\right\rangle\left\langle\beta\left|\widehat{H^{\prime}}\right| \alpha^{\prime}\right\rangle a_{\alpha^{\prime}}\left(t^{\prime \prime}\right) d t^{\prime \prime} d t^{\prime} .
\end{aligned}
$$

This integral is computed in, for example, [85]. The result is

$$
\begin{aligned}
a(t) & =\frac{1}{2 \pi i} \oint e^{\sigma t} \frac{1}{\sigma+i \mathcal{W}} \psi(0) d \sigma \\
& =e^{-i \mathcal{W} t} \psi(0),
\end{aligned}
$$


where the matrix $\mathcal{W}$ is given by

$$
\left.\mathcal{W}_{\alpha \alpha^{\prime}}=\left\langle\alpha\left|\widehat{\mathcal{H}^{\prime}}\right| \alpha^{\prime}\right\rangle+\sum_{\beta} \frac{\left\langle\alpha\left|\widehat{\mathcal{H}^{\prime}}\right| \beta\right\rangle\left\langle\beta\left|\widehat{\mathcal{H}^{\prime}}\right| \alpha^{\prime}\right\rangle}{\left(\mathcal{E}_{\prime}-\mathcal{E}_{\beta}\right)}-\right\rangle \pi \sum_{\beta} \delta\left(\mathcal{E}_{\prime}-\mathcal{E}_{\beta}\right)\left\langle\alpha\left|\widehat{\mathcal{H}^{\prime}}\right| \beta\right\rangle\left\langle\beta\left|\widehat{\mathcal{H}^{\prime}}\right| \alpha^{\prime}\right\rangle .
$$

If one now returns to the Schrödinger picture,

$$
\begin{aligned}
|\Psi(t)\rangle_{S} & =e^{-i H_{0} t}|\Psi(t)\rangle_{I} \\
& =\sum_{\alpha} e^{-i\left(E_{0}+\mathcal{W}\right) t} \psi(0)|\alpha\rangle+\sum_{\beta} c_{\beta}(t)|\beta\rangle \\
& =\sum_{\alpha} \psi_{\alpha}(t)|\alpha\rangle+\sum_{\beta} c_{\beta}(t)|\beta\rangle
\end{aligned}
$$

i.e.

$$
\psi_{\alpha}(t)=e^{-i \widehat{\mathcal{M}} t} \psi_{\alpha}(0)
$$

where

$$
\widehat{\mathcal{M}}=E_{0}+\mathcal{W} .
$$

$\widehat{\mathcal{M}}$, which governs the rate of decay of the amplitudes of the original states, is called the mass matrix. It is non-hermitian, but may be written in terms of two hermitian matrices $M$ and $\Gamma$ where

$$
\begin{aligned}
M & =\frac{1}{2}\left(\widehat{\mathcal{M}}+\widehat{\mathcal{M}}^{\dagger}\right) \\
\Gamma & =i\left(\widehat{\mathcal{M}}-\widehat{\mathcal{M}}^{\dagger}\right)
\end{aligned}
$$

such that

$$
\widehat{\mathcal{M}}=M-\frac{i}{2} \Gamma
$$

The elements of $M$ and $\Gamma$ are given by

$$
\begin{aligned}
M_{\alpha \alpha^{\prime}} & =E_{0} \delta_{\alpha \alpha^{\prime}}+\left\langle\alpha\left|\widehat{H^{\prime}}\right| \alpha^{\prime}\right\rangle+\sum_{\beta} \frac{\left\langle\alpha\left|\widehat{H^{\prime}}\right| \beta\right\rangle\left\langle\beta\left|\widehat{H^{\prime}}\right| \alpha^{\prime}\right\rangle}{\left(E_{0}-E_{\beta}\right)} \\
\Gamma_{\alpha \alpha^{\prime}} & =\sum_{\beta} 2 \pi \delta\left(E_{0}-E_{\beta}\right)\left\langle\alpha\left|\widehat{H^{\prime}}\right| \beta\right\rangle\left\langle\beta\left|\widehat{H^{\prime}}\right| \alpha^{\prime}\right\rangle .
\end{aligned}
$$

The coefficient $\psi(t)$ tells us how much of the state is left in undecayed at any given time $t$. If the eigenstates of $\widehat{\mathcal{M}}$, the so-called mass eigenstates, are known, then one may expand the operator $e^{-i \widehat{\mathcal{M}} t}$ easily, from whence the time development of the system may be calculated. It is important to remember, however, that $\psi(t)$ gives only the projection of the complete state $|\Psi(t)\rangle$ onto the space of the original states $|\alpha\rangle$. 


\section{Class diagrams}

The principles of object oriented design have been presented in chapter 3. In this appendix, the notation used to represent class diagrams, an essential tool in object oriented design, will be introduced, and the designs for the particle identification and global calibration schemes for the electromagnetic calorimeter represented in terms of these. Nomenclature of classes, in particular of their $\mathrm{C}++$ implementation, is widely used. For an explanation of terms, see any elementary $\mathrm{C}++$ text, such as Schildt[86]. For a more detailed explanation of the concepts and the language of $\mathrm{C}++$, see [87].

The most basic component of a class diagram is the class representation itself. A typical class diagram is shown in figure B-1. The name of the class is written at the top of the box. In the

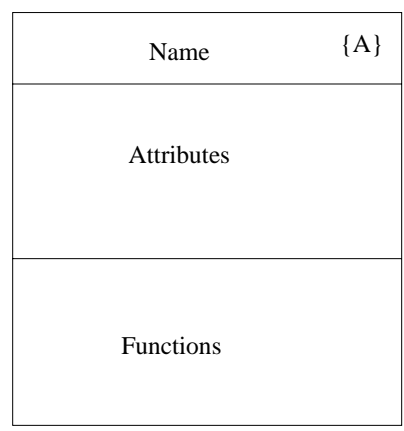

Figure B-1. The simplest representation of a class.

next box, some of the data members may be displayed. Since the implementation of other classes should not depend on this data, it is often omitted, and only included for information. The lower box is reserved for function names, which appear in the form

function: return type.

Not all functions need be shown, and in almost all cases, constructors, destructors and assignment operators are excluded.

Three key relationships between classes are represented by lines connecting the class boxes. These are shown in figure B-2. The top figure shows class A inheriting from class B. This means that class A can re-use all the public or protected methods (and data, if there are any) from class B, and 
re-implement any such functions that are virtual. If another function is given a pointer or reference to class B, that pointer or reference may actually refer to an instance of class $\mathrm{A}$, in which case the implementation of class A is used. The function need not know anything about class A, however. The $\{\mathrm{A}\}$ in the upper corner is used to denote an abstract class, in which at least one function is pure virtual, i.e. has no default implementation.
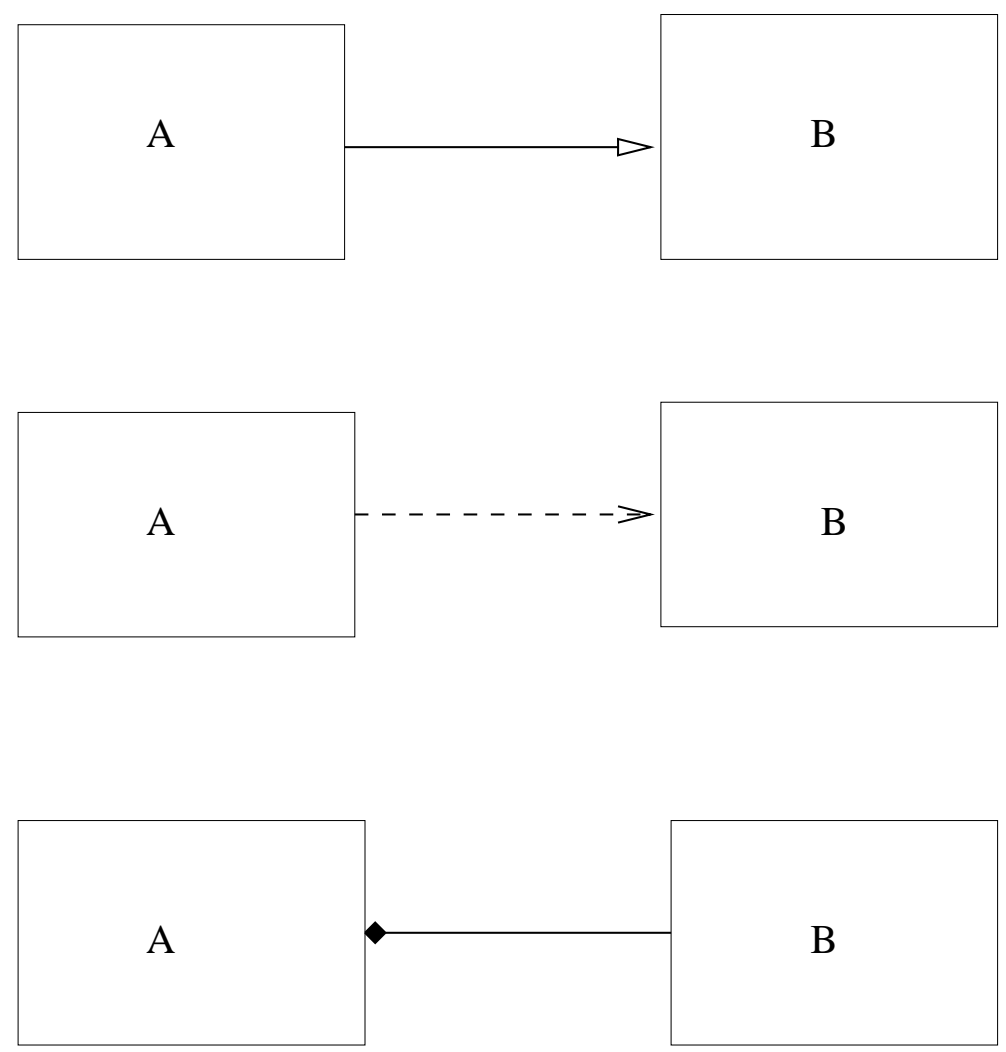

Figure B-2. Class relationships

The middle figure of the three represents a "uses" relationship-A "uses" B. In practice this means that class $\mathrm{A}$ has to know about the interface of class $\mathrm{B}$, and will make use of its public member functions.

The lower diagram represents a contains relationship. A contains (an instance of) B. The solid square indicates that $\mathrm{A}$ is responsible for the instance of $\mathrm{B}$, i.e. A owns $\mathrm{B}$. An alternative relationship, where A is not responsible for the instance of B (A contains B by reference or unowned pointer), a white square is used. 


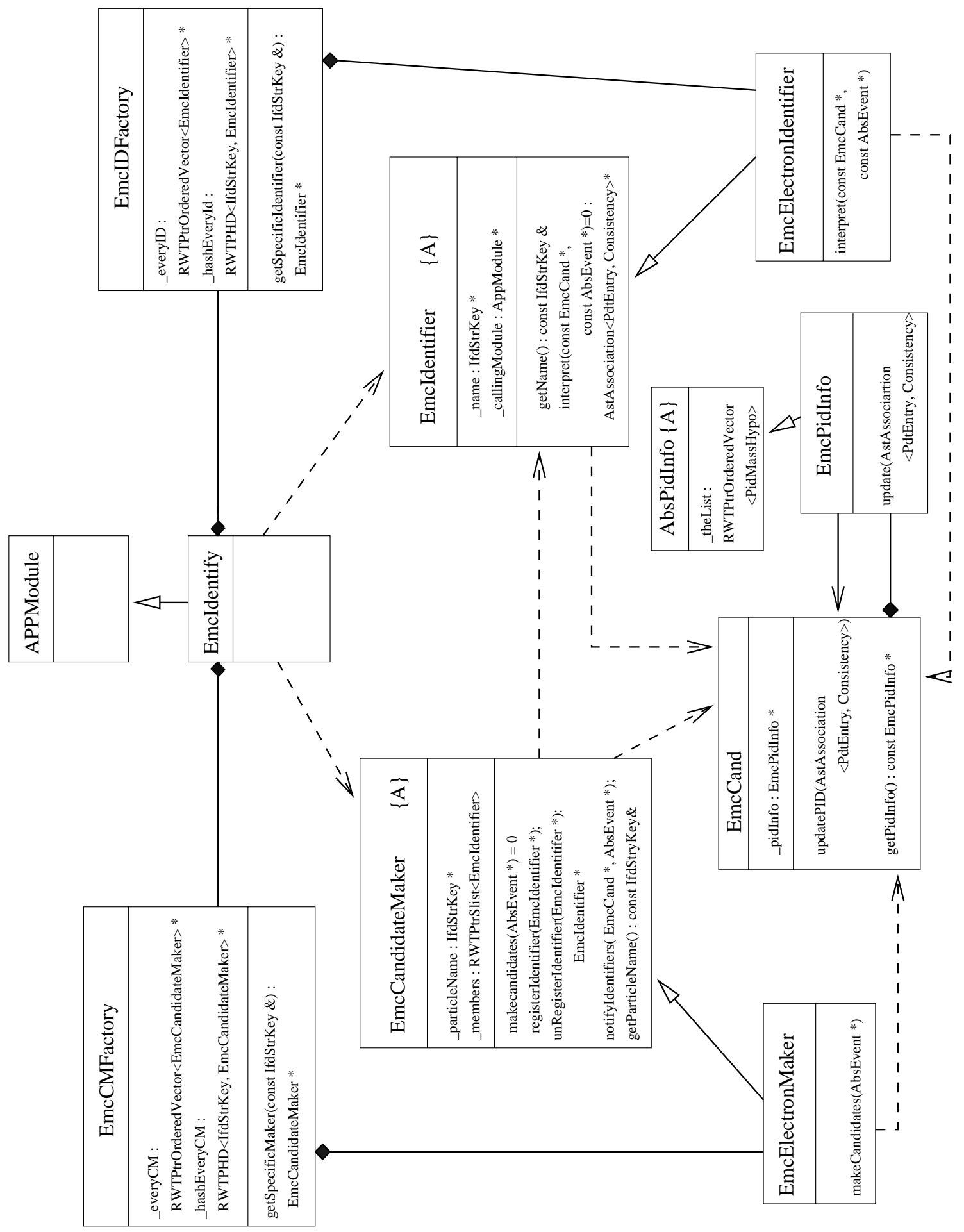

Figure B-3. Class diagram for the particle ID framework for the calorimeter. It is discussed more fully in chapter 3. 


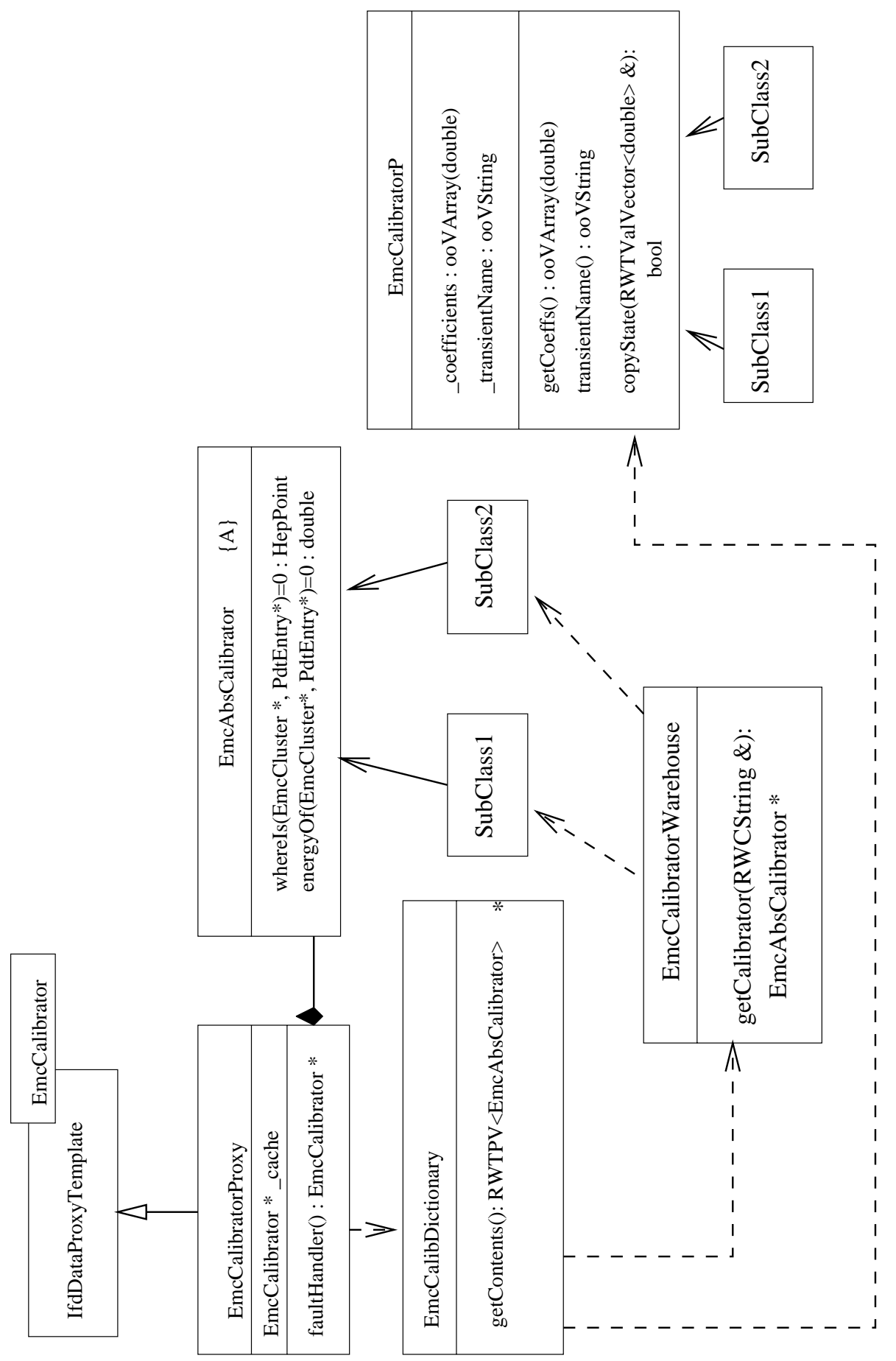

Figure B-4. Class diagram for the offline, global calibration system. This diagram refers to the retrieval side of the system. It is discussed more fully in chapter 3. 


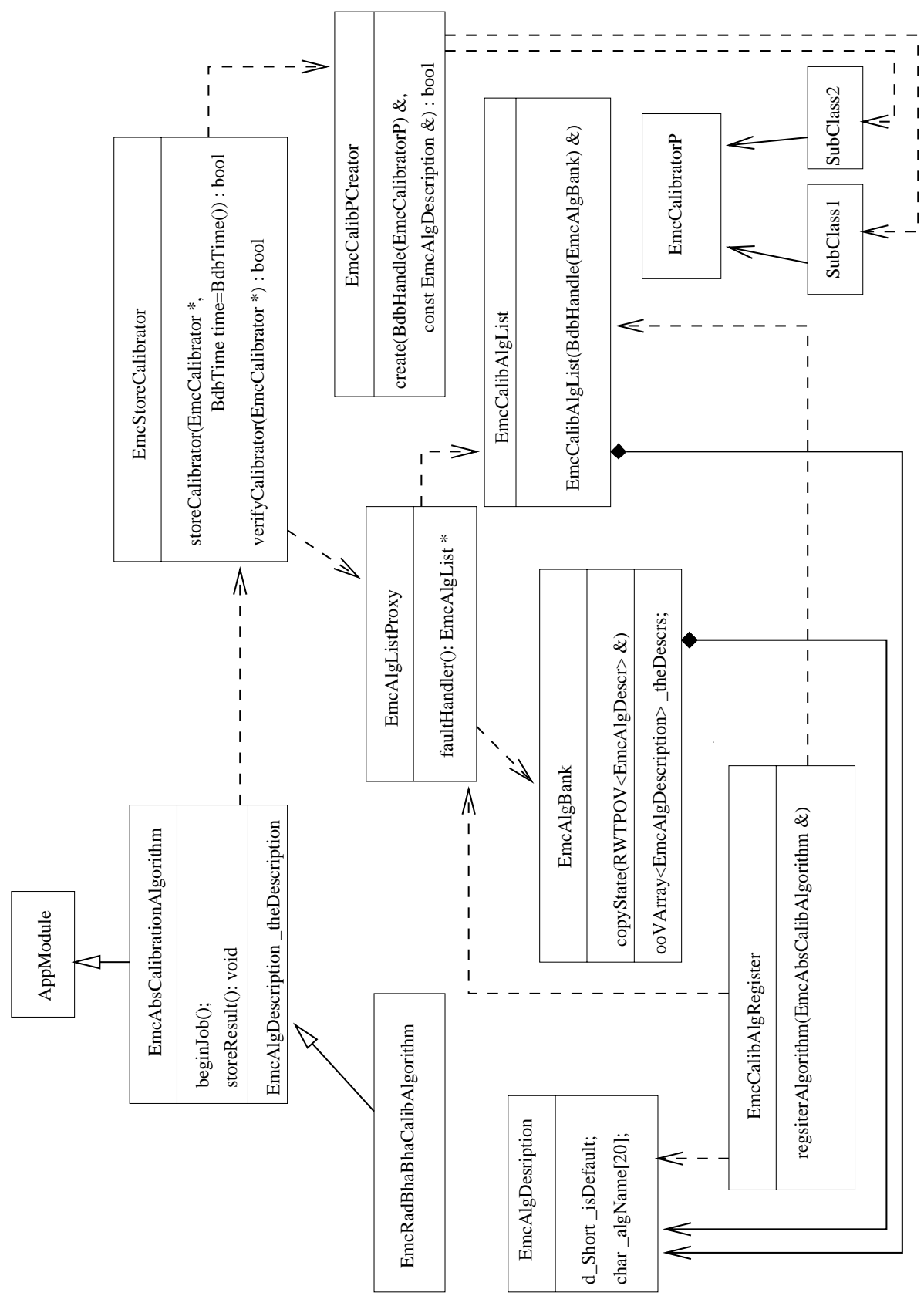

Figure B-5. Class diagram for the offline, global calibration system. This diagram refers to the storage side of the system. It is discussed more fully in chapter 3. 